\title{
Amorphous Solid Simulation and Trial Fabrication of the Organic Field-Effect Transistor of Tetrathienonaphthalenes Prepared by Using Microflow Photochemical Reactions; A Theoretical Calculation-Inspired Investigation
}

Atsushi Yamamoto, Yasunori Matsui, Toshio Asada, Motoki Kumeda, Kenichiro Takagi, Yu Suenaga, Kunihiko Nagae, Eisuke Ohta, Hiroyasu Sato, Shiro Koseki, Hiroyoshi Naito, and Hiroshi Ikeda*

Table of Contents

1. Amorphous Solid Structure Simulation $\quad$ S1

$\begin{array}{ll}\text { 2. Microflow Photochemical Reactor } & \text { S2 }\end{array}$

3. X-Ray Crystallographic Data $\quad$ S2

$\begin{array}{ll}\text { 4. Physical Properties } & \text { S3 }\end{array}$

$\begin{array}{lr}\text { 5. NMR Spectra } & \text { S7 }\end{array}$

$\begin{array}{lr}\text { 6. The Details of DFT Calculations } & \text { S18 }\end{array}$

$\begin{array}{ll}\text { 7. TD-DFT Output } & \text { S28 }\end{array}$

$\begin{array}{lr}\text { 8. References } & \text { S30 }\end{array}$

1. Amorphous Solid Structure Simulation
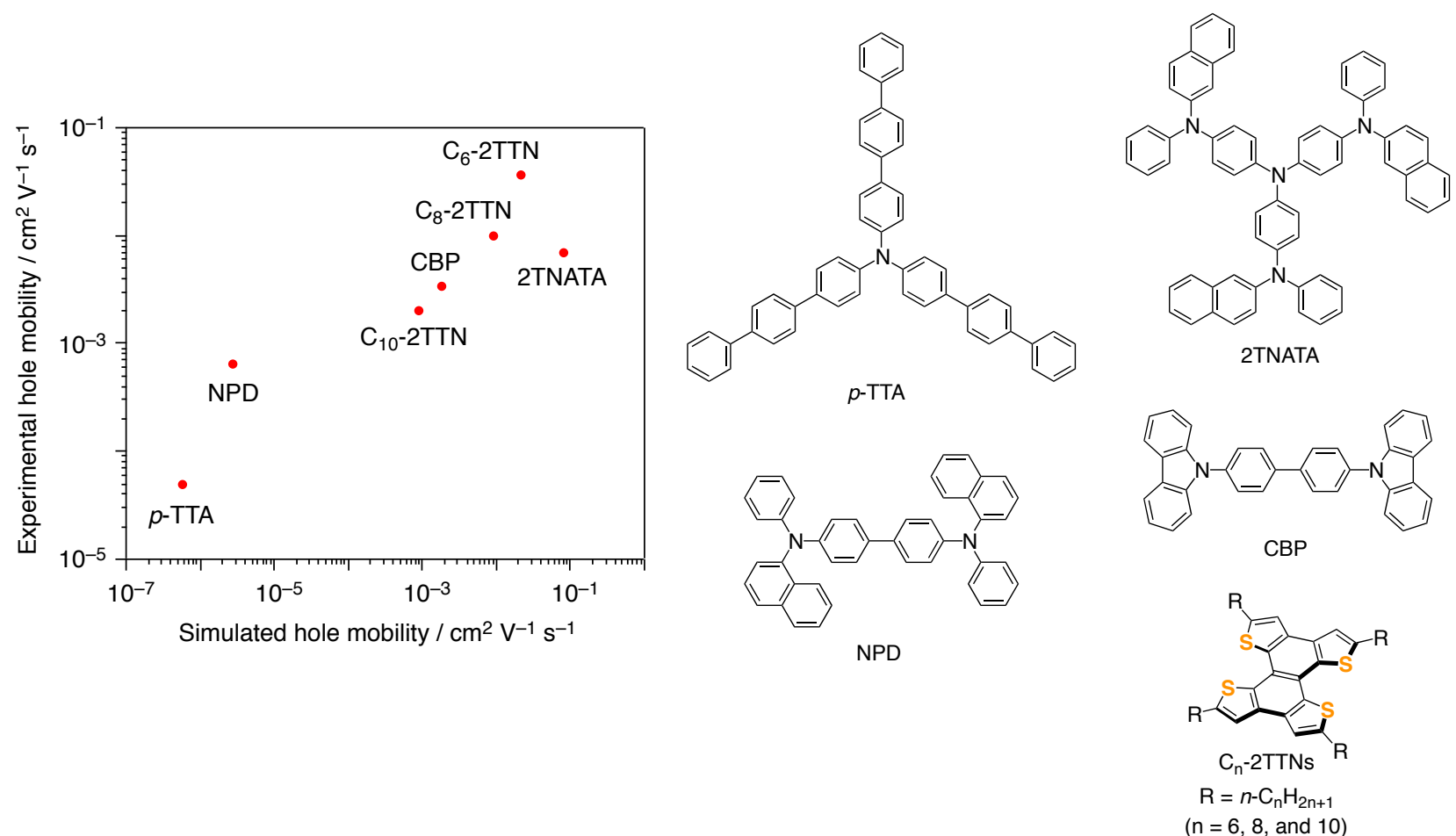

Fig. S1. Correlation between simulated and experimental hole mobilities of 4,4',4"-tris( $N$-2-naphthyl- $N$-phenylamino)triphenylamine (2TNATA), $\quad N, N^{\prime}$-di(1-naphthyl)- $N, N^{\prime}$-diphenylbenzidine $\quad$ (NPD), $\quad 4,4^{\prime}$-bis(carbazol-9-yl)biphenyl $\quad$ (CBP), 4,4',4"-tris(4-phenylphenyl)triphenylamine ( $p$-TTA), and $\mathrm{C}_{\mathrm{n}}$-2TTNs. 
(a)

(b)
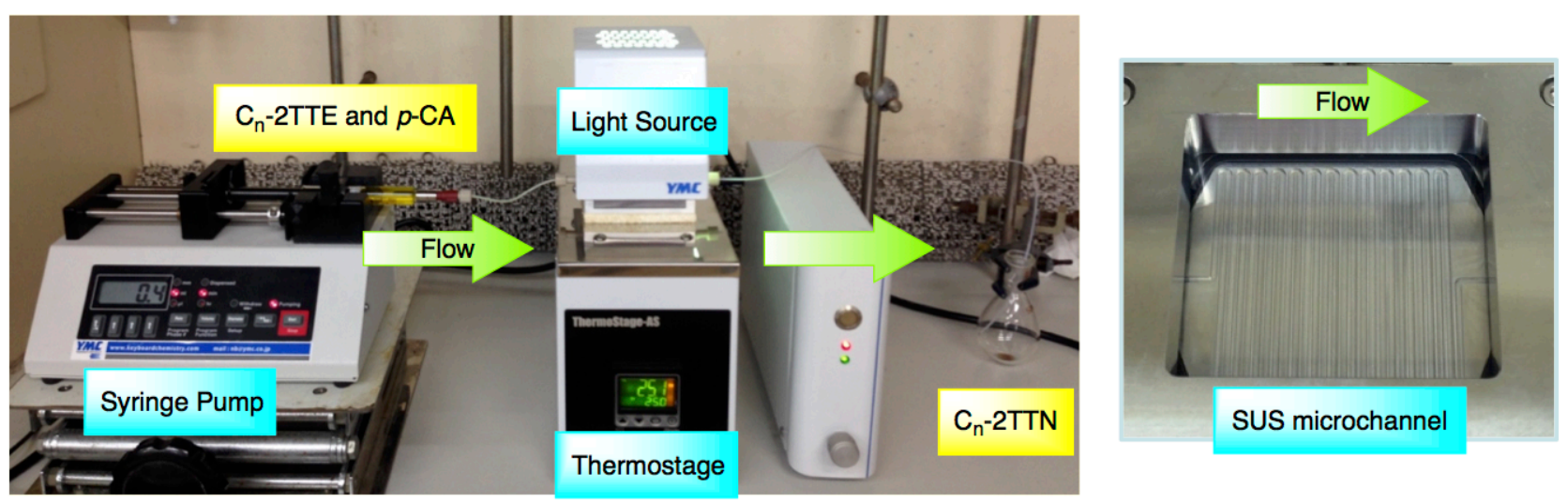

Fig. S2. Microflow reactor used to synthesis of $\mathrm{C}_{\mathrm{n}}-2 \mathrm{TTNs}$.

\section{X-Ray Crystallographic Data}

X-ray crystal structural analysis of 2 TTN were carried out on a Rigaku R-AXIS RAPID diffractometer, and analyzed by a Rigaku CrystalStructure crystallographic software package.

2TTN. Crystal data for $\mathrm{C}_{18} \mathrm{H}_{8} \mathrm{~S}_{4}$ : colorless needle, $0.32 \times 0.05 \times 0.02 \mathrm{~mm}$, monoclinic, $P 2_{1} / n, a=5.24926(13), b=$ $14.3321(3), c=18.3411(4) \AA, b=90.107(6) \mathrm{deg}, V=1379.86(6) \AA^{3}, Z=4, \rho_{\text {calcd }}=1.697 \mathrm{~g} \mathrm{~cm}^{-3}, T=296 \mathrm{~K}, 2 \theta_{\max }=68$ deg, $\mathrm{Cu}-\mathrm{K} \alpha$ radiation, $\lambda=0.15419 \AA, \mu=0.623 \mathrm{~mm}^{-1}, 9602$ reflections measured, 2482 unique reflections, $R_{\text {int }}=$ $0.0352,199$ parameters, $R_{1}=0.0361(I>2 \sigma l), \mathrm{w} R_{2}=0.1140$ (all data), CCDC-1438707.

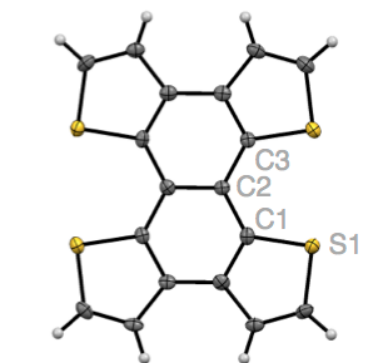

Front view

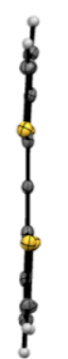

Side view

$$
\omega=\angle \mathrm{S} 1-\mathrm{C} 1-\mathrm{C} 2-\mathrm{C} 3=3.55 \mathrm{deg}
$$

Fig. S3. ORTEP plots of structural data of $2 \mathrm{TTN}$ obtained by using X-ray crystallographic analysis showing $50 \%$-probability thermal ellipsoids $(T=296 \mathrm{~K})$. 


\section{4-1. DSC Traces}
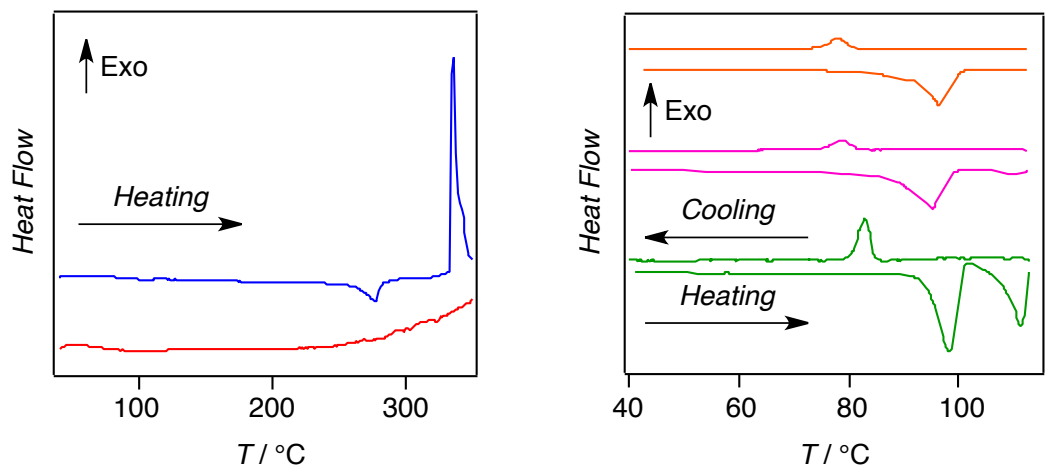

Fig. S4. DSC traces of of 3TTN (blue), 2 TTN (red), $\mathrm{C}_{6^{-}}$(orange), $\mathrm{C}_{8^{-}}$(pink), and $\mathrm{C}_{10^{-}}-2 \mathrm{TTNs}$ (green).

\section{4-2. Cyclic Voltammmograms}

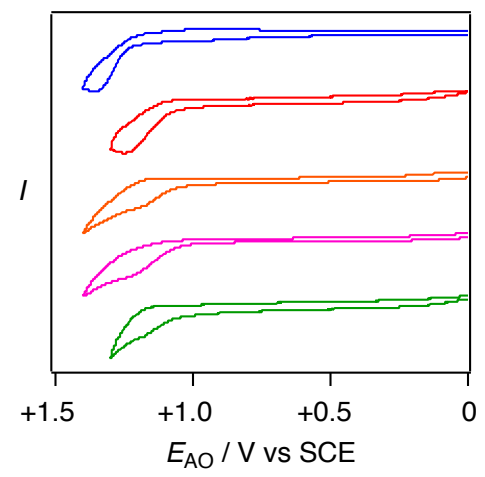

Fig. S5. Cyclic voltammmograms of $3 \mathrm{TTN}$ (blue), $2 \mathrm{TTN}$ (red), $\mathrm{C}_{6^{-}}$(orange), $\mathrm{C}_{8^{-}}$(pink), and $\mathrm{C}_{10^{-}} 2 \mathrm{TTNs}(\mathrm{green})$ in $\mathrm{CH}_{2} \mathrm{Cl}_{2}$ containing $0.1 \mathrm{M} \mathrm{n}-\mathrm{Bu}_{4} \mathrm{~N}^{+} \mathrm{BF}_{4}^{-}$. Scan rate: $0.1 \mathrm{~V} \mathrm{~s}^{-1}$, reference electrode: SCE, working and counter electrodes: Pt.

\section{4-3. UV-Vis Absorption Spectra and Electronic Transitions}

(a)

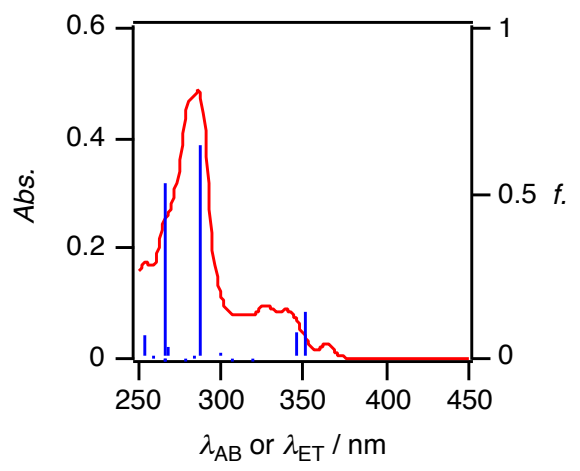

(b)

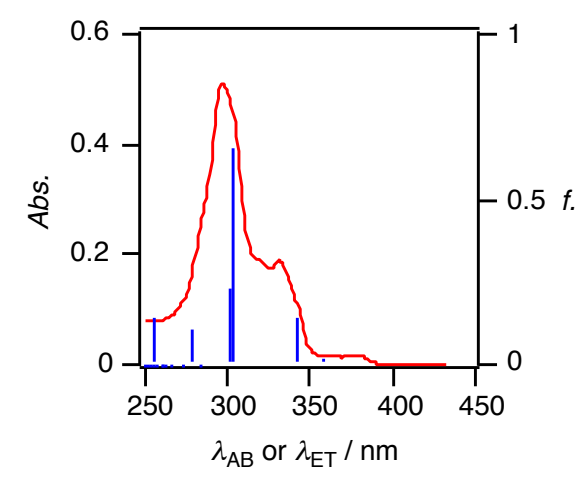

Fig. S6. UV-Vis absorption (red) spectra and electronic transitions [blue, TD-B3LYP/6-311+G(d,p)] of (a) $3 T T N$ and (b) 2 TTN (1.0 $\times 10^{-5} \mathrm{M}$, respectively) in aerated $\mathrm{CH}_{2} \mathrm{Cl}_{2}$. 


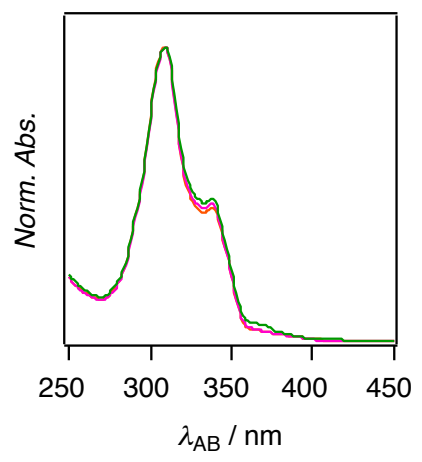

Fig. S7. UV-Vis absorption spectra of $\mathrm{C}_{6^{-}}$(orange), $\mathrm{C}_{8^{-}}$(pink), and $\mathrm{C}_{10^{-}}-2 \mathrm{TTNs}$ (green, $1.0 \times 10^{-5} \mathrm{M}$, respectively) in aerated $\mathrm{CH}_{2} \mathrm{Cl}_{2}$.

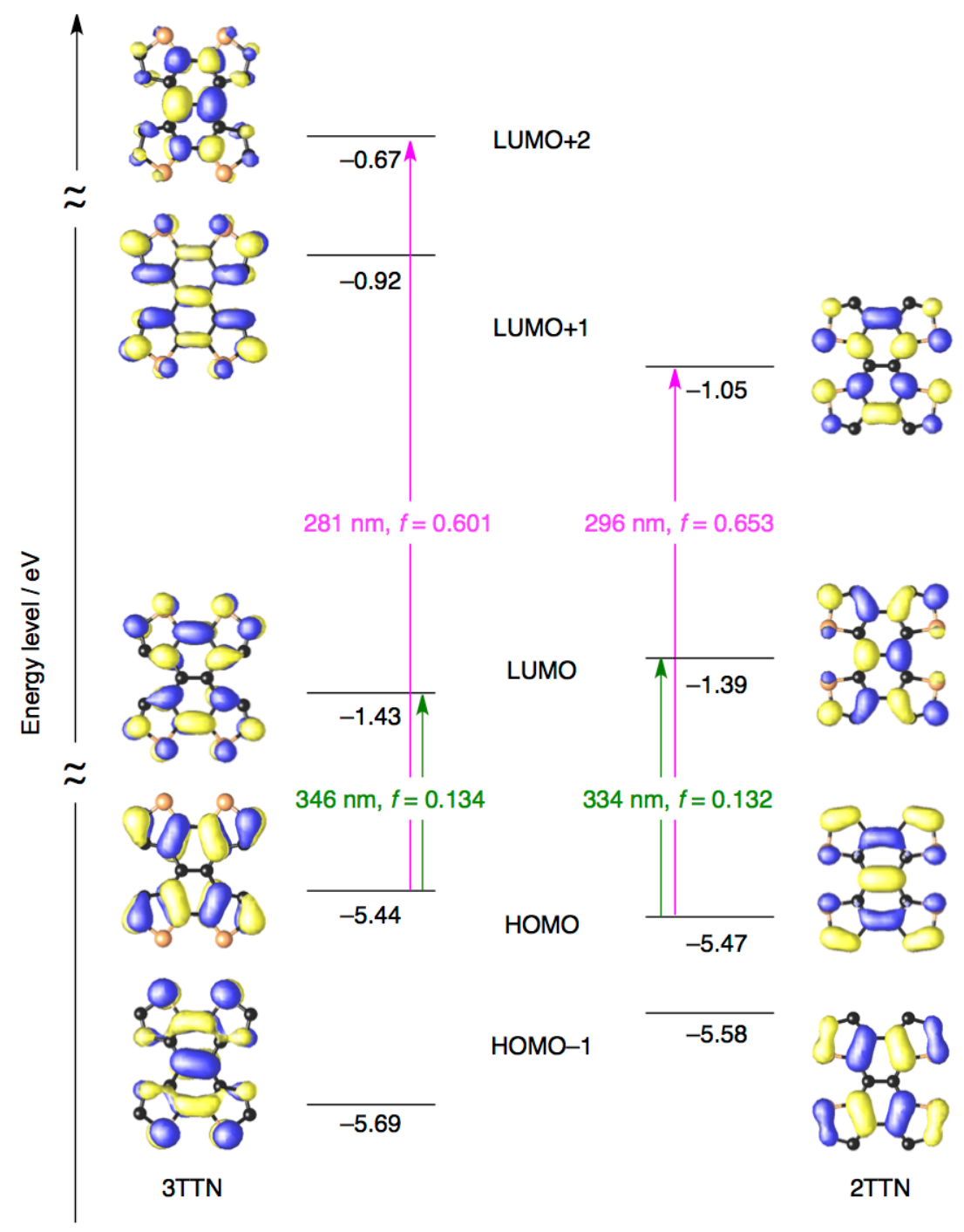

Fig. S8. The energy level of HOMO-1, HOMO, LUMO, LUMO+1, and LUMO+2 of 3TTN and 2TTN calculated with TD-DFT method [B3LYP/6-311+G(d,p)]. 
(a)

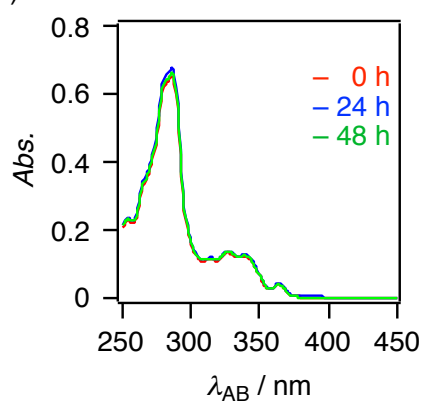

(b)

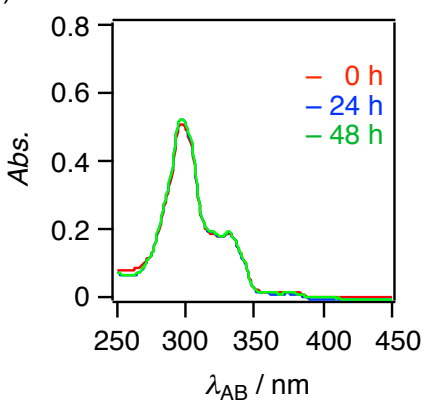

Fig. S9. Time course of the UV-Vis absorption spectra of (a) $3 \mathrm{TTN}$ and (b) $2 \mathrm{TTN}\left(1 \times 10^{-5} \mathrm{M}\right.$, resectively) in aerated $\mathrm{CH}_{2} \mathrm{Cl}_{2}$ for 48 h.

\section{4-4. Polarizing Micrographs of Thin Film}

(a)

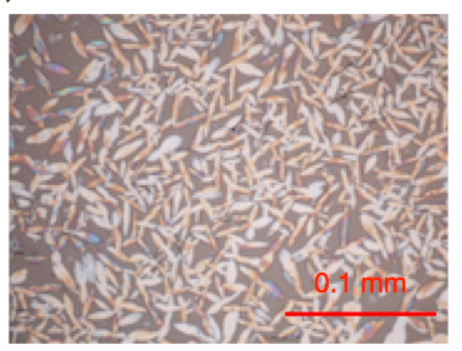

(b)

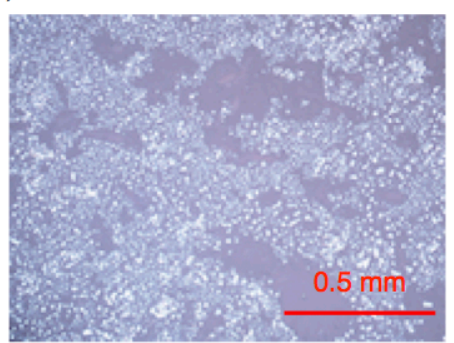

(c)

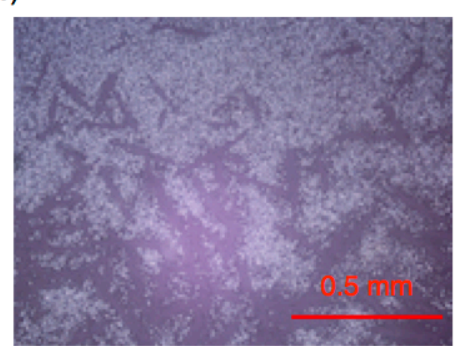

Fig. S10. Polarizing micrographs of annealed thin films of (a) $\mathrm{C}_{6^{-}}$, (b) $\mathrm{C}_{8^{-}}$, and (c) $\mathrm{C}_{10^{-}}-2 \mathrm{TTNs}$ at $c a .100{ }^{\circ} \mathrm{C}$ after spincoating. 
(a)
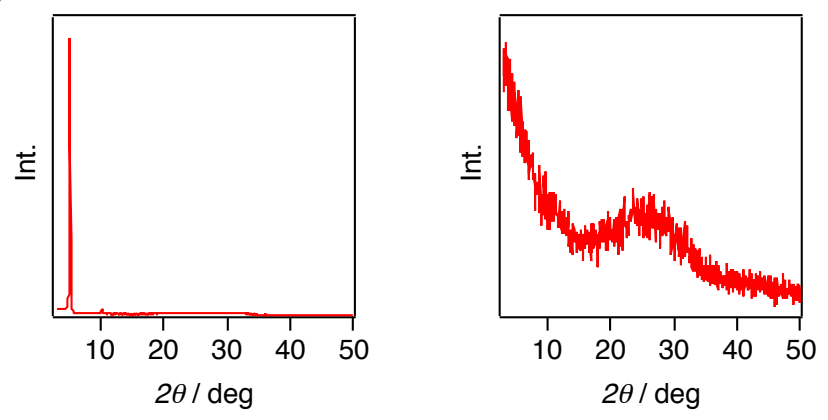

(b)
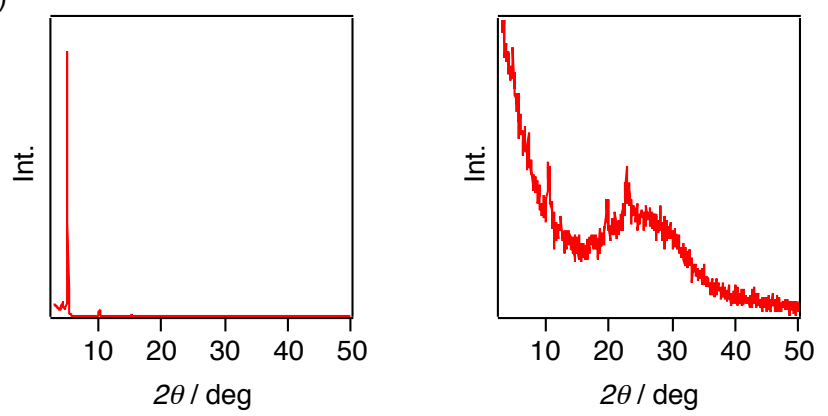

Fig. S11. XRD patterns (left, out-of-plane measurements and right, in-plane measurements) of (a) $\mathrm{C}_{8^{-}}$and (b) $\mathrm{C}_{10^{-}}-2 \mathrm{TTN}$.

\section{4-6. OFET Characteristics}

(a)
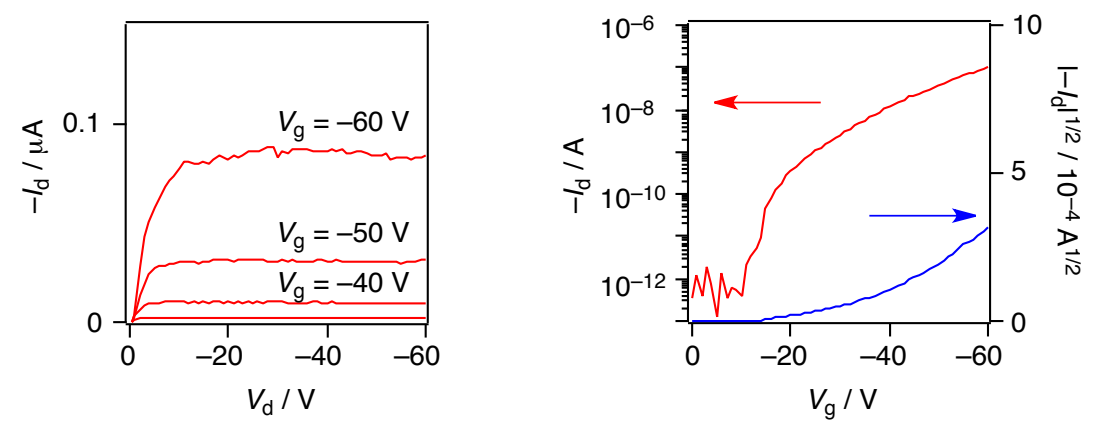

(b)
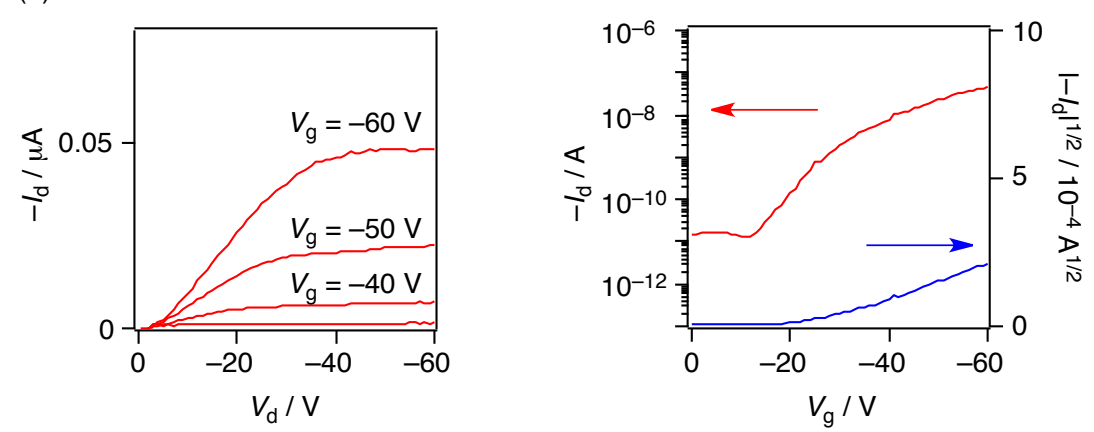

Fig. S12. OFET characteristics (left, output characteristics, and right, transfer characteristics in the saturation region at drain voltage of $-60 \mathrm{~V}$ ) of (a) $\mathrm{C}_{8^{-}}$and (b) $\mathrm{C}_{10}$-2TTNs. The spincoated thin films were prepared from their toluene solution (1 wt\%) at room temperature. 
5. NMR Spectra

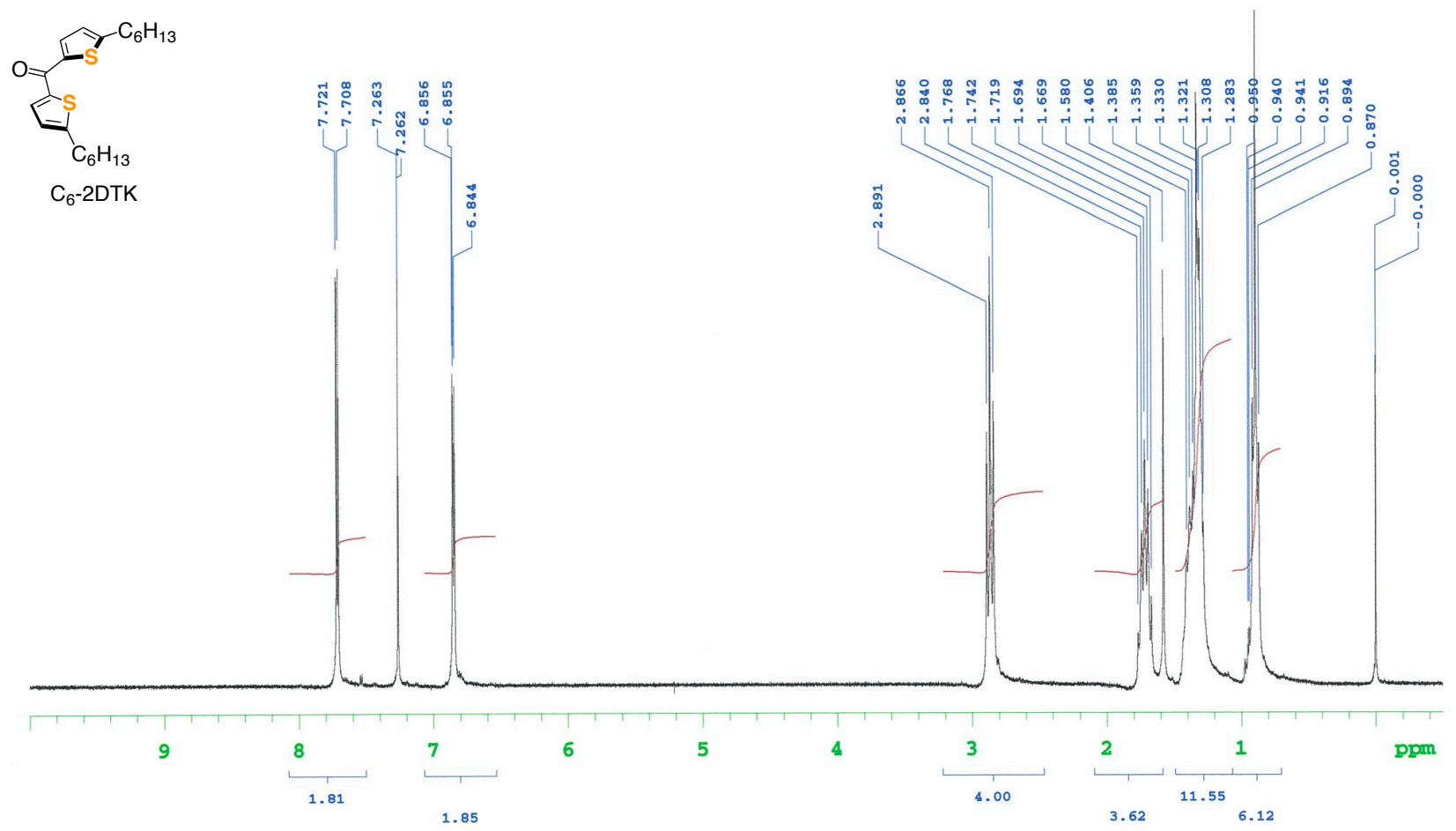

Fig. S13. ${ }^{1} \mathrm{H}$ NMR spectrum of $\mathrm{C}_{6}-2 \mathrm{DTK}$ in $\mathrm{CDCl}_{3}$.

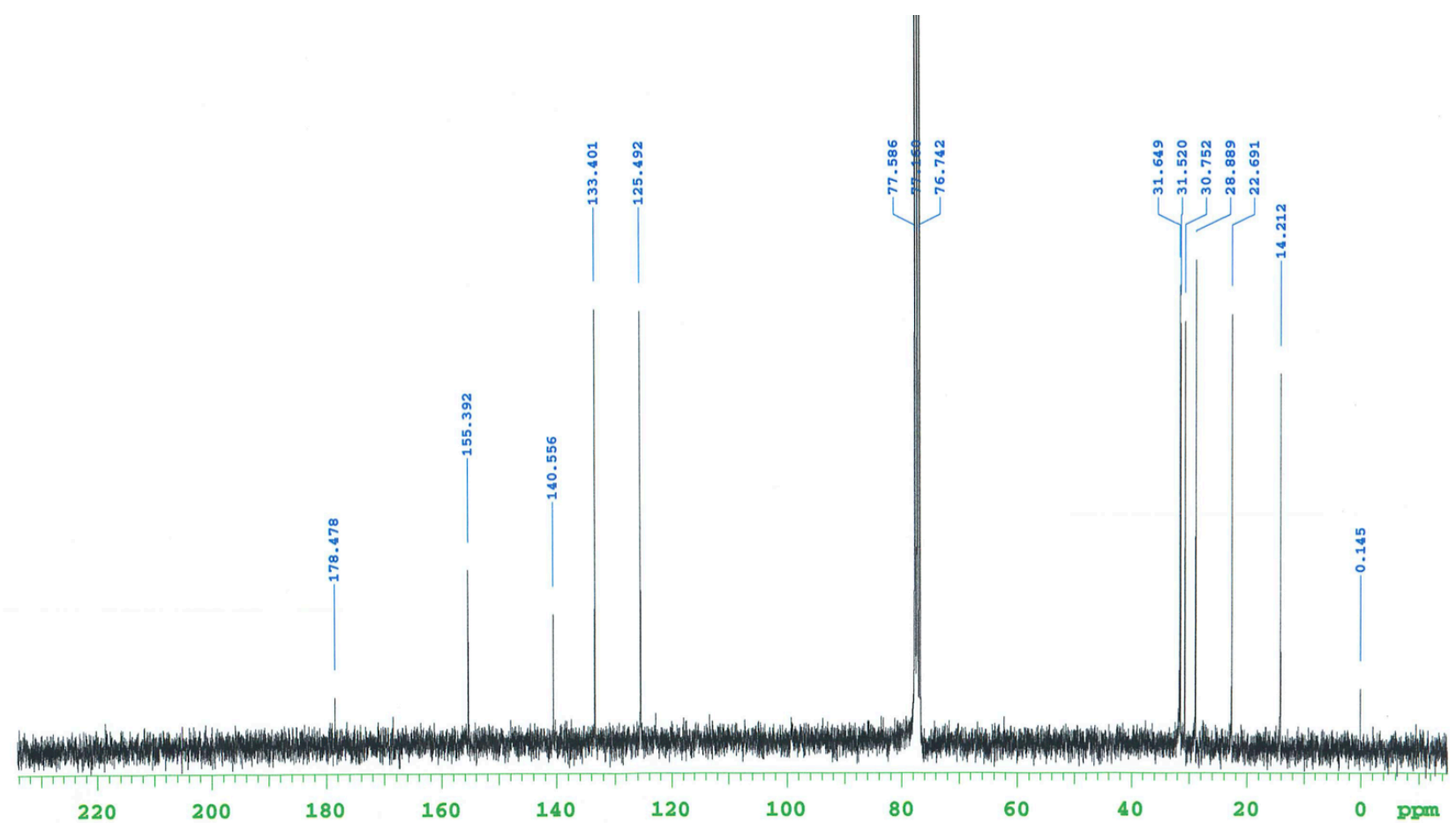

Fig. S14. ${ }^{13} \mathrm{C}$ NMR spectrum of $\mathrm{C}_{6}$-2DTK in $\mathrm{CDCl}_{3}$. 

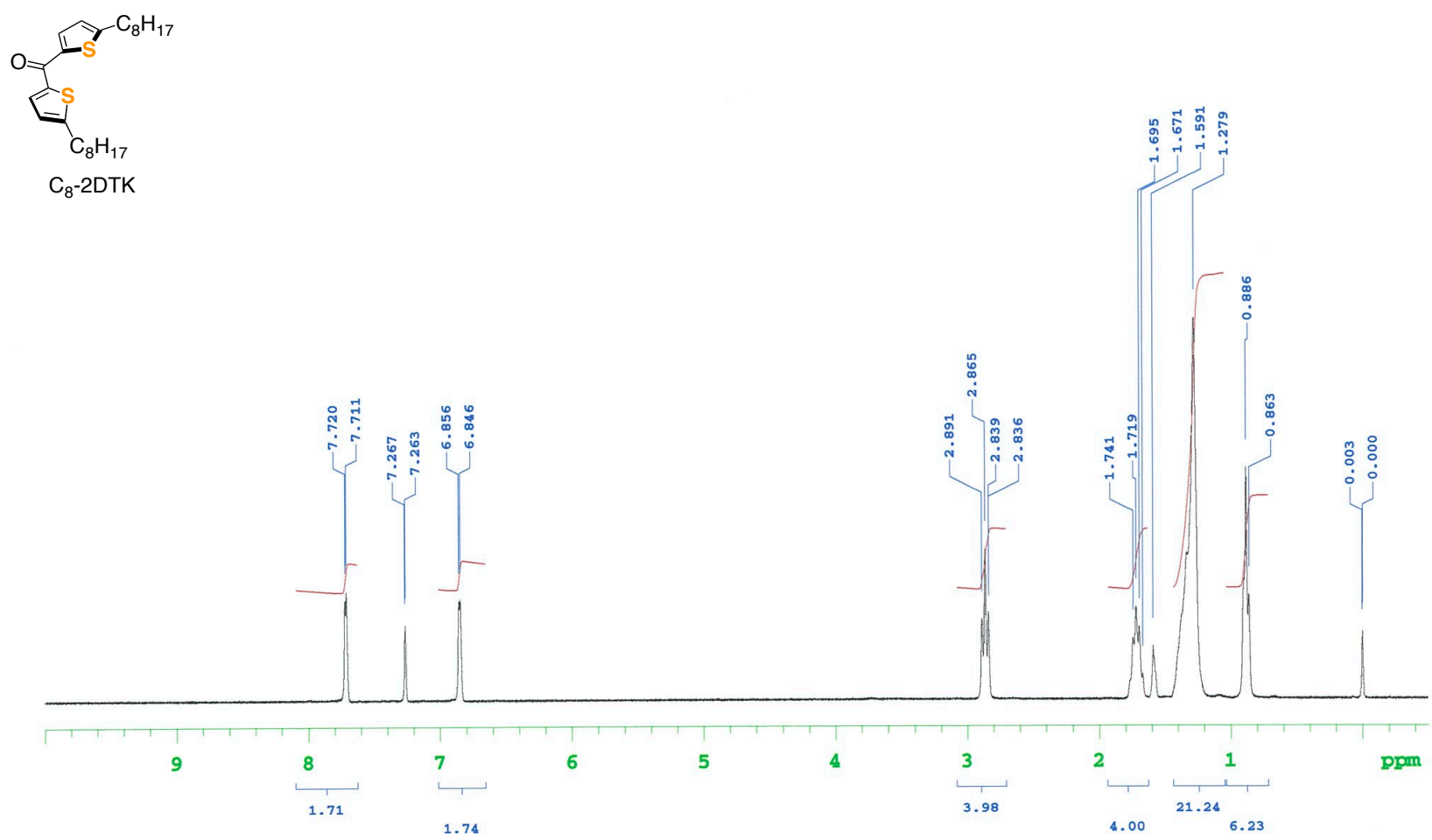

Fig. S15. ${ }^{1} \mathrm{H}$ NMR spectrum of $\mathrm{C}_{8}-2 \mathrm{DTK}$ in $\mathrm{CDCl}_{3}$.

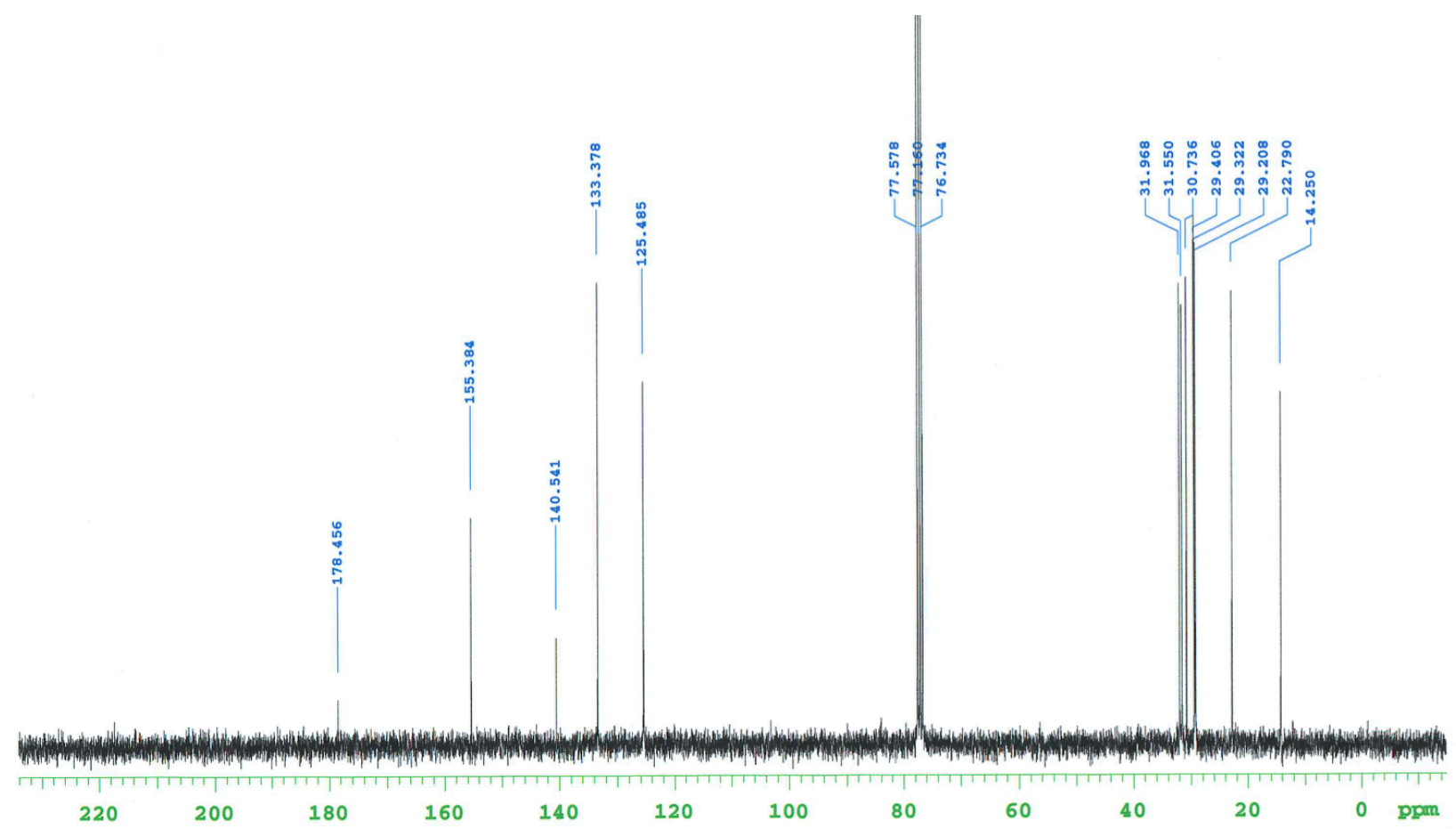

Fig. S16. ${ }^{13} \mathrm{C}$ NMR spectrum of $\mathrm{C}_{8}-2 \mathrm{DTK}$ in $\mathrm{CDCl}_{3}$. 

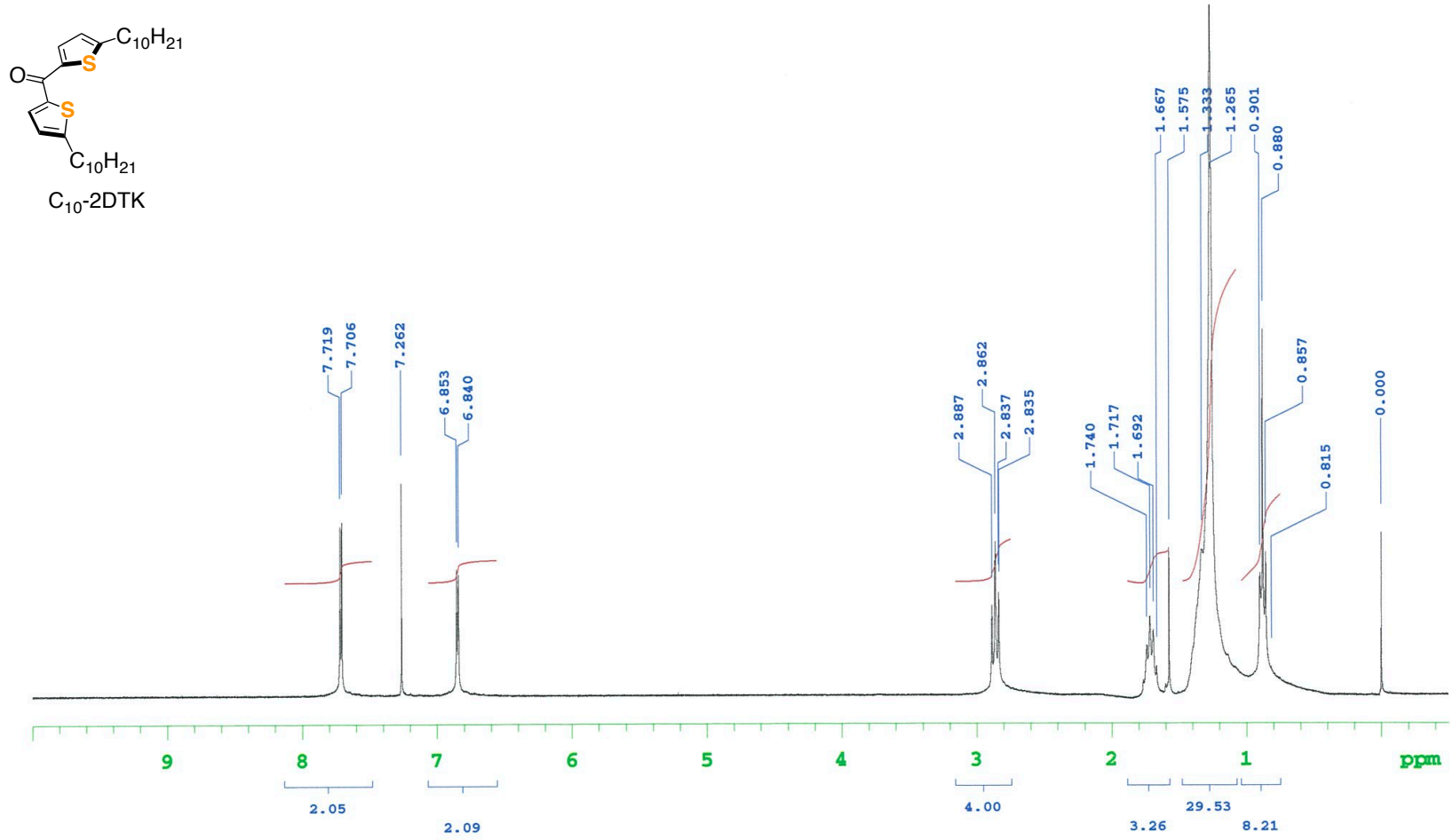

Fig. S17. ${ }^{1} \mathrm{H}$ NMR spectrum of $\mathrm{C}_{10}-2 \mathrm{DTK}$ in $\mathrm{CDCl}_{3}$.

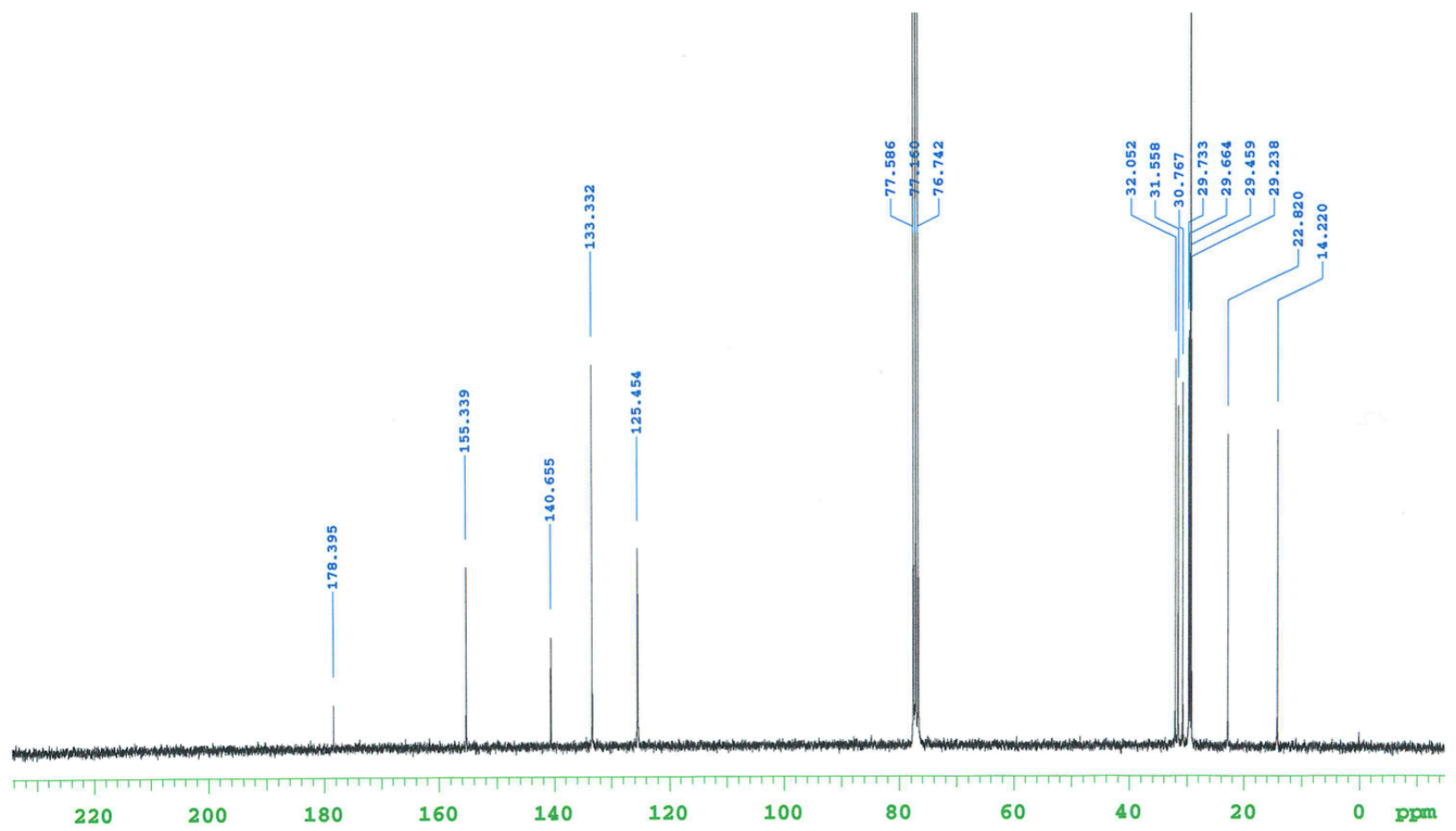

Fig. S18. ${ }^{13} \mathrm{C}$ NMR spectrum of $\mathrm{C}_{10}-2 \mathrm{DTK}$ in $\mathrm{CDCl}_{3}$. 


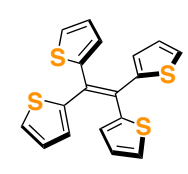

2TTE

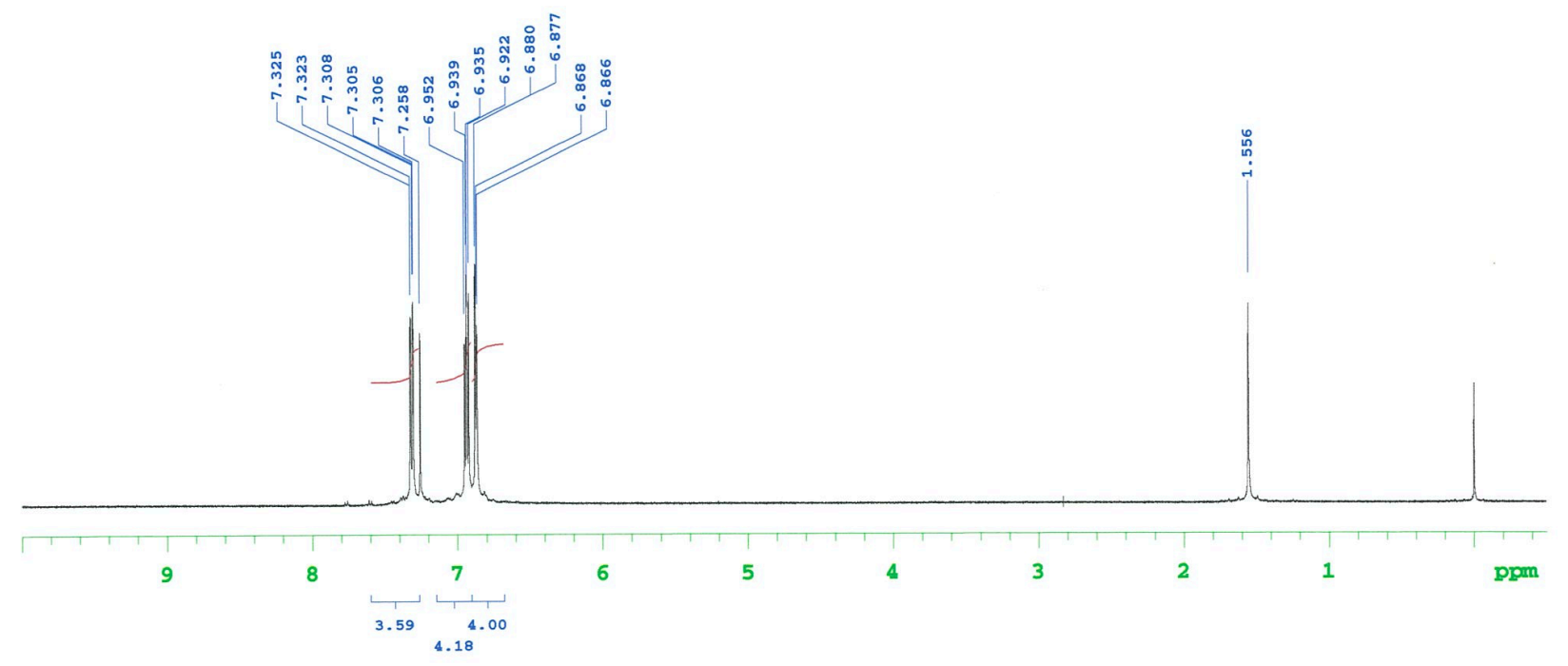

Fig. S19. ${ }^{1} \mathrm{H}$ NMR spectrum of $2 \mathrm{TTE}$ in $\mathrm{CDCl}_{3}$.

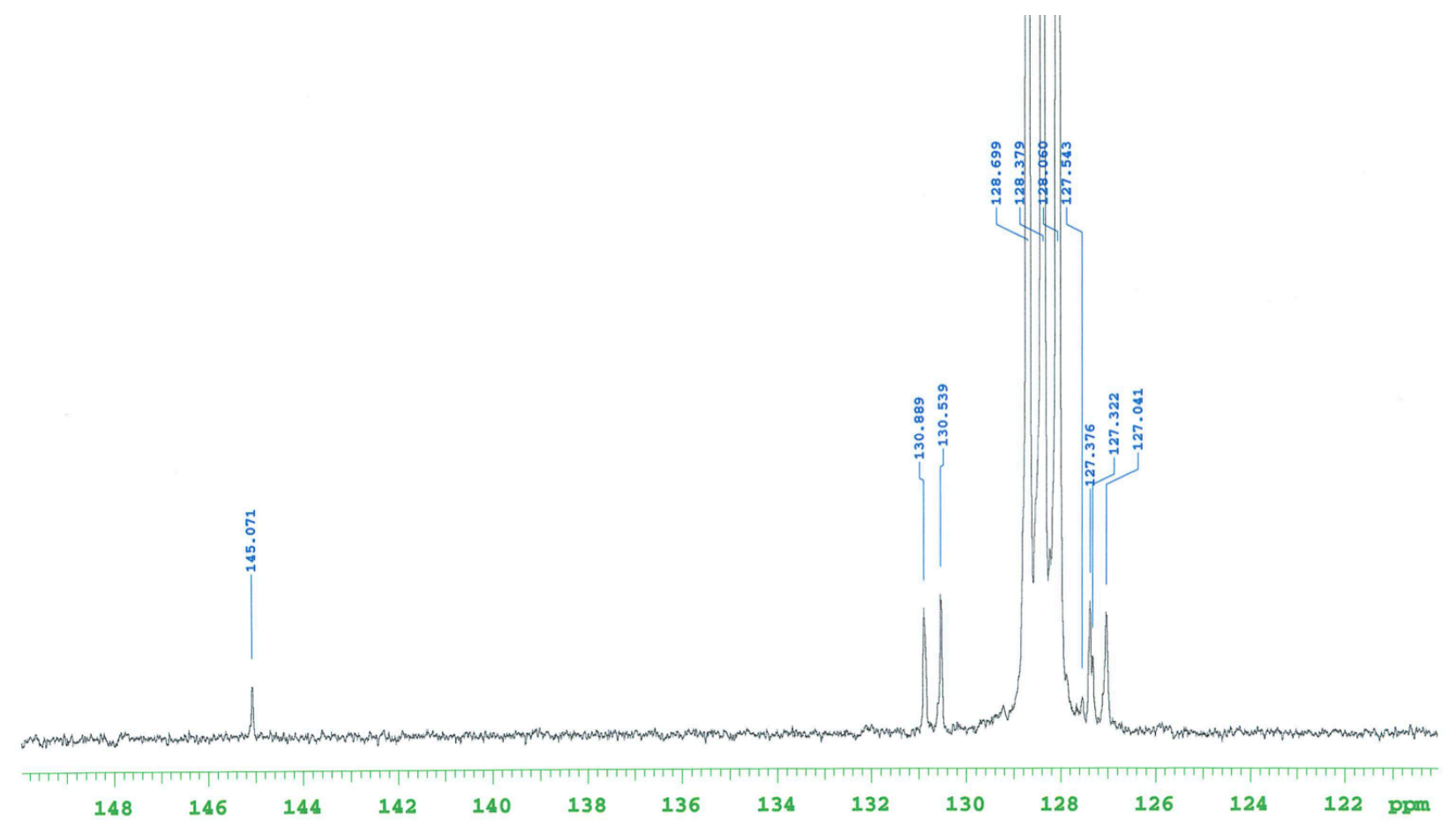

Fig. S20. ${ }^{13} \mathrm{C}$ NMR spectrum of $2 \mathrm{TTE}$ in $\mathrm{C}_{6} \mathrm{D}_{6}$. 


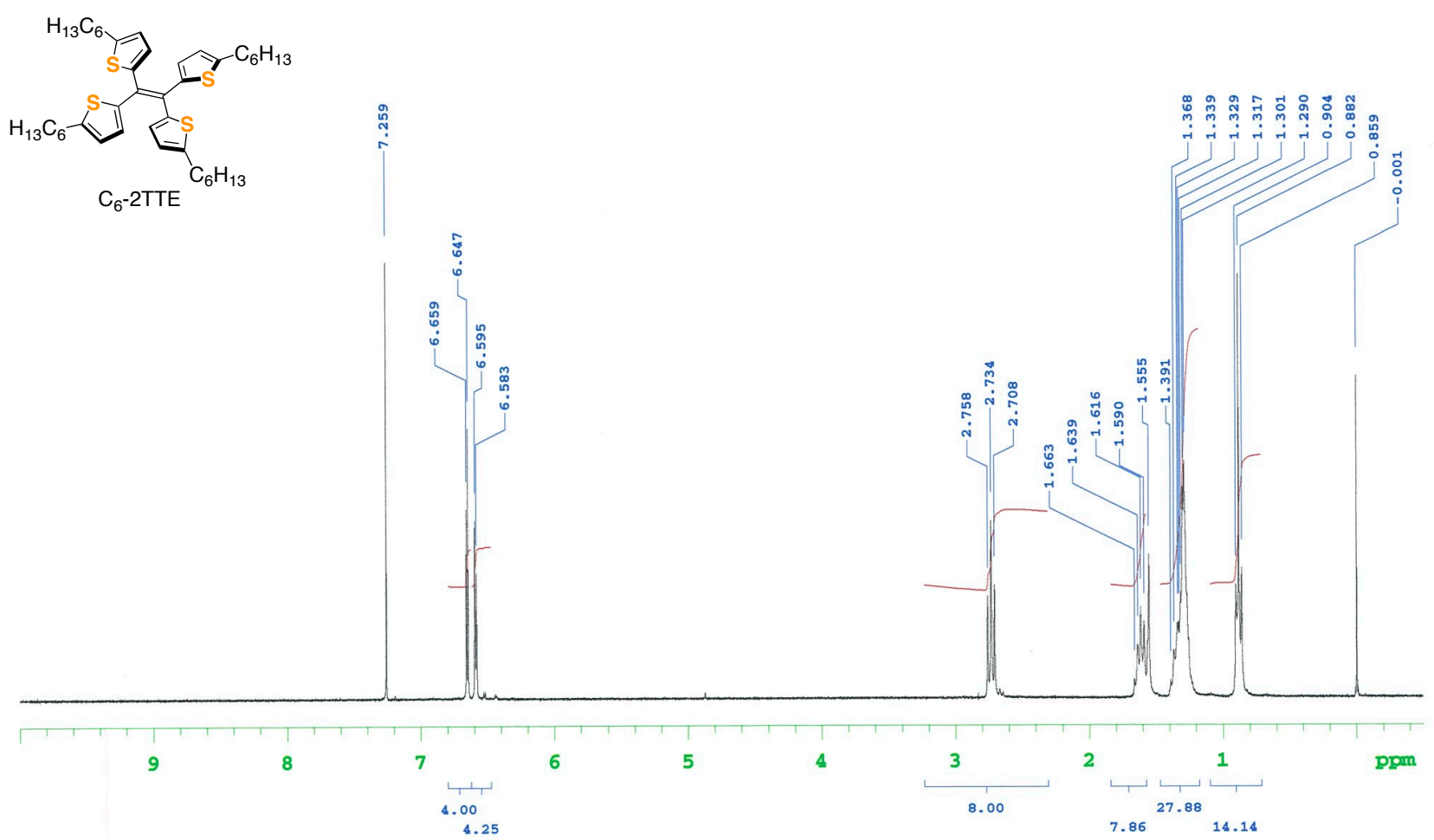

Fig. S21. ${ }^{1} \mathrm{H}$ NMR spectrum of $\mathrm{C}_{6}-2 \mathrm{TTE}$ in $\mathrm{CDCl}_{3}$.

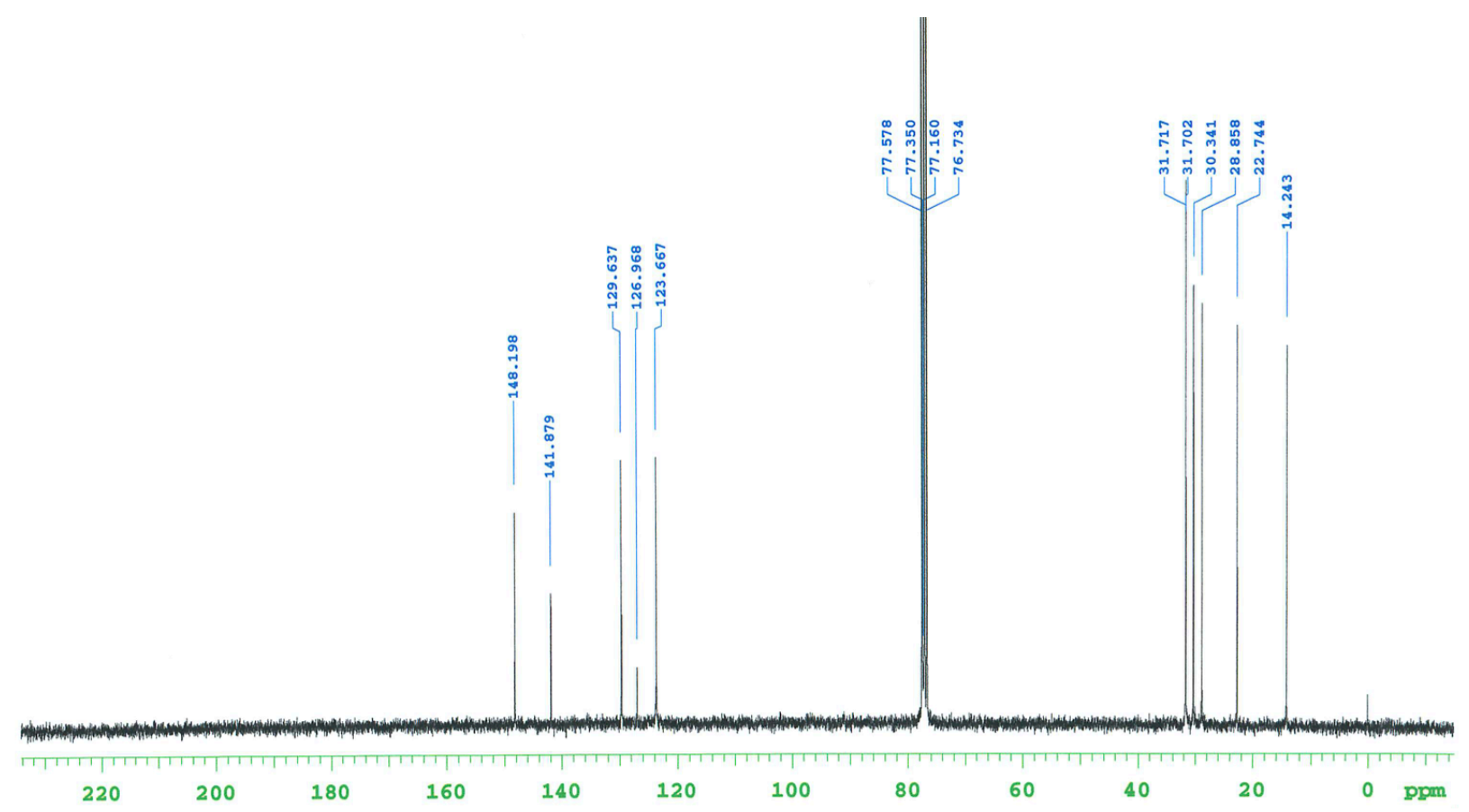

Fig. S22. ${ }^{13} \mathrm{C}$ NMR spectrum of $\mathrm{C}_{6}-2 \mathrm{TTE}$ in $\mathrm{CDCl}_{3}$. 


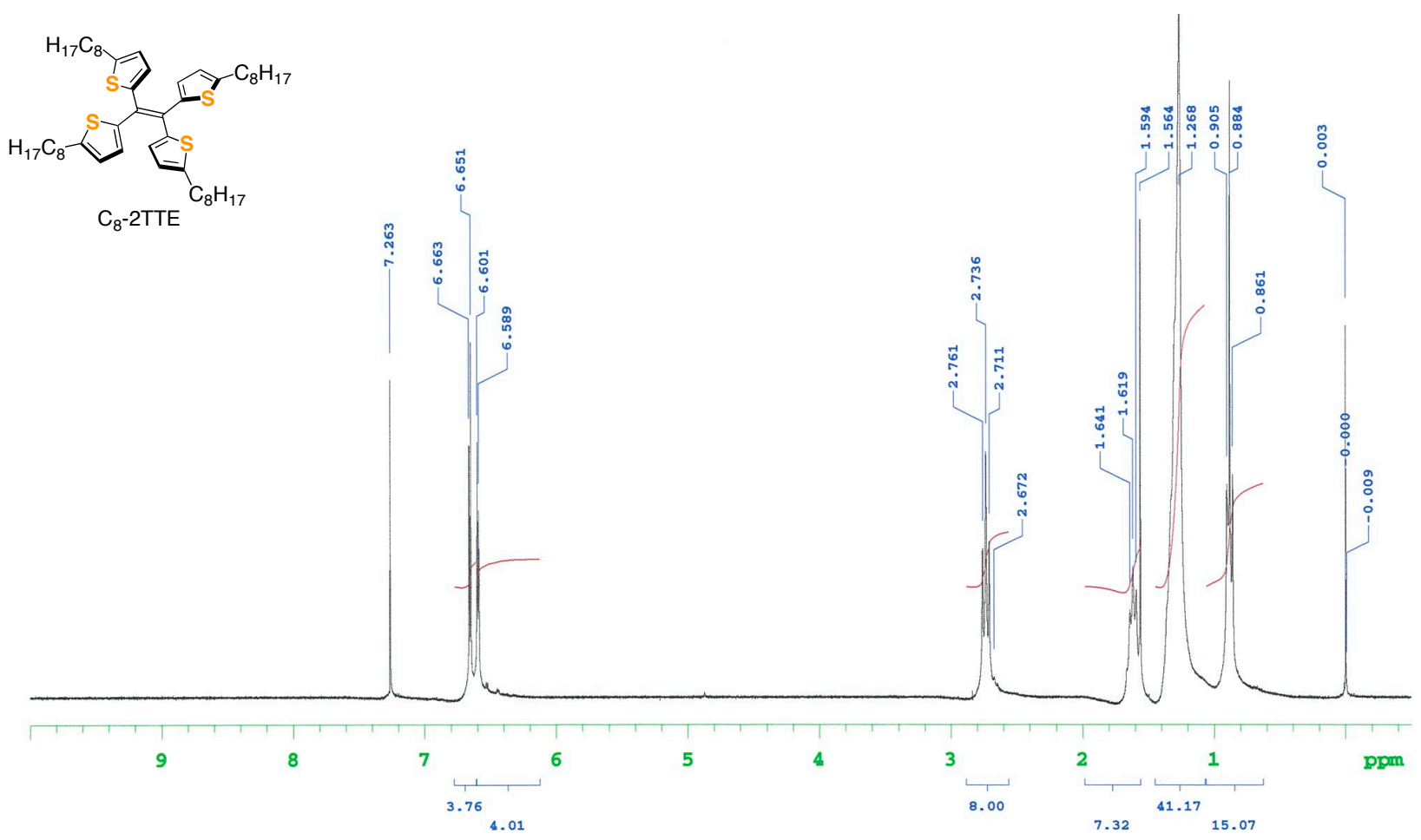

Fig. S23. ${ }^{1} \mathrm{H}$ NMR spectrum of $\mathrm{C}_{8}-2 \mathrm{TTE}$ in $\mathrm{CDCl}_{3}$.

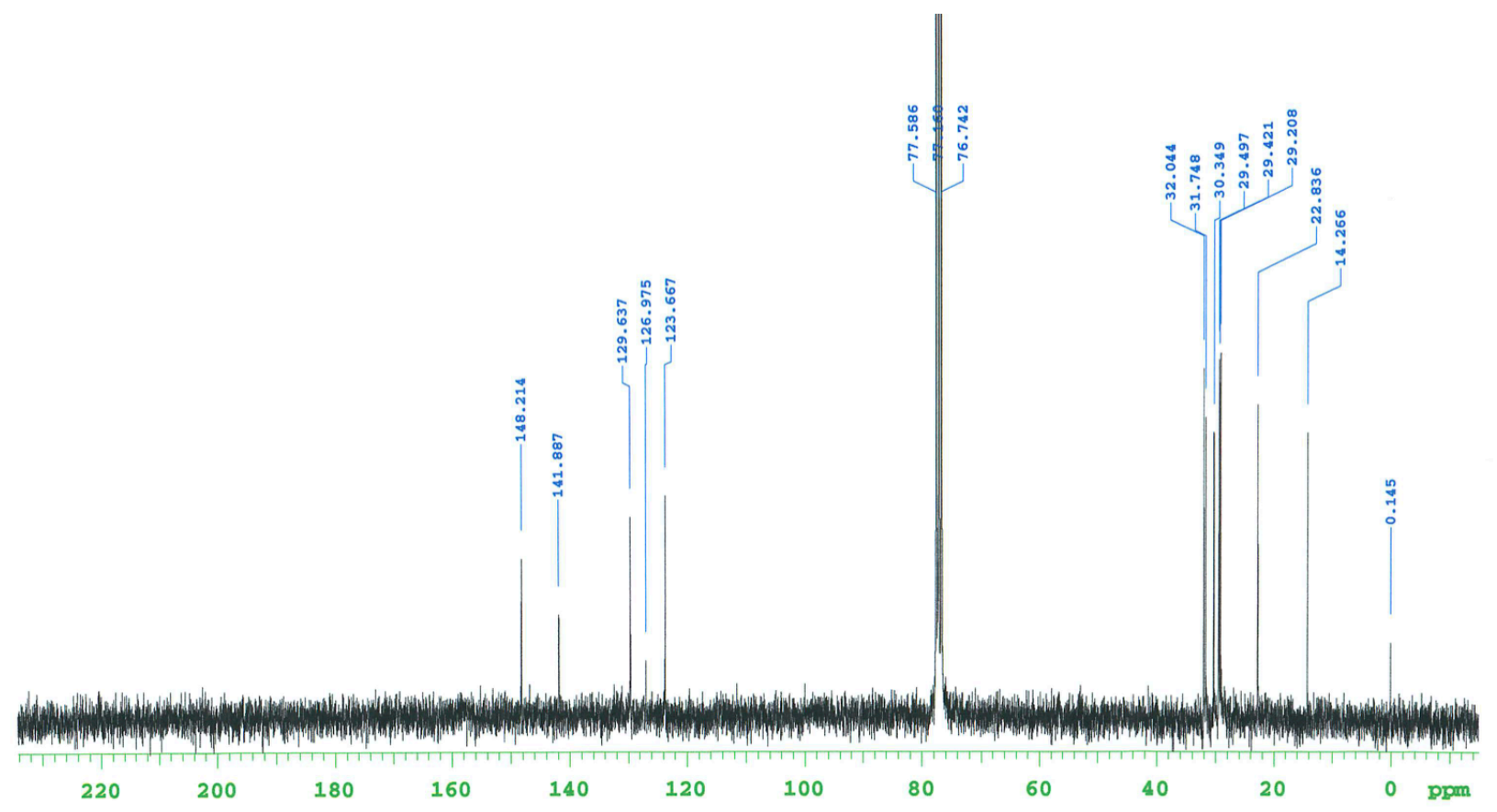

Fig. S24. ${ }^{13} \mathrm{C}$ NMR spectrum of $\mathrm{C}_{8}-2 \mathrm{TTE}$ in $\mathrm{CDCl}_{3}$. 

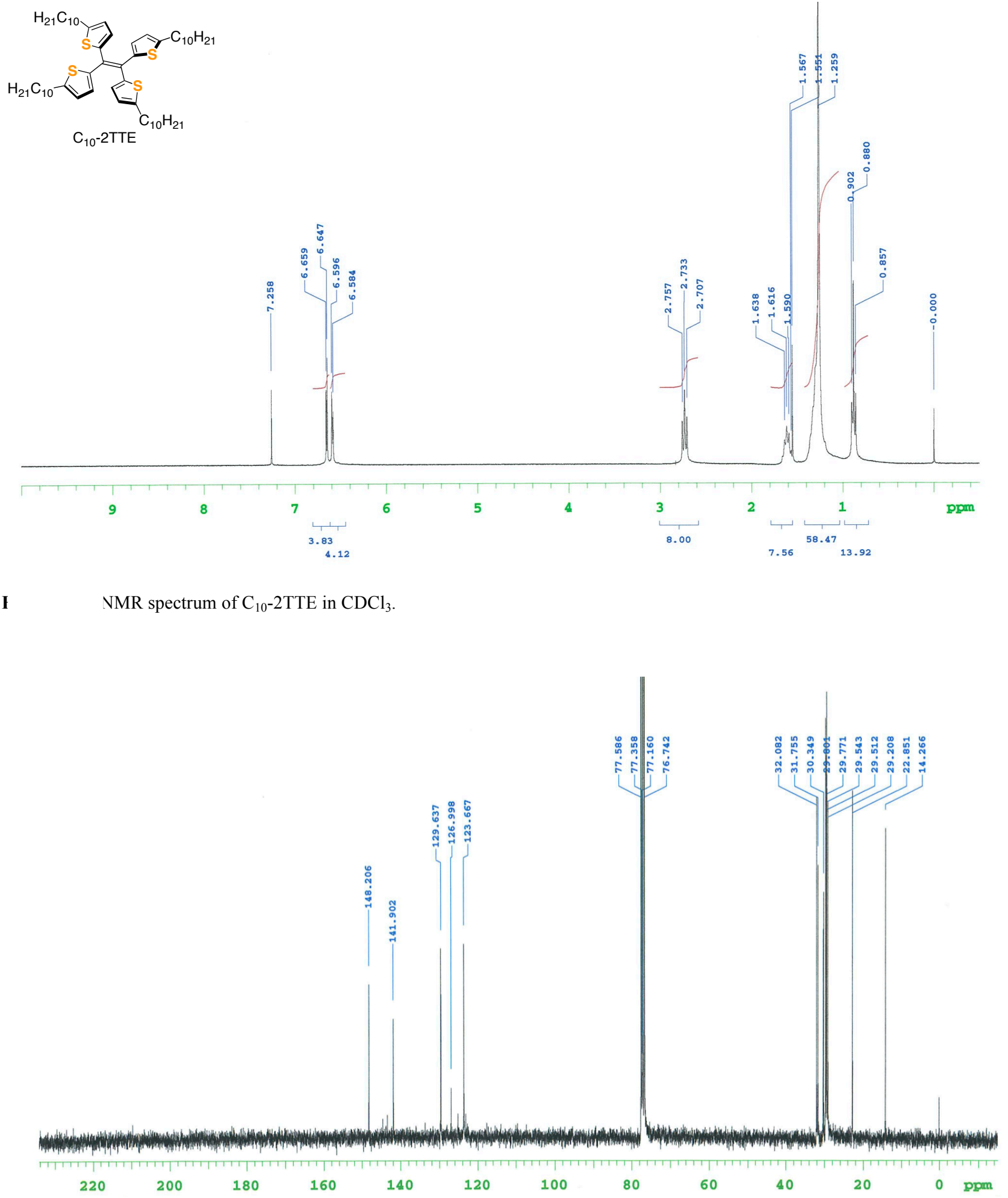

Fig. S26. ${ }^{13} \mathrm{C}$ NMR spectrum of $\mathrm{C}_{10}-2 \mathrm{TTE}$ in $\mathrm{CDCl}_{3}$. 


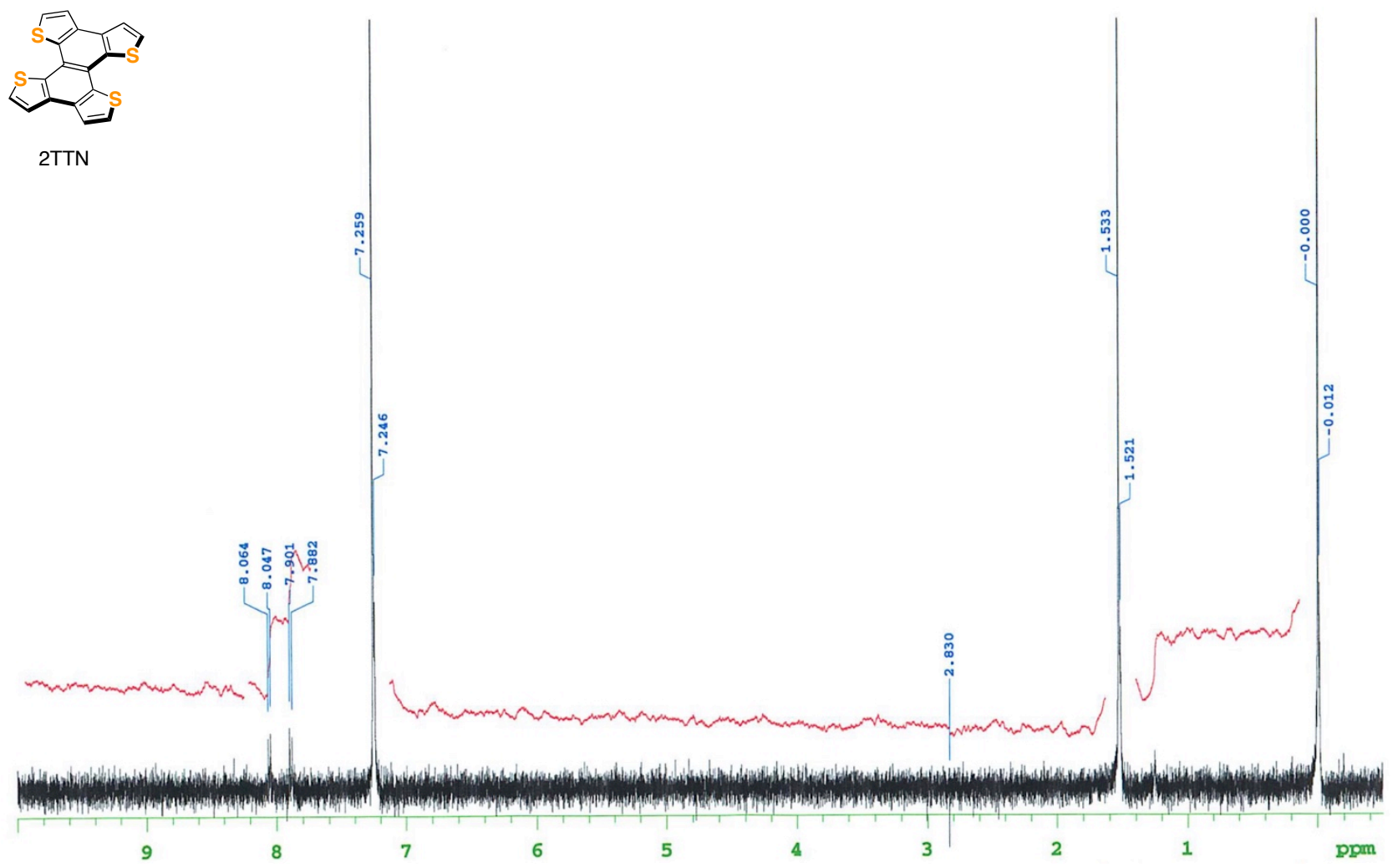

Fig. S27. ${ }^{1} \mathrm{H}$ NMR spectrum of $2 \mathrm{TTN}$ in $\mathrm{CDCl}_{3}$. 

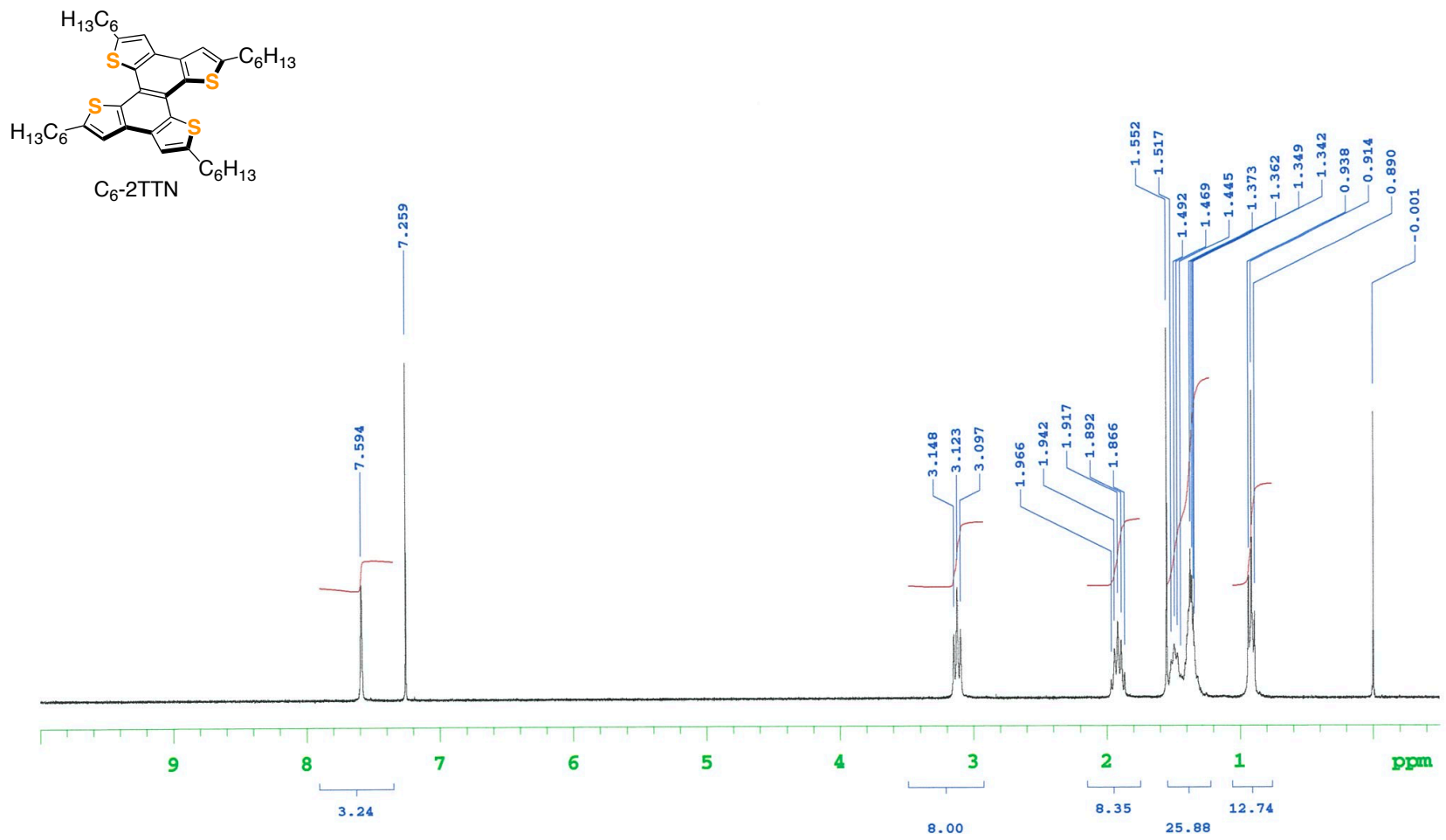

Fig. S28. ${ }^{1} \mathrm{H}$ NMR spectrum of $\mathrm{C}_{6}-2 \mathrm{TTN}$ in $\mathrm{CDCl}_{3}$.

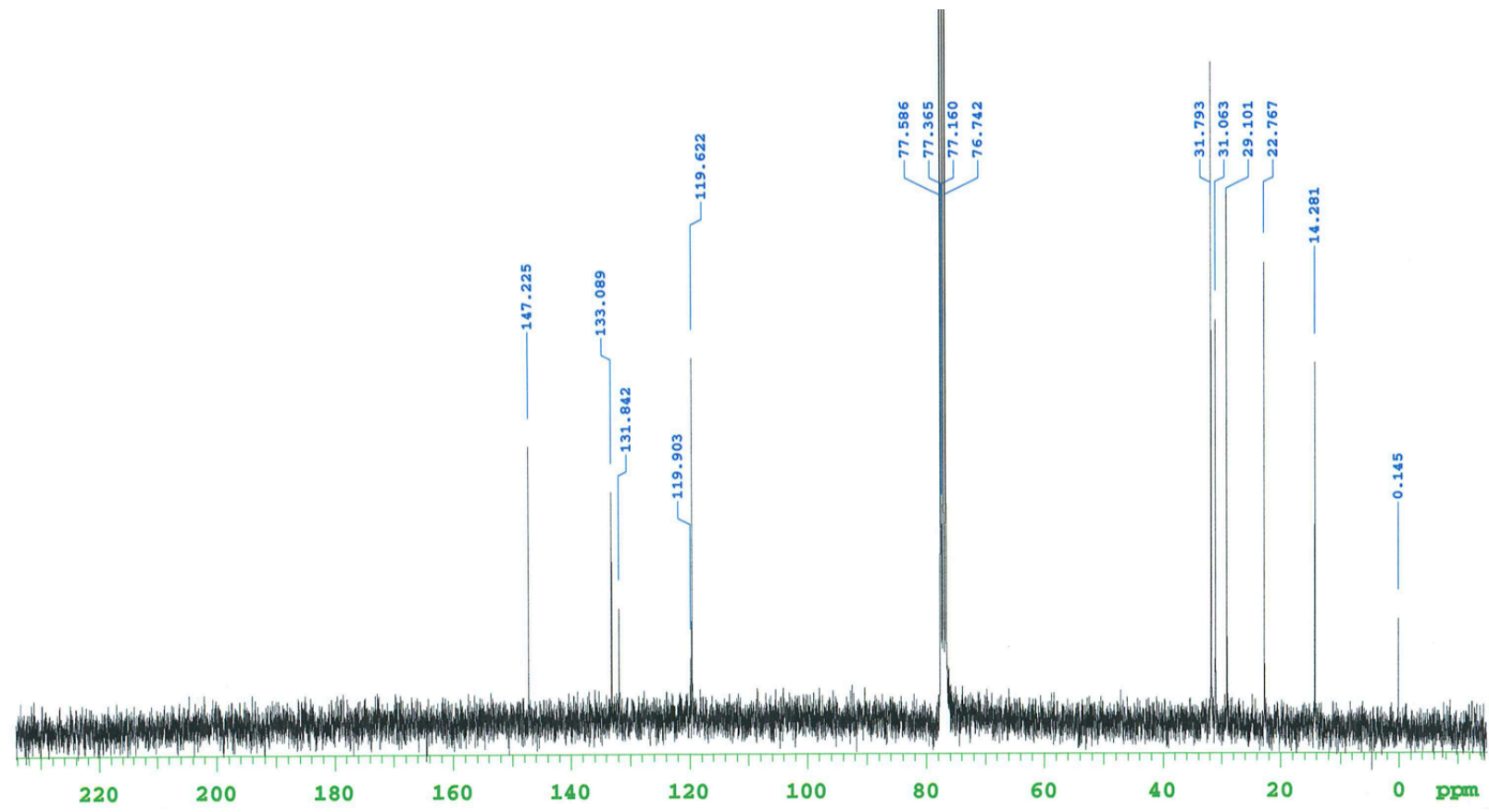

Fig. S29. ${ }^{13} \mathrm{C}$ NMR spectrum of $\mathrm{C}_{6}-2 \mathrm{TTN}$ in $\mathrm{CDCl}_{3}$. 


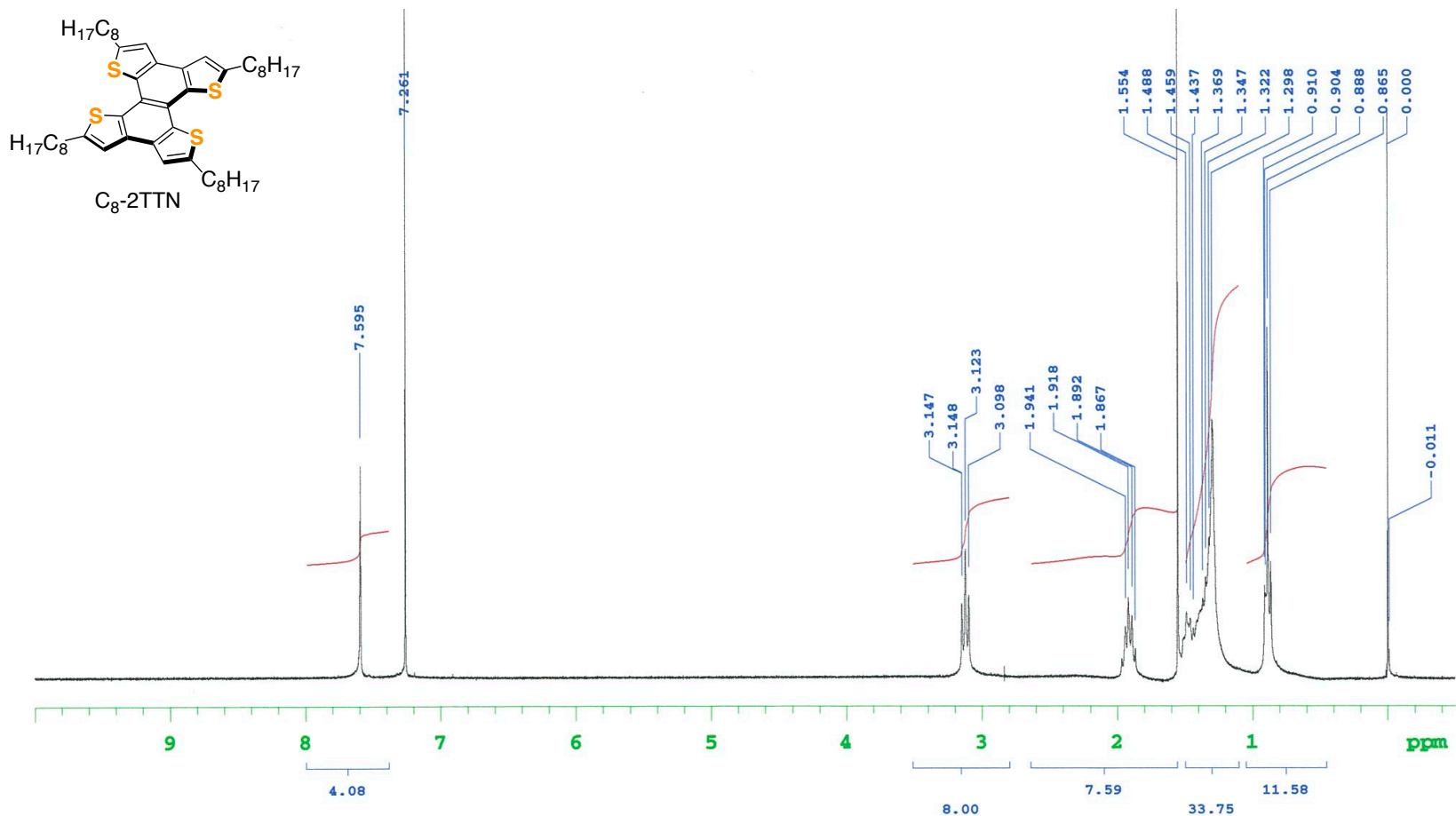

Fig. S30. ${ }^{1} \mathrm{H}$ NMR spectrum of $\mathrm{C}_{8}-2 \mathrm{TTN}$ in $\mathrm{CDCl}_{3}$.

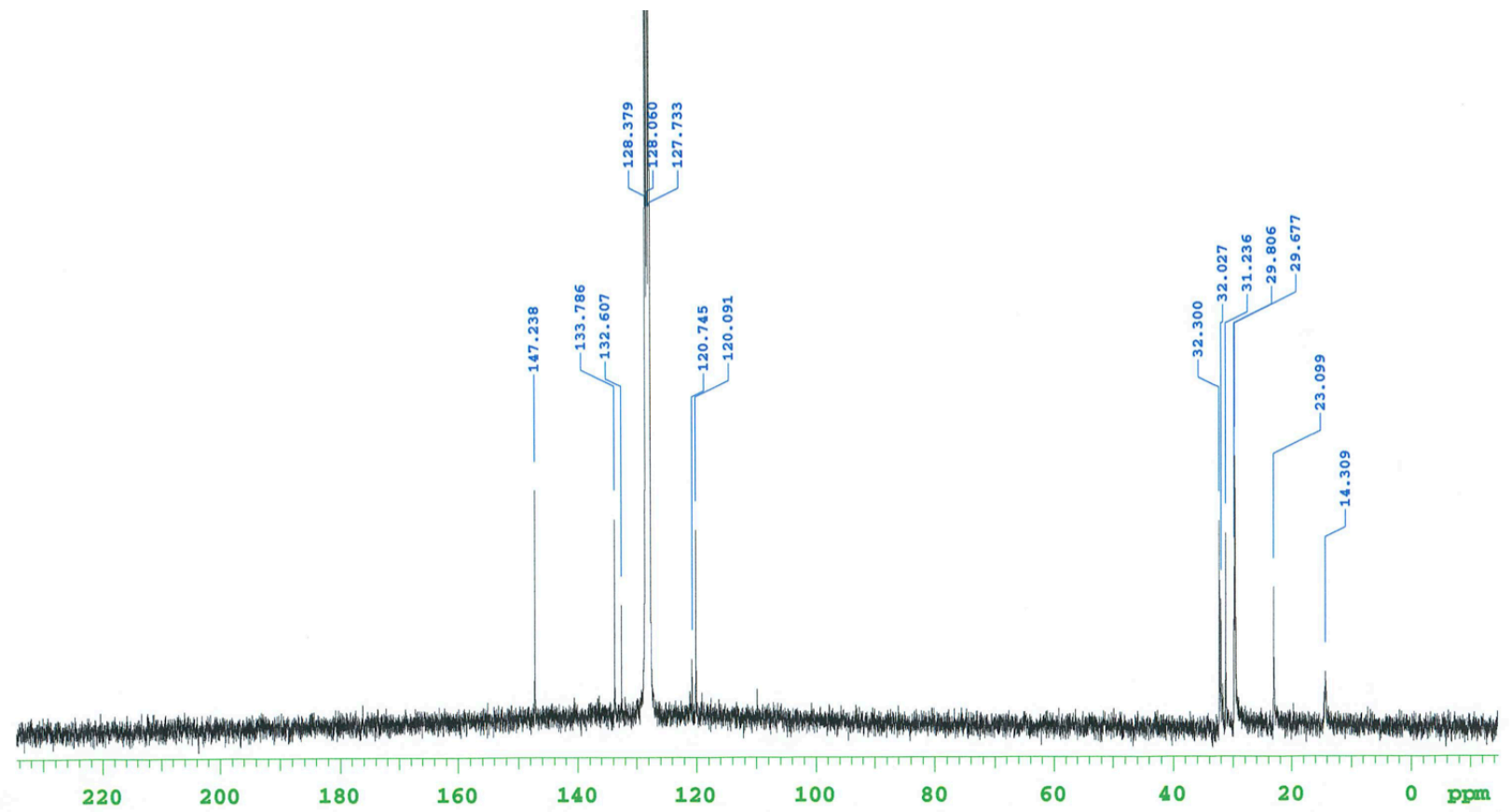

Fig. S31. ${ }^{13} \mathrm{C}$ NMR spectrum of $\mathrm{C}_{8}-2 \mathrm{TTN}$ in $\mathrm{C}_{6} \mathrm{D}_{6}$. 

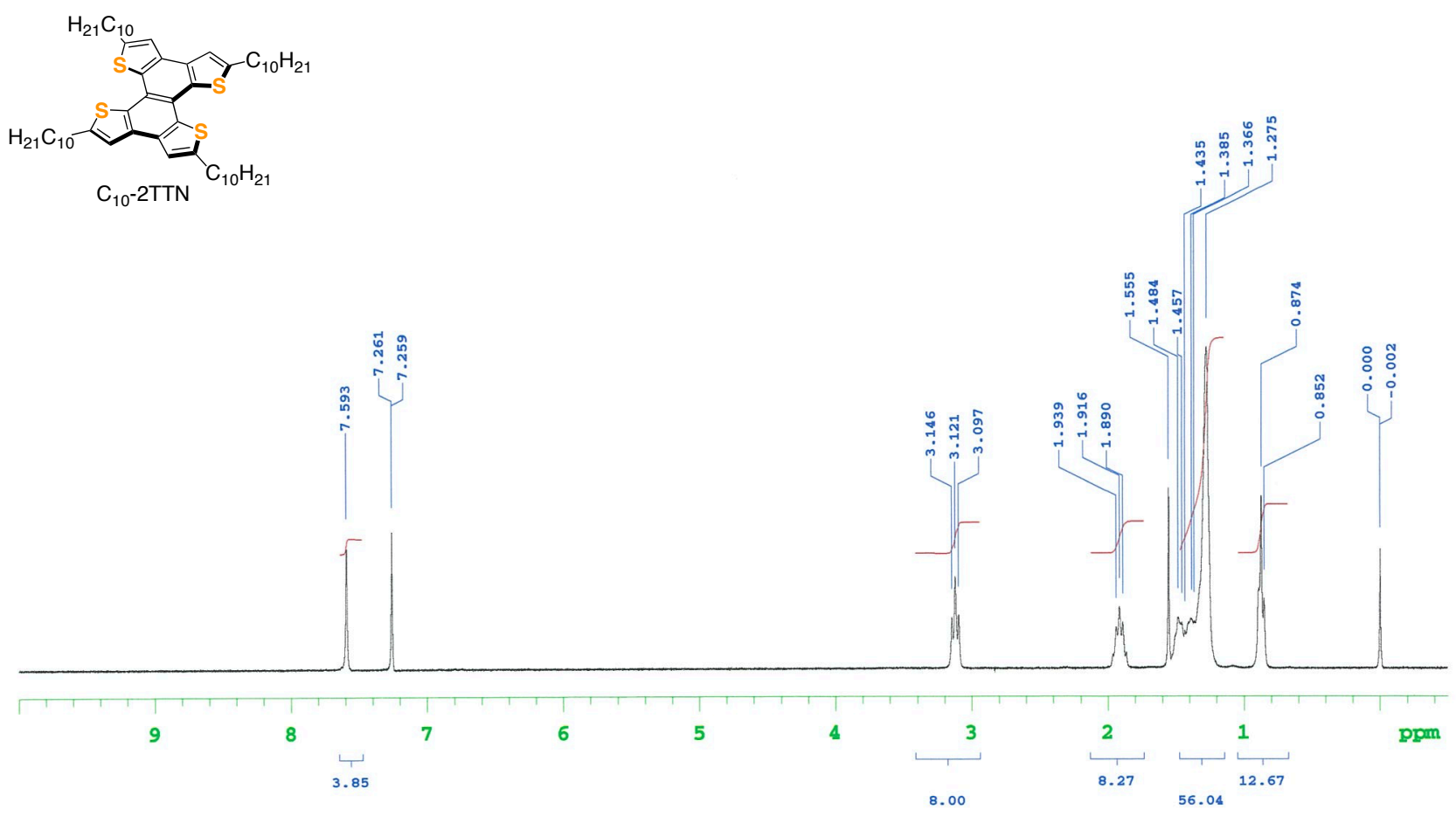

Fig. S32. ${ }^{1} \mathrm{H}$ NMR spectrum of $\mathrm{C}_{10}-2 \mathrm{TTN}$ in $\mathrm{CDCl}_{3}$.

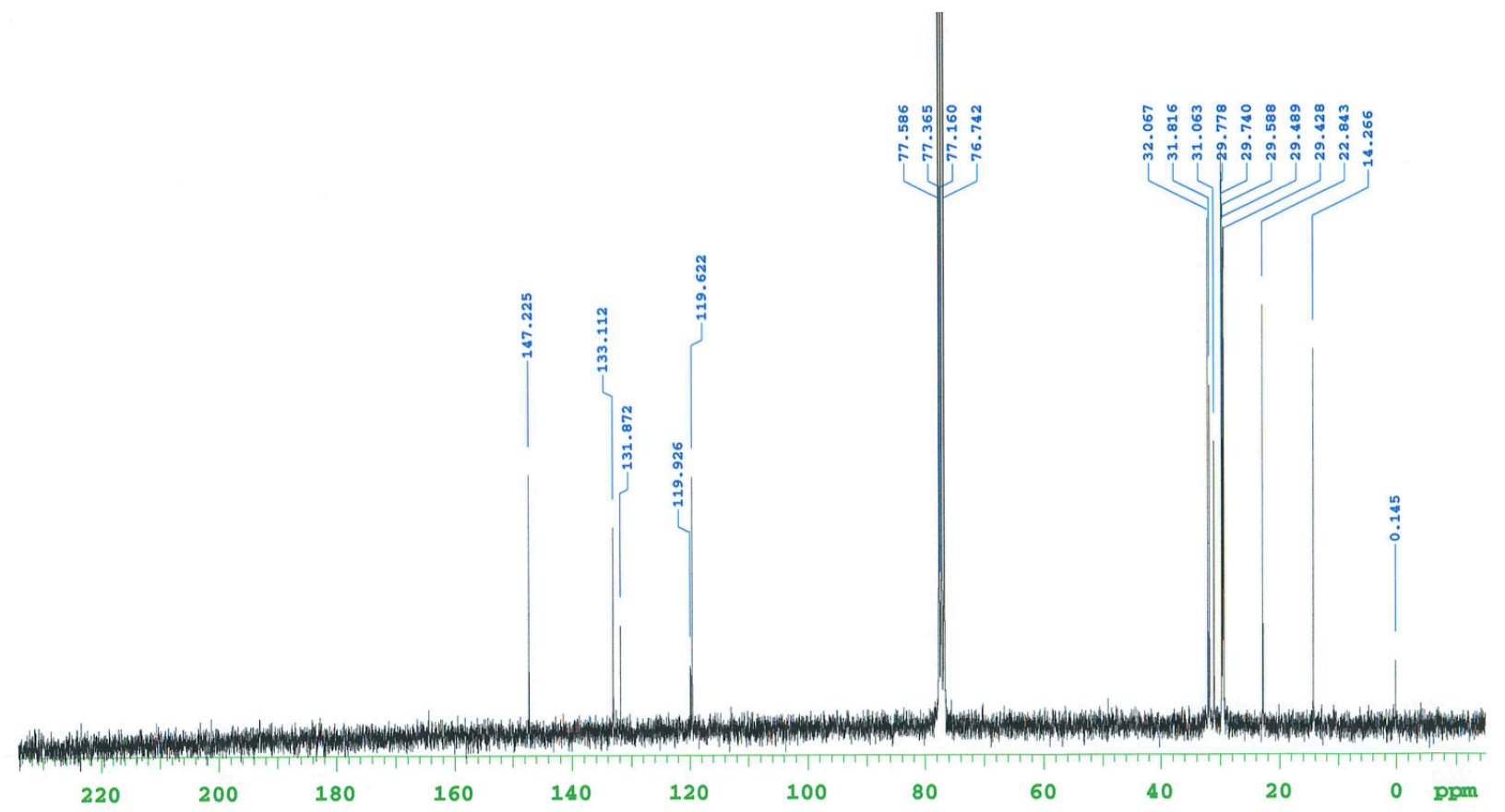

Fig. S33. ${ }^{13} \mathrm{C}$ NMR spectrum of $\mathrm{C}_{10}-2 \mathrm{TTN}$ in $\mathrm{CDCl}_{3}$. 


\section{The Details of DFT Calculations}

Geometry optimizations were performed with the $6-31 \mathrm{G}(\mathrm{d}, \mathrm{p})^{\mathrm{S} 1}$ basis set using B3LYP method. ${ }^{\mathrm{S} 2}$ All the calculations were performed on Gaussian 09 program. No imaginary frequency was found.

Table S1. The Cartesian Coordinates (in $\AA$ ) of 3 TTN Optimized with the B3LYP/6-31G(d,p) Method (The sum of electronic and zero-point energies is -2283.311697 Hartree.)

\begin{tabular}{|c|c|c|c|}
\hline & $\mathrm{X}$ & $\mathrm{Y}$ & Z \\
\hline $\mathrm{C} 1$ & 0.000000 & 0.715911 & 0.000000 \\
\hline $\mathrm{C} 2$ & 0.000000 & -0.715911 & 0.000000 \\
\hline $\mathrm{C} 3$ & -1.256948 & -1.411183 & -0.151483 \\
\hline $\mathrm{C} 4$ & 1.256948 & 1.411183 & -0.151483 \\
\hline $\mathrm{C} 5$ & 1.256947 & -1.411183 & 0.151483 \\
\hline C6 & -1.256948 & 1.411183 & 0.151483 \\
\hline $\mathrm{C} 7$ & -1.509651 & -2.803750 & -0.437412 \\
\hline $\mathrm{C} 8$ & -1.509651 & 2.803751 & 0.437412 \\
\hline C9 & -2.829332 & -3.106589 & -0.556405 \\
\hline $\mathrm{C} 10$ & -2.829332 & 3.106589 & 0.556405 \\
\hline $\mathrm{S} 11$ & -3.861110 & -1.718701 & -0.341727 \\
\hline $\mathrm{S} 12$ & -3.861110 & 1.718700 & 0.341727 \\
\hline $\mathrm{C} 13$ & -2.464418 & 0.694862 & 0.103457 \\
\hline $\mathrm{C} 14$ & -2.464418 & -0.694862 & -0.103457 \\
\hline H15 & -3.268918 & 4.067611 & 0.783891 \\
\hline H16 & -3.268918 & -4.067611 & -0.783891 \\
\hline H17 & -0.742619 & 3.541869 & 0.615674 \\
\hline H18 & -0.742618 & -3.541869 & -0.615673 \\
\hline $\mathrm{C} 19$ & 2.464418 & -0.694862 & 0.103457 \\
\hline $\mathrm{C} 20$ & 1.509651 & -2.803751 & 0.437413 \\
\hline $\mathrm{C} 21$ & 2.829332 & -3.106589 & 0.556406 \\
\hline S22 & 3.861109 & -1.718700 & 0.341727 \\
\hline $\mathrm{H} 23$ & 0.742619 & -3.541869 & 0.615675 \\
\hline $\mathrm{H} 24$ & 3.268918 & -4.067612 & 0.783892 \\
\hline $\mathrm{C} 25$ & 2.464417 & 0.694862 & -0.103458 \\
\hline $\mathrm{C} 26$ & 1.509652 & 2.803751 & -0.437412 \\
\hline $\mathrm{C} 27$ & 2.829332 & 3.106590 & -0.556405 \\
\hline S28 & 3.861110 & 1.718700 & -0.341728 \\
\hline $\mathrm{H} 29$ & 0.742619 & 3.541870 & -0.615673 \\
\hline $\mathrm{H} 30$ & 3.268919 & 4.067612 & -0.783890 \\
\hline
\end{tabular}

Table S2. The Cartesian Coordinates (in $\AA$ ) of $3 \mathrm{TTN}^{\circ+}$ Optimized with the UB3LYP/6-31G(d,p) Method (The sum of electronic and zero-point energies is -2283.065910 Hartree.)

\begin{tabular}{crrr}
\hline & $\mathrm{X}$ & $\mathrm{Y}$ & \multicolumn{1}{c}{$\mathrm{Z}$} \\
\hline $\mathrm{C} 1$ & 0.000000 & 0.000000 & -0.715999 \\
$\mathrm{C} 2$ & 0.000000 & 0.000000 & 0.715999 \\
$\mathrm{C} 3$ & 1.251311 & 0.155851 & 1.411895 \\
$\mathrm{C} 4$ & -1.251311 & 0.155851 & -1.411895 \\
$\mathrm{C} 5$ & -1.251311 & -0.155851 & 1.411895 \\
$\mathrm{C} 6$ & 1.251311 & -0.155851 & -1.411895 \\
$\mathrm{C} 7$ & 1.505319 & 0.443574 & 2.787982
\end{tabular}

\begin{tabular}{rrrr} 
C8 & 1.505319 & -0.443574 & -2.787982 \\
C9 & 2.837349 & 0.555827 & 3.082382 \\
C10 & 2.837349 & -0.555827 & -3.082382 \\
S11 & 3.878358 & 0.329635 & 1.713518 \\
S12 & 3.878358 & -0.329635 & -1.713518 \\
C13 & 2.480395 & -0.101503 & -0.685399 \\
C14 & 2.480395 & 0.101503 & 0.685399 \\
H15 & 3.272804 & -0.783404 & -4.046541 \\
H16 & 3.272804 & 0.783404 & 4.046541 \\
H17 & 0.748840 & -0.628480 & -3.534649 \\
H18 & 0.748840 & 0.628480 & 3.534649 \\
C19 & -2.480395 & -0.101503 & 0.685399 \\
C20 & -1.505319 & -0.443574 & 2.787982 \\
C21 & -2.837349 & -0.555827 & 3.082382 \\
S22 & -3.878358 & -0.329635 & 1.713518 \\
H23 & -0.748840 & -0.628480 & 3.534649 \\
H24 & -3.272804 & -0.783404 & 4.046541 \\
C25 & -2.480395 & 0.101503 & -0.685399 \\
C26 & -1.505319 & 0.443574 & -2.787982 \\
C27 & -2.837349 & 0.555827 & -3.082382 \\
S28 & -3.878358 & 0.329635 & -1.713518 \\
H29 & -0.748840 & 0.628480 & -3.534649 \\
H30 & -3.272804 & 0.783404 & -4.046541 \\
\hline & & &
\end{tabular}

Table S3. The Cartesian Coordinates (in Å) of 2TTN Optimized with the B3LYP/6-31G(d,p) Method (The sum of electronic and zero-point energies is -2283.322039 Hartree.)

\begin{tabular}{rrrr}
\hline & \multicolumn{1}{c}{$\mathrm{X}$} & \multicolumn{1}{c}{$\mathrm{Y}$} & $\mathrm{Z}$ \\
\hline $\mathrm{C} 1$ & 0.000000 & 0.721593 & 0.000000 \\
$\mathrm{C} 2$ & 0.000000 & -0.721587 & 0.000000 \\
$\mathrm{C} 3$ & 1.265818 & -1.391366 & 0.000081 \\
$\mathrm{C} 4$ & -1.265821 & 1.391369 & 0.000081 \\
$\mathrm{C} 5$ & -1.265817 & -1.391366 & -0.000080 \\
$\mathrm{C} 6$ & 1.265821 & 1.391370 & -0.000081 \\
$\mathrm{~S} 7$ & 1.546960 & -3.130567 & 0.000286 \\
$\mathrm{~S} 8$ & 1.546973 & 3.130570 & -0.000288 \\
$\mathrm{C} 9$ & 3.271605 & -2.902795 & 0.000325 \\
$\mathrm{C} 10$ & 3.271617 & 2.902791 & -0.000323 \\
$\mathrm{C} 11$ & 3.625386 & -1.590688 & 0.000202 \\
$\mathrm{C} 12$ & 3.625392 & 1.590683 & -0.000202 \\
$\mathrm{C} 13$ & 2.492994 & 0.711827 & -0.000063 \\
$\mathrm{C} 14$ & 2.492992 & -0.711828 & 0.000063 \\
$\mathrm{H} 15$ & 3.918295 & 3.769426 & -0.000444 \\
$\mathrm{H} 16$ & 3.918279 & -3.769433 & 0.000447 \\
$\mathrm{C} 17$ & -2.492992 & -0.711828 & -0.000063 \\
$\mathrm{~S} 18$ & -1.546960 & -3.130567 & -0.000285 \\
$\mathrm{C} 19$ & -3.271605 & -2.902796 & -0.000327
\end{tabular}




\begin{tabular}{lrrr}
$\mathrm{C} 20$ & -3.625386 & -1.590689 & -0.000202 \\
$\mathrm{H} 21$ & -3.918279 & -3.769433 & -0.000451 \\
$\mathrm{C} 22$ & -2.492994 & 0.711827 & 0.000064 \\
$\mathrm{~S} 23$ & -1.546973 & 3.130569 & 0.000286 \\
$\mathrm{C} 24$ & -3.271618 & 2.902791 & 0.000325 \\
$\mathrm{C} 25$ & -3.625392 & 1.590683 & 0.000202 \\
$\mathrm{H} 26$ & -3.918295 & 3.769426 & 0.000448 \\
$\mathrm{H} 27$ & 4.653807 & -1.250107 & 0.000214 \\
$\mathrm{H} 28$ & 4.653811 & 1.250097 & -0.000214 \\
$\mathrm{H} 29$ & -4.653812 & 1.250097 & 0.000215 \\
$\mathrm{H} 30$ & -4.653807 & -1.250107 & -0.000214 \\
\hline
\end{tabular}

Table S4. The Cartesian Coordinates (in $\AA$ ) of $2 \mathrm{TTN}^{\circ+}$ Optimized with the UB3LYP/6-31G(d,p) Method (The sum of electronic and zero-point energies is -2283.322039 Hartree.)

\begin{tabular}{rrrr}
\hline & $\mathrm{X}$ & $\mathrm{Y}$ & $\mathrm{Z}$ \\
\hline $\mathrm{C} 1$ & -0.000002 & 0.743090 & 0.000001 \\
$\mathrm{C} 2$ & 0.000002 & -0.743101 & 0.000001 \\
$\mathrm{C} 3$ & 1.259818 & -1.402727 & 0.000824 \\
$\mathrm{C} 4$ & -1.259813 & 1.402721 & 0.000826 \\
$\mathrm{C} 5$ & -1.259810 & -1.402734 & -0.000824 \\
$\mathrm{C} 6$ & 1.259805 & 1.402728 & -0.000823 \\
$\mathrm{~S} 7$ & 1.548567 & -3.141845 & 0.002569 \\
$\mathrm{~S} 8$ & 1.548528 & 3.141850 & -0.002574 \\
$\mathrm{C} 9$ & 3.254693 & -2.906099 & 0.002786 \\
$\mathrm{C} 10$ & 3.254657 & 2.906123 & -0.002786 \\
$\mathrm{C} 11$ & 3.613843 & -1.581191 & 0.001684 \\
$\mathrm{C} 12$ & 3.613825 & 1.581219 & -0.001680 \\
$\mathrm{C} 13$ & 2.490437 & 0.717733 & -0.000609 \\
$\mathrm{C} 14$ & 2.490444 & -0.717719 & 0.000613 \\
$\mathrm{H} 15$ & 3.909695 & 3.767481 & -0.003812 \\
$\mathrm{H} 16$ & 3.909742 & -3.767448 & 0.003809 \\
$\mathrm{C} 17$ & -2.490440 & -0.717732 & -0.000610 \\
$\mathrm{~S} 18$ & -1.548550 & -3.141854 & -0.002574 \\
$\mathrm{C} 19$ & -3.254678 & -2.906116 & -0.002783 \\
$\mathrm{C} 20$ & -3.613835 & -1.581210 & -0.001679 \\
$\mathrm{H} 21$ & -3.909722 & -3.767469 & -0.003808 \\
$\mathrm{C} 22$ & -2.490440 & 0.717720 & 0.000611 \\
$\mathrm{~S} 23$ & -1.548546 & 3.141842 & 0.002571 \\
$\mathrm{C} 24$ & -3.254673 & 2.906105 & 0.002784 \\
$\mathrm{C} 25$ & -3.613833 & 1.581199 & 0.001681 \\
$\mathrm{H} 26$ & -3.909715 & 3.767460 & 0.003809 \\
$\mathrm{H} 27$ & 4.644123 & -1.248535 & 0.001729 \\
$\mathrm{H} 28$ & 4.644108 & 1.248576 & -0.001721 \\
\hline & -4.644115 & 1.248551 & 0.001724 \\
$\mathrm{H} 30$ & -1.248559 & -0.001720 \\
\hline
\end{tabular}

Table S5. The Cartesian Coordinates (in $\AA$ ) of $\mathrm{C}_{6}-2 \mathrm{TTN}$ Optimized with the B3LYP/6-31G(d,p) Method (The sum of electronic and zero-point energies is -3227.13718689 Hartree.)

\begin{tabular}{|c|c|c|c|}
\hline & $X$ & $\mathrm{Y}$ & Z \\
\hline $\mathrm{C} 1$ & 0.000000 & 0.000000 & 0.000000 \\
\hline $\mathrm{C} 2$ & 0.000000 & 0.000000 & 1.361900 \\
\hline $\mathrm{C} 3$ & 5.462484 & 0.000000 & -1.717075 \\
\hline $\mathrm{C} 4$ & 0.765990 & 0.009359 & -3.088516 \\
\hline $\mathrm{C} 5$ & 1.402653 & 0.014789 & -4.292541 \\
\hline C6 & 7.802024 & 0.006835 & -2.704268 \\
\hline C7 & 6.397184 & -0.033027 & 2.947993 \\
\hline $\mathrm{C} 8$ & 4.793261 & -0.011188 & 0.981427 \\
\hline $\mathrm{C} 9$ & 6.488866 & -0.005099 & -0.763643 \\
\hline $\mathrm{C} 10$ & 6.146975 & -0.013257 & 0.617424 \\
\hline $\mathrm{C} 11$ & 1.652126 & 0.003602 & -1.962491 \\
\hline $\mathrm{C} 12$ & 1.309561 & 0.000200 & -0.581241 \\
\hline $\mathrm{C} 13$ & 3.005866 & 0.002835 & -2.326016 \\
\hline $\mathrm{C} 14$ & 2.336239 & -0.002174 & 0.372766 \\
\hline $\mathrm{C} 15$ & 4.073991 & -0.002133 & -1.373645 \\
\hline $\mathrm{C} 16$ & 3.725551 & -0.004115 & 0.029742 \\
\hline S17 & 3.147585 & 0.011891 & -4.083594 \\
\hline S18 & 1.640624 & -0.000859 & 1.992662 \\
\hline S19 & 6.161190 & 0.008595 & -3.336154 \\
\hline S20 & 4.651811 & -0.024772 & 2.738669 \\
\hline $\mathrm{C} 21$ & 7.801032 & -0.000124 & -1.342486 \\
\hline $\mathrm{C} 22$ & 7.033719 & -0.025889 & 1.743796 \\
\hline $\mathrm{H} 23$ & -0.914667 & 0.003831 & -0.582488 \\
\hline $\mathrm{H} 24$ & -0.315022 & 0.013382 & -3.001694 \\
\hline $\mathrm{H} 25$ & 8.708775 & -0.000387 & -0.751816 \\
\hline $\mathrm{H} 26$ & 8.114633 & -0.034360 & 1.657329 \\
\hline $\mathrm{C} 27$ & -1.178255 & -0.013367 & 2.293157 \\
\hline $\mathrm{C} 28$ & -1.335257 & -1.316140 & 3.103223 \\
\hline $\mathrm{H} 29$ & -2.079842 & 0.150582 & 1.690289 \\
\hline $\mathrm{H} 30$ & -1.115787 & 0.834551 & 2.988897 \\
\hline $\mathrm{C} 31$ & -2.569496 & -1.304446 & 4.012233 \\
\hline H32 & -1.391194 & -2.163223 & 2.407804 \\
\hline H33 & -0.434428 & -1.477551 & 3.709176 \\
\hline C34 & -2.734208 & -2.589649 & 4.832630 \\
\hline H35 & -2.510628 & -0.443775 & 4.693908 \\
\hline H36 & -3.469943 & -1.145571 & 3.401616 \\
\hline H37 & -2.792869 & -3.450733 & 4.151782 \\
\hline H38 & -1.833996 & -2.748343 & 5.443282 \\
\hline C39 & 0.794965 & 0.001190 & -5.666052 \\
\hline $\mathrm{C} 40$ & 0.988691 & -1.323038 & -6.432389 \\
\hline H41 & 1.203798 & 0.825368 & -6.266109 \\
\hline $\mathrm{H} 42$ & -0.277485 & 0.203751 & -5.556466 \\
\hline $\mathrm{C} 43$ & 0.311937 & -1.318669 & -7.808250 \\
\hline $\mathrm{H} 44$ & 2.061927 & -1.520689 & -6.549960 \\
\hline $\mathrm{H} 45$ & 0.591558 & -2.146171 & -5.824734 \\
\hline $\mathrm{C} 46$ & 0.510404 & -2.631107 & -8.578587 \\
\hline $\mathrm{H} 47$ & -0.759829 & -1.118248 & -7.678817 \\
\hline $\mathrm{H} 48$ & 0.707650 & -0.485142 & -8.406752 \\
\hline H49 & 1.587406 & -2.826938 & -8.667915 \\
\hline $\mathrm{H} 50$ & 0.099676 & -3.462888 & -7.988380 \\
\hline
\end{tabular}




\begin{tabular}{|c|c|c|c|c|c|c|c|}
\hline C51 & 8.963244 & 0.008253 & -3.662442 & $\mathrm{H} 102$ & 6.637779 & 6.454789 & 5.661311 \\
\hline C52 & 10.336563 & 0.114201 & -2.986539 & \multirow{5}{*}{\multicolumn{4}{|c|}{$\begin{array}{l}\text { Table S6. The Cartesian Coordinates (in } \AA \text { ) of } \mathrm{C}_{6}-2 \mathrm{TTN}^{*+} \\
\text { Optimized with the UB3LYP/6-31G(d,p) Method (The sum } \\
\text { of electronic and zero-point energies is }-3226.91055070 \\
\text { Hartree.) }\end{array}$}} \\
\hline $\mathrm{H} 53$ & 8.845574 & 0.839380 & -4.371642 & & & & \\
\hline H54 & 8.930531 & -0.905577 & -4.272610 & & & & \\
\hline C55 & 11.494067 & 0.124787 & -3.992980 & & & & \\
\hline H56 & 10.371956 & 1.027461 & -2.378371 & & & & \\
\hline H57 & 10.467006 & -0.726302 & -2.292489 & & $X$ & $\mathrm{Y}$ & $\mathrm{Z}$ \\
\hline C58 & 12.869119 & 0.244185 & -3.321951 & $\mathrm{C} 1$ & 0.000000 & 0.000000 & 0.000000 \\
\hline H59 & 11.449877 & -0.789174 & -4.599517 & $\mathrm{C} 2$ & 0.000000 & 0.000000 & 1.378300 \\
\hline H60 & 11.360136 & 0.962332 & -4.692609 & $\mathrm{C} 3$ & 5.441605 & 0.000000 & -1.724088 \\
\hline H61 & 12.874409 & 1.142336 & -2.689670 & $\mathrm{C} 4$ & 0.758081 & 0.009924 & -3.068117 \\
\hline $\mathrm{H} 62$ & 13.015323 & -0.606734 & -2.641009 & $\mathrm{C} 5$ & 1.399562 & 0.017284 & -4.287454 \\
\hline C63 & 6.996958 & -0.039473 & 4.326121 & C6 & 7.768164 & -0.003321 & -2.710671 \\
\hline C64 & 6.814710 & 1.273654 & 5.119733 & $\mathrm{C} 7$ & 6.368057 & -0.028957 & 2.954885 \\
\hline H65 & 8.067536 & -0.254918 & 4.221938 & $\mathrm{C} 8$ & 4.769685 & -0.012531 & 0.996514 \\
\hline H66 & 6.570380 & -0.866736 & 4.907647 & C9 & 6.471947 & -0.002781 & -0.764625 \\
\hline C67 & 7.549417 & 2.471372 & 4.504987 & $\mathrm{C} 10$ & 6.127081 & -0.010185 & 0.628502 \\
\hline H68 & 5.743488 & 1.489973 & 5.207948 & $\mathrm{C} 11$ & 1.640620 & 0.002477 & -1.961451 \\
\hline H69 & 7.181594 & 1.108049 & 6.141610 & $\mathrm{C} 12$ & 1.295768 & -0.002035 & -0.568264 \\
\hline $\mathrm{C} 70$ & 7.438532 & 3.771764 & 5.317377 & $\mathrm{C} 13$ & 2.998391 & 0.002810 & -2.329614 \\
\hline $\mathrm{H} 71$ & 8.611950 & 2.211232 & 4.399388 & C14 & 2.326441 & -0.005684 & 0.390842 \\
\hline $\mathrm{H} 72$ & 7.177219 & 2.643766 & 3.486913 & C15 & 4.062357 & -0.002127 & -1.387661 \\
\hline $\mathrm{H} 73$ & 7.800087 & 3.594622 & 6.340939 & $\mathrm{C} 16$ & 3.705573 & -0.006373 & 0.053884 \\
\hline $\mathrm{H} 74$ & 8.120898 & 4.514733 & 4.882549 & S17 & 3.130520 & 0.014373 & -4.087251 \\
\hline $\mathrm{C} 75$ & -3.967800 & -2.580844 & 5.743730 & $\mathrm{~S} 18$ & 1.624200 & -0.001984 & 2.008286 \\
\hline $\mathrm{C} 76$ & -4.123844 & -3.865757 & 6.562539 & S19 & 6.143986 & -0.002028 & -3.340992 \\
\hline $\mathrm{H} 77$ & -4.867320 & -2.424085 & 5.132999 & $\mathrm{~S} 20$ & 4.635984 & -0.028067 & 2.753856 \\
\hline $\mathrm{H} 78$ & -3.909196 & -1.719162 & 6.422531 & $\mathrm{C} 21$ & 7.767911 & -0.003356 & -1.332471 \\
\hline H79 & -5.009655 & -3.826802 & 7.204730 & $\mathrm{C} 22$ & 7.009349 & -0.018358 & 1.736039 \\
\hline $\mathrm{H} 80$ & -4.223917 & -4.741557 & 5.911420 & $\mathrm{H} 23$ & -0.917542 & 0.004964 & -0.576251 \\
\hline H81 & -3.253214 & -4.032905 & 7.206792 & $\mathrm{H} 24$ & -0.322201 & 0.013918 & -2.984765 \\
\hline $\mathrm{C} 82$ & -0.114578 & -2.651879 & -9.983287 & $\mathrm{H} 25$ & 8.685348 & -0.008054 & -0.756051 \\
\hline $\mathrm{C} 83$ & -1.646148 & -2.585236 & -9.999026 & $\mathrm{H} 26$ & 8.089469 & -0.019971 & 1.653100 \\
\hline H84 & 0.205946 & -3.568627 & -10.494289 & $\mathrm{C} 27$ & -1.181730 & -0.038789 & 2.298416 \\
\hline H85 & 0.293818 & -1.819246 & -10.572419 & $\mathrm{C} 28$ & -1.438098 & -1.433771 & 2.917581 \\
\hline H86 & -2.019979 & -1.649099 & -9.571843 & $\mathrm{H} 29$ & -2.062830 & 0.275238 & 1.727795 \\
\hline H87 & -2.032526 & -2.655123 & -11.021099 & $\mathrm{H} 30$ & -1.051516 & 0.694129 & 3.103789 \\
\hline H88 & -2.082105 & -3.409522 & -9.422513 & $\mathrm{C} 31$ & -2.671472 & -1.449483 & 3.828041 \\
\hline C89 & 14.055263 & 0.319135 & -4.297165 & H32 & -1.559108 & -2.166225 & 2.109945 \\
\hline C90 & 14.287100 & -0.956094 & -5.115780 & H33 & -0.552693 & -1.743981 & 3.486892 \\
\hline H91 & 14.964042 & 0.543488 & -3.724387 & C34 & -2.938590 & -2.824819 & 4.452497 \\
\hline H92 & 13.909321 & 1.169407 & -4.977518 & H35 & -2.543702 & -0.704685 & 4.625872 \\
\hline H93 & 13.440571 & -1.181573 & -5.772223 & H36 & -3.552535 & -1.132571 & 3.253014 \\
\hline H94 & 15.174918 & -0.860961 & -5.749275 & H37 & -3.066899 & -3.568471 & 3.653410 \\
\hline H95 & 14.438079 & -1.822330 & -4.461017 & H38 & -2.055063 & -3.142285 & 5.024002 \\
\hline C96 & 6.029669 & 4.378514 & 5.376459 & C39 & 0.780536 & -0.010831 & -5.651719 \\
\hline C97 & 5.986630 & 5.709865 & 6.132808 & $\mathrm{C} 40$ & 0.885983 & -1.385473 & -6.353687 \\
\hline H98 & 5.336378 & 3.672837 & 5.851029 & H41 & 1.242493 & 0.754823 & -6.286981 \\
\hline H99 & 5.658890 & 4.525194 & 4.352989 & $\mathrm{H} 42$ & -0.274599 & 0.265214 & -5.545564 \\
\hline H100 & 4.973261 & 6.123356 & 6.159207 & $\mathrm{C} 43$ & 0.181239 & -1.400104 & -7.715465 \\
\hline H101 & 6.322821 & 5.588341 & 7.168975 & H44 & 1.944401 & -1.647091 & -6.479443 \\
\hline
\end{tabular}




\begin{tabular}{|c|c|c|c|c|c|c|c|}
\hline $\mathrm{H} 45$ & 0.452620 & -2.153678 & -5.701243 & C96 & 5.644443 & 4.210251 & 5.634251 \\
\hline $\mathrm{C} 46$ & 0.290642 & -2.756772 & -8.425405 & C97 & 5.513270 & 5.492965 & 6.461203 \\
\hline $\mathrm{H} 47$ & -0.874050 & -1.134279 & -7.574087 & H98 & 5.040601 & 3.420360 & 6.099431 \\
\hline $\mathrm{H} 48$ & 0.611503 & -0.618464 & -8.357455 & H99 & 5.217334 & 4.372204 & 4.634566 \\
\hline H49 & 1.352997 & -3.013804 & -8.533101 & H100 & 4.468453 & 5.806428 & 6.549587 \\
\hline $\mathrm{H} 50$ & -0.148319 & -3.535963 & -7.786158 & H101 & 5.904578 & 5.352442 & 7.474737 \\
\hline $\mathrm{C} 51$ & 8.950446 & 0.035120 & -3.630093 & H102 & 6.071045 & 6.317592 & 6.003802 \\
\hline C52 & 9.207937 & 1.429434 & -4.250297 & \multirow{5}{*}{\multicolumn{4}{|c|}{$\begin{array}{l}\text { Table S7. The Cartesian Coordinates (in } \AA \text { ) of } \mathrm{C}_{8}-2 \mathrm{TTN} \\
\text { Optimized with the B } 3 \mathrm{LYP} / 6-31 \mathrm{G}(\mathrm{d}, \mathrm{p}) \text { Method (The sum of } \\
\text { electronic and zero-point energies is }-3541.66362028 \\
\text { Hartree.) }\end{array}$}} \\
\hline H53 & 8.821017 & -0.698562 & -4.434897 & & & & \\
\hline $\mathrm{H} 54$ & 9.830929 & -0.278468 & -3.058279 & & & & \\
\hline C55 & 10.445938 & 1.444811 & -5.154800 & & & & \\
\hline $\mathrm{H} 56$ & 8.324486 & 1.738175 & -4.823619 & & & & \\
\hline $\mathrm{H} 57$ & 9.325117 & 2.163249 & -3.443195 & & $X$ & $\mathrm{Y}$ & Z \\
\hline $\mathrm{C} 58$ & 10.708218 & 2.823504 & -5.777097 & $\mathrm{C} 1$ & 0.000000 & 0.000000 & 0.000000 \\
\hline H59 & 11.319198 & 1.123866 & -4.572820 & $\mathrm{C} 2$ & 0.000000 & 0.000000 & 1.363300 \\
\hline H60 & 10.320630 & 0.702142 & -5.955413 & $\mathrm{C} 3$ & 5.463980 & 0.000000 & -1.712308 \\
\hline H61 & 9.809259 & 3.141417 & -6.322136 & $\mathrm{C} 4$ & 0.767928 & -0.003753 & -3.086913 \\
\hline H62 & 10.854828 & 3.560460 & -4.974825 & C5 & 1.404572 & -0.010576 & -4.290941 \\
\hline C63 & 6.982187 & -0.041937 & 4.323342 & C6 & 7.800960 & -0.001406 & -2.700444 \\
\hline C64 & 6.695739 & 1.217474 & 5.175198 & $\mathrm{C} 7$ & 6.393744 & -0.045410 & 2.953540 \\
\hline H65 & 8.064038 & -0.164731 & 4.199656 & $\mathrm{C} 8$ & 4.792971 & -0.016785 & 0.985270 \\
\hline H66 & 6.626349 & -0.927142 & 4.865430 & $\mathrm{C} 9$ & 6.490833 & -0.006590 & -0.757775 \\
\hline C67 & 7.298080 & 2.505442 & 4.599988 & $\mathrm{C} 10$ & 6.147200 & -0.016622 & 0.623181 \\
\hline H68 & 5.611960 & 1.324143 & 5.303085 & C11 & 1.653654 & -0.000380 & -1.960556 \\
\hline H69 & 7.105739 & 1.036782 & 6.176199 & $\mathrm{C} 12$ & 1.309764 & 0.001888 & -0.579221 \\
\hline $\mathrm{C} 70$ & 7.100053 & 3.744265 & 5.488640 & $\mathrm{C} 13$ & 3.007238 & -0.002248 & -2.323499 \\
\hline H71 & 8.374286 & 2.348731 & 4.444601 & C14 & 2.337130 & -0.000676 & 0.375807 \\
\hline H72 & 6.871636 & 2.700691 & 3.606316 & $\mathrm{C} 15$ & 4.075090 & -0.002134 & -1.369889 \\
\hline $\mathrm{H} 73$ & 7.520890 & 3.547195 & 6.484672 & C16 & 3.726026 & -0.006181 & 0.032610 \\
\hline $\mathrm{H} 74$ & 7.692397 & 4.568337 & 5.070334 & S17 & 3.149921 & -0.008818 & -4.080423 \\
\hline $\mathrm{C} 75$ & -4.168816 & -2.847774 & 5.368561 & $\mathrm{~S} 18$ & 1.637297 & -0.001143 & 1.992127 \\
\hline C76 & -4.428728 & -4.222645 & 5.990699 & S19 & 6.160552 & 0.006142 & -3.331443 \\
\hline H77 & -5.051332 & -2.530768 & 4.796778 & $\mathrm{~S} 20$ & 4.648757 & -0.039335 & 2.741778 \\
\hline $\mathrm{H} 78$ & -4.039618 & -2.102907 & 6.165270 & $\mathrm{C} 21$ & 7.800949 & -0.007275 & -1.338556 \\
\hline H79 & -5.309270 & -4.204861 & 6.640009 & $\mathrm{C} 22$ & 7.032129 & -0.031412 & 1.750497 \\
\hline H80 & -4.600254 & -4.982018 & 5.219668 & $\mathrm{H} 23$ & -0.912778 & -0.007010 & -0.585785 \\
\hline H81 & -3.576631 & -4.552100 & 6.595636 & $\mathrm{H} 24$ & -0.312937 & -0.008516 & -2.999556 \\
\hline $\mathrm{C} 82$ & -0.375726 & -2.804221 & -9.810626 & $\mathrm{H} 25$ & 8.715182 & -0.017270 & -0.755460 \\
\hline $\mathrm{C} 83$ & -1.900672 & -2.651769 & -9.789001 & $\mathrm{H} 26$ & 8.113073 & -0.037446 & 1.664208 \\
\hline H84 & -0.120438 & -3.760593 & -10.282783 & $\mathrm{C} 27$ & -1.184851 & 0.015097 & 2.293754 \\
\hline H85 & 0.063063 & -2.025666 & -10.449217 & $\mathrm{C} 28$ & -1.900686 & 1.380474 & 2.395490 \\
\hline H86 & -2.212673 & -1.674532 & -9.406003 & $\mathrm{H} 29$ & -0.876864 & -0.308162 & 3.295233 \\
\hline H87 & -2.317409 & -2.753144 & -10.795643 & $\mathrm{H} 30$ & -1.904501 & -0.733385 & 1.940104 \\
\hline H88 & -2.365126 & -3.419759 & -9.159788 & $\mathrm{C} 31$ & -1.072906 & 2.486024 & 3.059639 \\
\hline $\mathrm{C} 89$ & 11.911192 & 2.870745 & -6.733729 & H32 & -2.199070 & 1.699610 & 1.388373 \\
\hline $\mathrm{C} 90$ & 13.267995 & 2.622956 & -6.065029 & H33 & -2.830752 & 1.234977 & 2.961431 \\
\hline H91 & 11.927205 & 3.854927 & -7.217341 & $\mathrm{C} 34$ & -1.818022 & 3.823980 & 3.144599 \\
\hline H92 & 11.759669 & 2.140480 & -7.540101 & H35 & -0.785771 & 2.164169 & 4.071115 \\
\hline H93 & 13.344380 & 1.614064 & -5.646307 & H36 & -0.135219 & 2.626054 & 2.507189 \\
\hline H94 & 14.084159 & 2.736999 & -6.784851 & H37 & -2.091431 & 4.150308 & 2.131081 \\
\hline H95 & 13.443193 & 3.335490 & -5.250759 & H38 & -2.766590 & 3.680305 & 3.681904 \\
\hline
\end{tabular}




\begin{tabular}{|c|c|c|c|}
\hline C39 & 0.795788 & -0.027413 & -5.663931 \\
\hline $\mathrm{C} 40$ & 1.119689 & 1.212984 & -6.522083 \\
\hline H41 & -0.288105 & -0.124081 & -5.536766 \\
\hline $\mathrm{H} 42$ & 1.123125 & -0.926362 & -6.205412 \\
\hline $\mathrm{C} 43$ & 0.514094 & 1.163461 & -7.934751 \\
\hline $\mathrm{H} 44$ & 0.785913 & 2.112067 & -5.990316 \\
\hline H45 & 2.208875 & 1.305548 & -6.611952 \\
\hline $\mathrm{C} 46$ & -1.024598 & 1.176689 & -8.010246 \\
\hline $\mathrm{H} 47$ & 0.912400 & 2.006702 & -8.511665 \\
\hline $\mathrm{H} 48$ & 0.877694 & 0.259327 & -8.441957 \\
\hline H49 & -1.315044 & 1.076939 & -9.064946 \\
\hline $\mathrm{H} 50$ & -1.423159 & 0.284792 & -7.509632 \\
\hline $\mathrm{C} 51$ & 8.977564 & 0.014633 & -3.633906 \\
\hline C52 & 9.178246 & 1.348621 & -4.381703 \\
\hline H53 & 8.882168 & -0.796379 & -4.368509 \\
\hline H54 & 9.874927 & -0.209215 & -3.044143 \\
\hline C55 & 10.387509 & 1.324054 & -5.323925 \\
\hline H56 & 8.271431 & 1.583634 & -4.953700 \\
\hline H57 & 9.292419 & 2.155059 & -3.646090 \\
\hline $\mathrm{C} 58$ & 10.599148 & 2.650808 & -6.066531 \\
\hline H59 & 11.293940 & 1.083608 & -4.749356 \\
\hline H60 & 10.260089 & 0.506908 & -6.046019 \\
\hline H61 & 9.701117 & 2.881259 & -6.657389 \\
\hline H62 & 10.688596 & 3.457234 & -5.326301 \\
\hline C63 & 6.996234 & -0.055527 & 4.329370 \\
\hline C64 & 6.764127 & 1.244926 & 5.127513 \\
\hline H65 & 8.074739 & -0.224344 & 4.219021 \\
\hline H66 & 6.606159 & -0.910665 & 4.895046 \\
\hline C67 & 7.444907 & 1.263606 & 6.505283 \\
\hline H68 & 7.132262 & 2.086862 & 4.529238 \\
\hline H69 & 5.685292 & 1.406688 & 5.250447 \\
\hline $\mathrm{C} 70$ & 6.884972 & 0.253198 & 7.516414 \\
\hline H71 & 8.524157 & 1.094124 & 6.380320 \\
\hline H72 & 7.345884 & 2.273427 & 6.925139 \\
\hline H73 & 5.798739 & 0.397863 & 7.606249 \\
\hline H74 & 7.024461 & -0.770400 & 7.143810 \\
\hline $\mathrm{C} 75$ & -1.011273 & 4.932365 & 3.832258 \\
\hline $\mathrm{C} 76$ & -1.752170 & 6.272804 & 3.905083 \\
\hline $\mathrm{H} 77$ & -0.059560 & 5.071331 & 3.300326 \\
\hline $\mathrm{H} 78$ & -0.744722 & 4.608841 & 4.848606 \\
\hline H79 & -2.012572 & 6.599809 & 2.888147 \\
\hline H80 & -2.707908 & 6.132373 & 4.430616 \\
\hline $\mathrm{C} 81$ & -1.714504 & 2.421899 & -7.425173 \\
\hline $\mathrm{C} 82$ & -1.381341 & 3.731893 & -8.154494 \\
\hline H83 & -1.468580 & 2.514809 & -6.360139 \\
\hline H84 & -2.801180 & 2.264595 & -7.467042 \\
\hline H85 & -0.308994 & 3.950858 & -8.061717 \\
\hline H86 & -1.570635 & 3.592894 & -9.228006 \\
\hline $\mathrm{C} 87$ & 11.831545 & 2.684130 & -6.985557 \\
\hline C88 & 11.749492 & 1.740872 & -8.195285 \\
\hline H89 & 12.726134 & 2.454859 & -6.391260 \\
\hline
\end{tabular}

\begin{tabular}{|c|c|c|c|}
\hline H90 & 11.966730 & 3.711085 & -7.352230 \\
\hline H91 & 11.707666 & 0.697285 & -7.855522 \\
\hline H92 & 10.802619 & 1.927556 & -8.720796 \\
\hline C93 & 7.526998 & 0.365903 & 8.905141 \\
\hline C94 & 6.974640 & -0.644178 & 9.917882 \\
\hline H95 & 7.380343 & 1.384924 & 9.290846 \\
\hline H96 & 8.614318 & 0.231059 & 8.814407 \\
\hline H97 & 5.886932 & -0.511010 & 10.007647 \\
\hline H98 & 7.122650 & -1.663229 & 9.532202 \\
\hline C99 & 12.907004 & 1.889979 & -9.196359 \\
\hline $\mathrm{C} 100$ & 14.279495 & 1.485646 & -8.645743 \\
\hline H101 & 12.946860 & 2.929983 & -9.548727 \\
\hline H102 & 12.685543 & 1.279407 & -10.081041 \\
\hline H103 & 15.051208 & 1.559048 & -9.418969 \\
\hline H104 & 14.590288 & 2.123558 & -7.812170 \\
\hline H105 & 14.269282 & 0.450653 & -8.284414 \\
\hline C106 & 7.613518 & -0.534234 & 11.307841 \\
\hline C107 & 7.057001 & -1.548639 & 12.311216 \\
\hline H108 & 7.464613 & 0.483096 & 11.694868 \\
\hline H109 & 8.700320 & -0.666953 & 11.217459 \\
\hline H1 10 & 7.531887 & -1.444423 & 13.292139 \\
\hline H111 & 5.977590 & -1.418592 & 12.448901 \\
\hline H112 & 7.223320 & -2.576476 & 11.969010 \\
\hline $\mathrm{C} 113$ & -0.952497 & 7.380720 & 4.601703 \\
\hline $\mathrm{C} 114$ & -1.699611 & 8.716110 & 4.667679 \\
\hline H115 & 0.002741 & 7.520826 & 4.077687 \\
\hline H116 & -0.694347 & 7.054903 & 5.618514 \\
\hline H117 & -2.642878 & 8.615829 & 5.216685 \\
\hline H118 & -1.103389 & 9.485345 & 5.169280 \\
\hline H119 & -1.941085 & 9.085158 & 3.664330 \\
\hline C120 & -2.179672 & 4.951810 & -7.665313 \\
\hline C121 & -1.868484 & 5.379220 & -6.226258 \\
\hline H122 & -3.253965 & 4.741347 & -7.759982 \\
\hline H123 & -1.976778 & 5.795224 & -8.337616 \\
\hline H124 & -2.425044 & 6.282111 & -5.954531 \\
\hline H125 & -2.132489 & 4.602242 & -5.501707 \\
\hline H126 & -0.801442 & 5.597591 & -6.102201 \\
\hline
\end{tabular}

Table S8. The Cartesian Coordinates (in $\AA$ ) of $\mathrm{C}_{8}-2 \mathrm{TTN}^{\circ+}$ Optimized with the UB3LYP/6-31G(d,p) Method (The sum of electronic and zero-point energies is -3541.43708201 Hartree.)

\begin{tabular}{cccc}
\hline & $\mathrm{X}$ & $\mathrm{Y}$ & $\mathrm{Z}$ \\
\hline $\mathrm{C} 1$ & 0.000000 & 0.000000 & 0.000000 \\
$\mathrm{C} 2$ & 0.000000 & 0.000000 & 1.380000 \\
$\mathrm{C} 3$ & 5.444116 & 0.000000 & -1.715479 \\
$\mathrm{C} 4$ & 0.762862 & -0.010919 & -3.066825 \\
$\mathrm{C} 5$ & 1.405573 & -0.020290 & -4.284935 \\
$\mathrm{C} 6$ & 7.771614 & -0.003130 & -2.699588 \\
$\mathrm{C} 7$ & 6.362219 & -0.076937 & 2.964496 \\
$\mathrm{C} 8$ & 4.768764 & -0.029688 & 1.003267
\end{tabular}




\begin{tabular}{|c|c|c|c|c|c|c|c|}
\hline $\mathrm{C} 9$ & 6.473712 & -0.016688 & -0.754769 & H60 & 10.315269 & 0.840483 & -5.899647 \\
\hline $\mathrm{C} 10$ & 6.127350 & -0.034491 & 0.638155 & H61 & 10.031562 & 3.335262 & -6.045418 \\
\hline $\mathrm{C} 11$ & 1.643215 & -0.005111 & -1.958155 & H62 & 11.053526 & 3.534358 & -4.631356 \\
\hline $\mathrm{C} 12$ & 1.296039 & -0.001357 & -0.565149 & C63 & 6.971911 & -0.073565 & 4.332805 \\
\hline $\mathrm{C} 13$ & 3.001272 & -0.006178 & -2.324112 & C64 & 6.899063 & 1.304846 & 5.035270 \\
\hline $\mathrm{C} 14$ & 2.326368 & -0.004241 & 0.395649 & H65 & 8.020719 & -0.376245 & 4.234815 \\
\hline $\mathrm{C} 15$ & 4.064173 & -0.005675 & -1.380975 & H66 & 6.482152 & -0.831088 & 4.954428 \\
\hline $\mathrm{C} 16$ & 3.705881 & -0.013478 & 0.060381 & C67 & 7.570598 & 1.321159 & 6.417489 \\
\hline S17 & 3.137471 & -0.014753 & -4.081620 & H68 & 7.374457 & 2.050001 & 4.386888 \\
\hline S18 & 1.619101 & -0.000848 & 2.009309 & H69 & 5.847465 & 1.603844 & 5.132572 \\
\hline S19 & 6.148703 & 0.015222 & -3.330971 & $\mathrm{C} 70$ & 6.877163 & 0.461048 & 7.482694 \\
\hline S20 & 4.631597 & -0.060108 & 2.759518 & H71 & 8.619504 & 1.009094 & 6.316437 \\
\hline $\mathrm{C} 21$ & 7.770340 & -0.018930 & -1.321179 & $\mathrm{H} 72$ & 7.599413 & 2.361613 & 6.765212 \\
\hline $\mathrm{C} 22$ & 7.007142 & -0.060025 & 1.747185 & $\mathrm{H} 73$ & 5.821147 & 0.758924 & 7.554945 \\
\hline $\mathrm{H} 23$ & -0.914620 & -0.003352 & -0.580888 & H74 & 6.875969 & -0.594463 & 7.177314 \\
\hline $\mathrm{H} 24$ & -0.317549 & -0.015027 & -2.985164 & C75 & -1.419360 & 5.070034 & 3.240073 \\
\hline $\mathrm{H} 25$ & 8.686294 & -0.036038 & -0.742639 & C76 & -2.276334 & 6.340708 & 3.189018 \\
\hline $\mathrm{H} 26$ & 8.087555 & -0.070862 & 1.666166 & $\mathrm{H} 77$ & -0.500356 & 5.228274 & 2.657585 \\
\hline $\mathrm{C} 27$ & -1.187331 & 0.009740 & 2.300356 & H78 & -1.094819 & 4.892180 & 4.275100 \\
\hline $\mathrm{C} 28$ & -2.016492 & 1.315114 & 2.240711 & H79 & -2.598938 & 6.520090 & 2.153331 \\
\hline $\mathrm{H} 29$ & -0.862199 & -0.170683 & 3.330501 & $\mathrm{H} 80$ & -3.196708 & 6.181938 & 3.768625 \\
\hline $\mathrm{H} 30$ & -1.831360 & -0.834769 & 2.025290 & $\mathrm{C} 81$ & -1.739233 & 2.434524 & -7.295420 \\
\hline $\mathrm{C} 31$ & -1.280034 & 2.555732 & 2.756589 & C82 & -1.415439 & 3.762184 & -7.996683 \\
\hline H32 & -2.351498 & 1.482480 & 1.208972 & H83 & -1.469526 & 2.497975 & -6.232850 \\
\hline H33 & -2.924261 & 1.153149 & 2.834406 & H84 & -2.826714 & 2.282872 & -7.316118 \\
\hline $\mathrm{C} 34$ & -2.142928 & 3.823622 & 2.716118 & H85 & -0.339991 & 3.974938 & -7.922451 \\
\hline H35 & -0.946292 & 2.375095 & 3.788310 & H86 & -1.628970 & 3.651396 & -9.068417 \\
\hline H36 & -0.368916 & 2.719767 & 2.165083 & C87 & 12.133086 & 2.963217 & -6.411910 \\
\hline H37 & -2.474838 & 4.002852 & 1.683668 & C88 & 11.969899 & 2.279953 & -7.777971 \\
\hline H38 & -3.056853 & 3.659613 & 3.303658 & H89 & 12.985578 & 2.528975 & -5.873324 \\
\hline C39 & 0.785983 & -0.031406 & -5.649298 & H90 & 12.385899 & 4.019054 & -6.576588 \\
\hline $\mathrm{C} 40$ & 1.111572 & 1.215558 & -6.503767 & H91 & 11.799751 & 1.202865 & -7.642723 \\
\hline $\mathrm{H} 41$ & -0.296106 & -0.125527 & -5.515373 & H92 & 11.064769 & 2.673880 & -8.261046 \\
\hline $\mathrm{H} 42$ & 1.112518 & -0.929081 & -6.191347 & C93 & 7.530093 & 0.570466 & 8.867036 \\
\hline $\mathrm{C} 43$ & 0.467391 & 1.180275 & -7.899695 & C94 & 6.838116 & -0.284042 & 9.935676 \\
\hline $\mathrm{H} 44$ & 0.799100 & 2.113461 & -5.958270 & H95 & 7.530287 & 1.622299 & 9.185202 \\
\hline $\mathrm{H} 45$ & 2.199839 & 1.291316 & -6.619280 & H96 & 8.586493 & 0.276182 & 8.793308 \\
\hline $\mathrm{C} 46$ & -1.072417 & 1.202150 & -7.931921 & H97 & 5.782651 & 0.014342 & 10.012237 \\
\hline $\mathrm{H} 47$ & 0.855365 & 2.031665 & -8.470316 & H98 & 6.832549 & -1.335278 & 9.613200 \\
\hline $\mathrm{H} 48$ & 0.817239 & 0.283282 & -8.427949 & C99 & 13.162721 & 2.474926 & -8.728755 \\
\hline H49 & -1.387039 & 1.133698 & -8.981127 & $\mathrm{C} 100$ & 14.463535 & 1.812291 & -8.261231 \\
\hline $\mathrm{H} 50$ & -1.464373 & 0.296122 & -7.450978 & H101 & 13.332349 & 3.549976 & -8.877741 \\
\hline C51 & 8.953190 & 0.043209 & -3.619715 & H102 & 12.892644 & 2.072744 & -9.713101 \\
\hline $\mathrm{C} 52$ & 9.303970 & 1.471659 & -4.102517 & H103 & 15.255844 & 1.936646 & -9.005874 \\
\hline H53 & 8.773041 & -0.597765 & -4.490676 & H104 & 14.828969 & 2.240853 & -7.322413 \\
\hline $\mathrm{H} 54$ & 9.812432 & -0.382154 & -3.088898 & H105 & 14.323983 & 0.736353 & -8.103193 \\
\hline $\mathrm{C} 55$ & 10.519928 & 1.486087 & -5.036294 & C106 & 7.492786 & -0.184530 & 11.319167 \\
\hline H56 & 8.435069 & 1.901827 & -4.616572 & C107 & 6.793414 & -1.038742 & 12.380336 \\
\hline H57 & 9.493135 & 2.107617 & -3.228788 & H108 & 7.500517 & 0.865650 & 11.640608 \\
\hline C58 & 10.887989 & 2.899049 & -5.511834 & H109 & 8.546324 & -0.485544 & 11.242152 \\
\hline H59 & 11.382197 & 1.042186 & -4.518911 & H1 10 & 7.287291 & -0.949850 & 13.352795 \\
\hline
\end{tabular}




\begin{tabular}{rrrr}
$\mathrm{H} 111$ & 5.748536 & -0.734692 & 12.509876 \\
$\mathrm{H} 112$ & 6.796582 & -2.098723 & 12.102077 \\
$\mathrm{C} 113$ & -1.555573 & 7.587156 & 3.716744 \\
$\mathrm{C} 114$ & -2.415809 & 8.852703 & 3.657003 \\
$\mathrm{H} 115$ & -0.633532 & 7.742956 & 3.139935 \\
$\mathrm{H} 116$ & -1.237715 & 7.408637 & 4.752987 \\
$\mathrm{H} 117$ & -3.330174 & 8.739068 & 4.249947 \\
$\mathrm{H} 118$ & -1.875248 & 9.721851 & 4.044413 \\
$\mathrm{H} 119$ & -2.716372 & 9.079986 & 2.628032 \\
$\mathrm{C} 120$ & -2.197846 & 4.971522 & -7.458580 \\
$\mathrm{C} 121$ & -1.858245 & 5.358924 & -6.014844 \\
$\mathrm{H} 122$ & -3.274386 & 4.769675 & -7.539707 \\
$\mathrm{H} 123$ & -2.002176 & 5.831008 & -8.111243 \\
$\mathrm{H} 124$ & -2.403077 & 6.258370 & -5.711391 \\
$\mathrm{H} 125$ & -2.117686 & 4.566508 & -5.304599 \\
$\mathrm{H} 126$ & -0.787492 & 5.567965 & -5.903840 \\
\hline
\end{tabular}

Table S9. The Cartesian Coordinates (in $\AA$ ) of $\mathrm{C}_{10}-2 \mathrm{TTN}$ Optimized with the B3LYP/6-31G(d,p) Method (The sum of electronic and zero-point energies is -3856.19692411 Hartree.)

\begin{tabular}{rcrr} 
Hartree. & & & \\
\hline & $\mathrm{X}$ & $\mathrm{Y}$ & $\mathrm{Z}$ \\
\hline $\mathrm{C} 1$ & 0.000000 & 0.000000 & 0.000000 \\
$\mathrm{C} 2$ & 0.000000 & 0.000000 & 1.362000 \\
$\mathrm{C} 3$ & 5.463464 & 0.000000 & -1.715288 \\
$\mathrm{C} 4$ & 0.767070 & -0.010979 & -3.088346 \\
$\mathrm{C} 5$ & 1.403819 & -0.017061 & -4.292548 \\
$\mathrm{C} 6$ & 7.799819 & 0.011199 & -2.704328 \\
$\mathrm{C} 7$ & 6.394797 & -0.067701 & 2.950079 \\
$\mathrm{C} 8$ & 4.793471 & -0.020935 & 0.982606 \\
$\mathrm{C} 9$ & 6.490330 & -0.010204 & -0.761094 \\
$\mathrm{C} 10$ & 6.147543 & -0.026678 & 0.619978 \\
$\mathrm{C} 11$ & 1.653260 & -0.002924 & -1.961868 \\
$\mathrm{C} 12$ & 1.310257 & -0.000563 & -0.580828 \\
$\mathrm{C} 13$ & 3.007062 & -0.002750 & -2.325162 \\
$\mathrm{C} 14$ & 2.337089 & -0.002564 & 0.373308 \\
$\mathrm{C} 15$ & 4.074396 & -0.002293 & -1.372579 \\
$\mathrm{C} 16$ & 3.726041 & -0.006566 & 0.030238 \\
$\mathrm{~S} 17$ & 3.149188 & -0.008716 & -4.082360 \\
$\mathrm{~S} 18$ & 1.640624 & -0.000859 & 1.992762 \\
$\mathrm{~S} 19$ & 6.159024 & 0.016215 & -3.334628 \\
$\mathrm{~S} 20$ & 4.649946 & -0.045098 & 2.739143 \\
$\mathrm{C} 21$ & 7.800188 & -0.003794 & -1.342411 \\
$\mathrm{C} 22$ & 7.032779 & -0.053554 & 1.746824 \\
$\mathrm{H} 23$ & -0.914364 & 0.003511 & -0.582967 \\
$\mathrm{H} 24$ & -0.313804 & -0.010939 & -3.000973 \\
$\mathrm{H} 25$ & 8.714715 & -0.011363 & -0.759740 \\
$\mathrm{H} 26$ & 8.113646 & -0.063460 & 1.659919 \\
$\mathrm{C} 27$ & -1.178060 & -0.020974 & 2.293525 \\
$\mathrm{C} 28$ & -1.339757 & -1.338443 & 3.078707 \\
$\mathrm{H} 29$ & -2.079114 & 0.159583 & 1.694618 \\
& & &
\end{tabular}

\begin{tabular}{|c|c|c|c|}
\hline $\mathrm{H} 30$ & -1.109381 & 0.813686 & 3.004389 \\
\hline C31 & -2.578409 & -1.336944 & 3.984314 \\
\hline H32 & -1.402903 & -2.171183 & 2.366162 \\
\hline H33 & -0.435175 & -1.512192 & 3.673011 \\
\hline C34 & -2.809721 & -2.649212 & 4.751156 \\
\hline H35 & -2.504215 & -0.506397 & 4.701117 \\
\hline H36 & -3.464063 & -1.126427 & 3.369138 \\
\hline H37 & -3.794409 & -2.602568 & 5.235611 \\
\hline H38 & -2.865566 & -3.481590 & 4.035120 \\
\hline C39 & 0.803136 & -0.041949 & -5.670071 \\
\hline $\mathrm{C} 40$ & 1.017507 & -1.359932 & -6.448432 \\
\hline $\mathrm{H} 41$ & 1.206749 & 0.788758 & -6.262969 \\
\hline $\mathrm{H} 42$ & -0.272483 & 0.146878 & -5.566163 \\
\hline $\mathrm{C} 43$ & 0.348205 & -2.575809 & -5.796045 \\
\hline H44 & 0.631305 & -1.214817 & -7.464248 \\
\hline $\mathrm{H} 45$ & 2.094670 & -1.547301 & -6.551662 \\
\hline $\mathrm{C} 46$ & 0.606566 & -3.909478 & -6.515675 \\
\hline $\mathrm{H} 47$ & 0.700478 & -2.659387 & -4.760936 \\
\hline $\mathrm{H} 48$ & -0.736044 & -2.399937 & -5.735274 \\
\hline H49 & 1.690580 & -4.082753 & -6.575414 \\
\hline $\mathrm{H} 50$ & 0.206724 & -4.723352 & -5.895821 \\
\hline C51 & 8.975984 & 0.046183 & -3.637824 \\
\hline C52 & 9.138615 & 1.378311 & -4.397874 \\
\hline H53 & 8.903292 & -0.774989 & -4.363837 \\
\hline H54 & 9.878239 & -0.147566 & -3.044876 \\
\hline C55 & 10.361183 & 1.384123 & -5.325226 \\
\hline H56 & 8.233029 & 1.571607 & -4.987734 \\
\hline H57 & 9.206297 & 2.191517 & -3.666060 \\
\hline C58 & 10.519581 & 2.663475 & -6.162942 \\
\hline H59 & 11.271575 & 1.225876 & -4.729185 \\
\hline $\mathrm{H} 60$ & 10.291738 & 0.523973 & -6.004947 \\
\hline H61 & 11.325371 & 2.507022 & -6.892810 \\
\hline H62 & 9.605590 & 2.824017 & -6.752457 \\
\hline $\mathrm{C} 63$ & 6.995457 & -0.128787 & 4.325499 \\
\hline C64 & 6.735489 & -1.457824 & 5.063791 \\
\hline $\mathrm{H} 65$ & 6.619568 & 0.702136 & 4.937853 \\
\hline H66 & 8.076461 & 0.027649 & 4.224770 \\
\hline C67 & 7.393473 & -1.507051 & 6.449077 \\
\hline H68 & 5.652688 & -1.606535 & 5.168815 \\
\hline H69 & 7.097632 & -2.280373 & 4.436685 \\
\hline $\mathrm{C} 70$ & 7.074934 & -2.774341 & 7.259084 \\
\hline H71 & 8.483242 & -1.410460 & 6.338649 \\
\hline $\mathrm{H} 72$ & 7.069215 & -0.629933 & 7.025846 \\
\hline $\mathrm{H} 73$ & 7.475300 & -2.651617 & 8.274529 \\
\hline H74 & 5.985162 & -2.863711 & 7.373498 \\
\hline C75 & -1.752966 & -2.966738 & 5.818530 \\
\hline C76 & -2.056300 & -4.257101 & 6.593189 \\
\hline H77 & -1.685438 & -2.118503 & 6.512779 \\
\hline $\mathrm{H} 78$ & -0.762866 & -3.059348 & 5.352517 \\
\hline H79 & -3.034736 & -4.163557 & 7.086171 \\
\hline $\mathrm{H} 80$ & -2.159277 & -5.081076 & 5.874048 \\
\hline
\end{tabular}




\begin{tabular}{|c|c|c|c|c|c|c|c|}
\hline $\mathrm{C} 81$ & -0.002299 & -4.018827 & -7.920725 & H132 & 7.807329 & -7.131497 & 9.742840 \\
\hline $\mathrm{C} 82$ & 0.217291 & -5.398545 & -8.557843 & H133 & 9.243511 & -7.857659 & 9.044222 \\
\hline H83 & 0.419647 & -3.241824 & -8.569554 & H134 & 8.198263 & 10.147571 & 9.274216 \\
\hline H84 & -1.082104 & -3.815928 & -7.863333 & H135 & 8.282160 & -9.324239 & 10.837555 \\
\hline H85 & 1.296705 & -5.589485 & -8.643334 & H136 & 6.747043 & -9.412759 & 9.964428 \\
\hline H86 & -0.170188 & -6.165880 & -7.873754 & $\mathrm{C} 137$ & 0.126431 & -4.144461 & 9.887135 \\
\hline $\mathrm{C} 87$ & 10.829279 & 3.929710 & -5.352600 & C138 & 0.227268 & -3.193375 & 11.083280 \\
\hline C88 & 11.034720 & 5.173449 & -6.226356 & H139 & 1.113538 & -4.245231 & 9.415557 \\
\hline H89 & 11.731104 & 3.758381 & -4.746863 & H140 & -0.143505 & -5.149408 & 10.239466 \\
\hline H90 & 10.017907 & 4.126645 & -4.639665 & H141 & 0.961013 & -3.545535 & 11.815678 \\
\hline H91 & 11.848221 & 4.985720 & -6.941810 & H142 & 0.529613 & -2.188691 & 10.766401 \\
\hline H92 & 10.133139 & 5.342789 & -6.832290 & H143 & -0.736167 & -3.099814 & 11.597338 \\
\hline $\mathrm{C} 93$ & 7.629378 & -4.076623 & 6.665307 & C144 & -0.489236 & -4.990869 & -12.426281 \\
\hline C94 & 7.337527 & -5.308167 & 7.532248 & $\mathrm{C} 145$ & 0.088387 & -4.117500 & -13.543899 \\
\hline H95 & 8.716074 & -3.977006 & 6.528457 & H146 & -1.574805 & -4.834260 & -12.363542 \\
\hline H96 & 7.211154 & -4.240024 & 5.663422 & H147 & -0.351454 & -6.050570 & -12.680744 \\
\hline H97 & 7.753919 & -5.146707 & 8.535319 & H148 & -0.381331 & -4.336694 & -14.508329 \\
\hline H98 & 6.250403 & -5.406044 & 7.666937 & H149 & -0.065017 & -3.052722 & -13.333782 \\
\hline C99 & 11.349069 & 6.440962 & -5.422479 & H150 & 1.166765 & -4.278388 & -13.655399 \\
\hline $\mathrm{C} 100$ & 11.540654 & 7.689241 & -6.292197 & \multirow{5}{*}{\multicolumn{4}{|c|}{$\begin{array}{l}\text { Table S10. The Cartesian Coordinates (in } \AA \text { ) of } \mathrm{C}_{10}-\mathrm{TTN}^{*} \\
\text { Optimized with the UB3LYP/6-31G(d,p) Method (The sum } \\
\text { of electronic and zero-point energies is }-3855.96964123 \\
\text { Hartree.) }\end{array}$}} \\
\hline H101 & 12.255195 & 6.274436 & -4.822391 & & & & \\
\hline H102 & 10.539081 & 6.622532 & -4.701624 & & & & \\
\hline H103 & 12.349171 & 7.509080 & -7.015359 & & & & \\
\hline H104 & 10.633598 & 7.857942 & -6.890268 & & & & \\
\hline C105 & 7.889326 & -6.611548 & 6.937903 & & $X$ & $\mathrm{Y}$ & Z \\
\hline C106 & 7.532247 & -7.882023 & 7.725980 & $\mathrm{C} 1$ & 0.000000 & 0.000000 & 0.000000 \\
\hline H107 & 8.982737 & -6.535257 & 6.849854 & $\mathrm{C} 2$ & 0.000000 & 0.000000 & 1.378100 \\
\hline H108 & 7.511450 & -6.716724 & 5.911818 & $\mathrm{C} 3$ & 5.440923 & 0.000000 & -1.724917 \\
\hline H109 & 6.438589 & -7.960234 & 7.810449 & $\mathrm{C} 4$ & 0.757935 & -0.004101 & -3.069609 \\
\hline H110 & 7.852062 & -8.758128 & 7.145205 & $\mathrm{C} 5$ & 1.399642 & -0.003993 & -4.288283 \\
\hline C111 & -0.997926 & -4.645325 & 7.638524 & C6 & 7.766454 & 0.013622 & -2.713065 \\
\hline $\mathrm{C} 112$ & -0.891390 & -3.688564 & 8.833877 & $\mathrm{C} 7$ & 6.367183 & -0.082729 & 2.953453 \\
\hline H113 & -0.017074 & -4.724080 & 7.147986 & $\mathrm{C} 8$ & 4.769943 & -0.036130 & 0.995289 \\
\hline H114 & -1.227802 & -5.651566 & 8.014473 & C9 & 6.472271 & -0.015833 & -0.765927 \\
\hline H115 & -0.618468 & -2.682703 & 8.488072 & $\mathrm{C} 10$ & 6.127833 & -0.039528 & 0.627582 \\
\hline H116 & -1.880094 & -3.589087 & 9.305416 & $\mathrm{C} 11$ & 1.640124 & -0.003782 & -1.961744 \\
\hline C117 & -0.440505 & -5.589107 & -9.934172 & $\mathrm{C} 12$ & 1.295845 & -0.004750 & -0.568570 \\
\hline $\mathrm{C} 118$ & 0.139831 & -4.715506 & -11.054452 & $\mathrm{C} 13$ & 2.997579 & -0.003293 & -2.329543 \\
\hline H119 & -1.520113 & -5.398601 & -9.848886 & $\mathrm{C} 14$ & 2.326432 & -0.011008 & 0.390466 \\
\hline H120 & -0.343656 & -6.643735 & -10.226236 & $\mathrm{C} 15$ & 4.061705 & -0.006380 & -1.388457 \\
\hline H121 & 0.007122 & -3.653356 & -10.809847 & $\mathrm{C} 16$ & 3.705686 & -0.017155 & 0.053215 \\
\hline H122 & 1.225797 & -4.879179 & -11.115023 & S17 & 3.131384 & -0.002310 & -4.087007 \\
\hline C123 & 11.857120 & 8.955692 & -5.487286 & S18 & 1.624944 & -0.007941 & 2.008056 \\
\hline C124 & 12.042058 & 10.199055 & -6.362122 & S19 & 6.142728 & 0.023315 & -3.341710 \\
\hline H125 & 12.765418 & 8.788161 & -4.892433 & S20 & 4.636437 & -0.065328 & 2.752150 \\
\hline H126 & 11.050592 & 9.134001 & -4.763117 & $\mathrm{C} 21$ & 7.767733 & -0.008894 & -1.334449 \\
\hline H127 & 11.137462 & 10.413039 & -6.942754 & $\mathrm{C} 22$ & 7.009817 & -0.065388 & 1.734825 \\
\hline H128 & 12.265897 & 11.084717 & -5.758544 & $\mathrm{H} 23$ & -0.917023 & 0.007202 & -0.577051 \\
\hline H129 & 12.865017 & 10.064616 & -7.073374 & $\mathrm{H} 24$ & -0.322285 & -0.002386 & -2.986678 \\
\hline $\mathrm{C} 130$ & 8.153539 & -7.970513 & 9.126723 & $\mathrm{H} 25$ & 8.685099 & -0.020903 & -0.757834 \\
\hline $\mathrm{C} 131$ & 7.828726 & -9.285816 & 9.841700 & $\mathrm{H} 26$ & 8.090135 & -0.070907 & 1.652020 \\
\hline
\end{tabular}




\begin{tabular}{|c|c|c|c|c|c|c|c|}
\hline $\mathrm{C} 27$ & -1.182650 & -0.025599 & 2.297658 & $\mathrm{H} 78$ & -0.853616 & -3.171066 & 5.261071 \\
\hline $\mathrm{C} 28$ & -1.373344 & -1.374533 & 3.029768 & H79 & -3.176601 & -4.300839 & 6.905915 \\
\hline $\mathrm{H} 29$ & -2.074371 & 0.198952 & 1.701681 & H80 & -2.283311 & -5.189648 & 5.685594 \\
\hline H30 & -1.091896 & 0.777093 & 3.040300 & $\mathrm{C} 81$ & 0.047327 & -3.904083 & -8.045162 \\
\hline C31 & -2.627204 & -1.379563 & 3.914705 & C82 & 0.269770 & -5.257584 & -8.735761 \\
\hline H32 & -1.440773 & -2.178547 & 2.285926 & H83 & 0.467304 & -3.102665 & -8.664921 \\
\hline H33 & -0.481472 & -1.582533 & 3.631862 & H84 & -1.032539 & -3.706130 & -7.978201 \\
\hline C34 & -2.885025 & -2.714520 & 4.632781 & H85 & 1.349611 & -5.447193 & -8.818790 \\
\hline H35 & -2.549738 & -0.574382 & 4.658670 & H86 & -0.126610 & -6.050397 & -8.087060 \\
\hline H36 & -3.498244 & -1.136308 & 3.291857 & $\mathrm{C} 87$ & 11.317441 & 3.955246 & -4.485345 \\
\hline H37 & -3.877510 & -2.667561 & 5.098908 & C88 & 11.737404 & 5.313462 & -5.061838 \\
\hline H38 & -2.940437 & -3.521679 & 3.888694 & H89 & 12.165186 & 3.514547 & -3.941071 \\
\hline C39 & 0.787909 & -0.010044 & -5.657752 & H90 & 10.527527 & 4.116524 & -3.738909 \\
\hline $\mathrm{C} 40$ & 1.032816 & -1.303922 & -6.471512 & H91 & 12.534742 & 5.160924 & -5.802638 \\
\hline H41 & 1.172902 & 0.846046 & -6.225466 & H92 & 10.892564 & 5.750264 & -5.613118 \\
\hline $\mathrm{H} 42$ & -0.289582 & 0.151151 & -5.540249 & $\mathrm{C} 93$ & 7.991688 & -4.132789 & 6.453538 \\
\hline $\mathrm{C} 43$ & 0.388450 & -2.553308 & -5.858522 & C94 & 7.825056 & -5.427805 & 7.259295 \\
\hline $\mathrm{H} 44$ & 0.639942 & -1.130465 & -7.478670 & H95 & 9.062780 & -3.922097 & 6.321286 \\
\hline $\mathrm{H} 45$ & 2.113581 & -1.461266 & -6.586544 & H96 & 7.585831 & -4.289456 & 5.444748 \\
\hline $\mathrm{C} 46$ & 0.658804 & -3.851060 & -6.637994 & H97 & 8.228303 & -5.275074 & 8.268538 \\
\hline $\mathrm{H} 47$ & 0.752463 & -2.677952 & -4.830143 & H98 & 6.753348 & -5.637669 & 7.388676 \\
\hline $\mathrm{H} 48$ & -0.696863 & -2.395078 & -5.781321 & C99 & 12.216661 & 6.307587 & -3.997108 \\
\hline H49 & 1.744252 & -4.011847 & -6.706051 & $\mathrm{C} 100$ & 12.636455 & 7.666461 & -4.570302 \\
\hline H50 & 0.267528 & -4.692603 & -6.051586 & H101 & 13.061255 & 5.869542 & -3.446237 \\
\hline C51 & 8.946063 & 0.082132 & -3.634332 & H102 & 11.418334 & 6.458555 & -3.256013 \\
\hline C52 & 9.271483 & 1.521108 & -4.103772 & H103 & 13.434363 & 7.517267 & -5.311614 \\
\hline H53 & 8.775746 & -0.555319 & -4.509970 & H104 & 11.791932 & 8.105532 & -5.120463 \\
\hline H54 & 9.812608 & -0.332604 & -3.106990 & $\mathrm{C} 105$ & 8.499738 & -6.639188 & 6.600595 \\
\hline C55 & 10.492962 & 1.561936 & -5.032009 & $\mathrm{C} 106$ & 8.271276 & -7.976344 & 7.323221 \\
\hline H56 & 8.398447 & 1.937139 & -4.623071 & H107 & 9.580015 & -6.451420 & 6.519085 \\
\hline H57 & 9.437375 & 2.149242 & -3.221702 & H108 & 8.130615 & -6.728934 & 5.569442 \\
\hline C58 & 10.838325 & 2.965784 & -5.556481 & H109 & 7.191079 & -8.169399 & 7.395634 \\
\hline H59 & 11.363498 & 1.147424 & -4.504403 & H110 & 8.679402 & -8.784235 & 6.700945 \\
\hline H60 & 10.308532 & 0.897238 & -5.886273 & C111 & -1.159129 & -4.823020 & 7.490686 \\
\hline H61 & 11.622128 & 2.863248 & -6.317825 & $\mathrm{C} 112$ & -1.075130 & -3.909347 & 8.721034 \\
\hline H62 & 9.965411 & 3.383365 & -6.077700 & H113 & -0.166802 & -4.895844 & 7.021897 \\
\hline C63 & 6.980545 & -0.152582 & 4.318996 & H114 & -1.407867 & -5.838930 & 7.824841 \\
\hline C64 & 6.831074 & -1.536983 & 4.992787 & H115 & -0.785776 & -2.893437 & 8.419167 \\
\hline H65 & 6.536909 & 0.614737 & 4.965554 & H116 & -2.074273 & -3.818202 & 9.170818 \\
\hline H66 & 8.043534 & 0.095427 & 4.222440 & C117 & -0.374912 & -5.386983 & -10.125341 \\
\hline C67 & 7.507869 & -1.588643 & 6.369114 & $\mathrm{C} 118$ & 0.223183 & -4.473301 & -11.203518 \\
\hline H68 & 5.764195 & -1.773527 & 5.098619 & H119 & -1.454325 & -5.195052 & -10.043424 \\
\hline H69 & 7.253402 & -2.298483 & 4.327884 & H120 & -0.279954 & -6.429457 & -10.456753 \\
\hline $\mathrm{C} 70$ & 7.317756 & -2.921180 & 7.112360 & H121 & 0.091402 & -3.419991 & -10.921284 \\
\hline H71 & 8.581507 & -1.385196 & 6.252962 & H122 & 1.309078 & -4.639845 & -11.257124 \\
\hline $\mathrm{H} 72$ & 7.108794 & -0.775361 & 6.989885 & $\mathrm{C} 123$ & 13.116216 & 8.659489 & -3.504631 \\
\hline $\mathrm{H} 73$ & 7.713585 & -2.807061 & 8.129613 & $\mathrm{C} 124$ & 13.532987 & 10.014253 & -4.084373 \\
\hline H74 & 6.242761 & -3.119452 & 7.228698 & H125 & 13.960473 & 8.220369 & -2.955899 \\
\hline $\mathrm{C} 75$ & -1.852937 & -3.079373 & 5.709125 & H126 & 12.318525 & 8.807886 & -2.763814 \\
\hline $\mathrm{C} 76$ & -2.188233 & -4.391005 & 6.433665 & H127 & 12.699355 & 10.494511 & -4.609164 \\
\hline $\mathrm{H} 77$ & -1.789529 & -2.256273 & 6.433065 & H128 & 13.870118 & 10.698686 & -3.299489 \\
\hline
\end{tabular}




\begin{tabular}{rrrr} 
H129 & 14.353315 & 9.902806 & -4.802301 \\
C130 & 8.896109 & -8.072283 & 8.722007 \\
C131 & 8.706961 & -9.449159 & 9.365855 \\
H132 & 8.464045 & -7.306421 & 9.378395 \\
H133 & 9.968762 & -7.844195 & 8.653061 \\
H134 & 9.165157 & -10.237634 & 8.758284 \\
H135 & 9.160356 & -9.491087 & 10.361146 \\
H136 & 7.644120 & -9.693193 & 9.474546 \\
C137 & -0.087929 & -4.411192 & 9.782659 \\
C138 & -0.010237 & -3.503907 & 11.013912 \\
H139 & 0.910043 & -4.505315 & 9.332598 \\
H140 & -0.375634 & -5.424954 & 10.092135 \\
H141 & 0.701846 & -3.888685 & 11.750820 \\
H142 & 0.307866 & -2.490812 & 10.741690 \\
H143 & -0.985242 & -3.420947 & 11.506905 \\
C144 & -0.390161 & -4.693584 & -12.592422 \\
C145 & 0.205651 & -3.781689 & -13.668682 \\
H146 & -1.475591 & -4.534710 & -12.536907 \\
H147 & -0.253052 & -5.743337 & -12.884420 \\
H148 & -0.252985 & -3.963344 & -14.645711 \\
H149 & 0.053454 & -2.724605 & -13.422016 \\
H150 & 1.284429 & -3.943758 & -13.773416 \\
\hline
\end{tabular}

Table S11. The Cartesian Coordinates (in $\AA$ ) of DNTT Optimized with the B3LYP/6-31G(d,p) Method (The sum of electronic and zero-point energies is -1641.741969 Hartree.)

\begin{tabular}{rrrr}
\hline & $\mathrm{X}$ & $\mathrm{Y}$ & $\mathrm{Z}$ \\
\hline $\mathrm{C} 1$ & 2.881977 & 1.182556 & 0.000088 \\
$\mathrm{H} 2$ & 2.714251 & 2.256274 & 0.000222 \\
$\mathrm{C} 3$ & 4.206032 & 0.690498 & 0.000109 \\
$\mathrm{C} 4$ & 1.807306 & 0.307516 & -0.000131 \\
$\mathrm{C} 5$ & 4.435803 & -0.731618 & -0.000023 \\
$\mathrm{C} 6$ & 5.332075 & 1.563270 & 0.000293 \\
$\mathrm{C} 7$ & 2.051801 & -1.110497 & -0.000229 \\
$\mathrm{C} 8$ & 3.330387 & -1.618715 & -0.000147 \\
$\mathrm{C} 9$ & 5.776604 & -1.208178 & 0.000029 \\
$\mathrm{C} 10$ & 6.612734 & 1.066632 & 0.000344 \\
$\mathrm{C} 11$ & 6.837784 & -0.334791 & 0.000208 \\
$\mathrm{H} 12$ & 5.156848 & 2.635902 & 0.000365 \\
$\mathrm{H} 13$ & 3.508871 & -2.690231 & -0.000212 \\
$\mathrm{H} 14$ & 5.946509 & -2.281681 & -0.000095 \\
$\mathrm{H} 15$ & 7.460111 & 1.745680 & 0.000465 \\
$\mathrm{H} 16$ & 7.855071 & -0.714638 & 0.000230 \\
$\mathrm{C} 17$ & 0.392834 & 0.562651 & -0.000008 \\
$\mathrm{~S} 18$ & 0.545125 & -2.052021 & -0.000257 \\
$\mathrm{C} 19$ & -0.392850 & -0.562451 & -0.000003 \\
$\mathrm{~S} 20$ & -0.545173 & 2.052150 & -0.000271 \\
$\mathrm{C} 21$ & -1.807293 & -0.307373 & -0.000124 \\
$\mathrm{C} 22$ & -2.051845 & 1.110643 & -0.000228 \\
$\mathrm{C} 23$ & -3.330482 & 1.618742 & -0.000145 \\
$\mathrm{C} 24$ & -4.435828 & 0.731579 & -0.000018 \\
& & & \\
\hline
\end{tabular}

\begin{tabular}{lrrr}
$\mathrm{C} 25$ & -4.205978 & -0.690529 & 0.000109 \\
$\mathrm{C} 26$ & -5.776673 & 1.208028 & 0.000040 \\
$\mathrm{C} 27$ & -2.881890 & -1.182509 & 0.000093 \\
$\mathrm{C} 28$ & -5.331957 & -1.563389 & 0.000280 \\
$\mathrm{C} 29$ & -6.837785 & 0.334564 & 0.000215 \\
$\mathrm{C} 30$ & -6.612651 & -1.066850 & 0.000332 \\
$\mathrm{H} 31$ & -5.946666 & 2.281518 & -0.000071 \\
$\mathrm{H} 32$ & -2.714086 & -2.256211 & 0.000227 \\
$\mathrm{H} 33$ & -5.156635 & -2.636005 & 0.000342 \\
$\mathrm{H} 34$ & -7.855096 & 0.714348 & 0.000246 \\
$\mathrm{H} 35$ & -7.459989 & -1.745946 & 0.000441 \\
$\mathrm{H} 36$ & -3.509054 & 2.690247 & -0.000207 \\
\hline
\end{tabular}

Table S12. The Cartesian Coordinates (in $\AA$ ) of $\mathrm{DNTT}^{\circ+}$ Optimized with the UB3LYP/6-31G(d,p) Method (The sum of electronic and zero-point energies is -1641.505795 Hartree.)

\begin{tabular}{|c|c|c|c|}
\hline & $\mathrm{X}$ & $\mathrm{Y}$ & Z \\
\hline $\mathrm{C} 1$ & 2.886117 & -1.188292 & -0.000009 \\
\hline $\mathrm{H} 2$ & 2.722847 & -2.262327 & -0.000014 \\
\hline $\mathrm{C} 3$ & 4.205888 & -0.688787 & 0.000002 \\
\hline $\mathrm{C} 4$ & 1.801514 & -0.311132 & -0.000012 \\
\hline C5 & 4.427059 & 0.734159 & 0.000011 \\
\hline C6 & 5.328312 & -1.561298 & 0.000005 \\
\hline $\mathrm{C} 7$ & 2.041095 & 1.105382 & -0.000004 \\
\hline $\mathrm{C} 8$ & 3.310860 & 1.622656 & 0.000008 \\
\hline C9 & 5.753839 & 1.218094 & 0.000021 \\
\hline $\mathrm{C} 10$ & 6.612036 & -1.055317 & 0.000016 \\
\hline $\mathrm{C} 11$ & 6.824979 & 0.339863 & 0.000024 \\
\hline H12 & 5.159746 & -2.634048 & -0.000002 \\
\hline H13 & 3.486072 & 2.694130 & 0.000011 \\
\hline H14 & 5.926177 & 2.290114 & 0.000027 \\
\hline H15 & 7.462309 & -1.729000 & 0.000018 \\
\hline H16 & 7.838620 & 0.727290 & 0.000033 \\
\hline $\mathrm{C} 17$ & 0.401920 & -0.579320 & -0.000017 \\
\hline S18 & 0.528400 & 2.039991 & -0.000021 \\
\hline C19 & -0.401920 & 0.579324 & -0.000017 \\
\hline $\mathrm{S} 20$ & -0.528399 & -2.039988 & -0.000021 \\
\hline $\mathrm{C} 21$ & -1.801514 & 0.311135 & -0.000012 \\
\hline $\mathrm{C} 22$ & -2.041093 & -1.105380 & -0.000004 \\
\hline $\mathrm{C} 23$ & -3.310857 & -1.622656 & 0.000008 \\
\hline $\mathrm{C} 24$ & -4.427059 & -0.734160 & 0.000011 \\
\hline $\mathrm{C} 25$ & -4.205890 & 0.688787 & 0.000002 \\
\hline $\mathrm{C} 26$ & -5.753838 & -1.218097 & 0.000021 \\
\hline $\mathrm{C} 27$ & -2.886118 & 1.188292 & -0.000009 \\
\hline $\mathrm{C} 28$ & -5.328315 & 1.561295 & 0.000005 \\
\hline $\mathrm{C} 29$ & -6.824979 & -0.339868 & 0.000024 \\
\hline $\mathrm{C} 30$ & -6.612038 & 1.055312 & 0.000016 \\
\hline H31 & -5.926173 & -2.290118 & 0.000027 \\
\hline H32 & -2.722850 & 2.262329 & -0.000014 \\
\hline H33 & -5.159751 & 2.634046 & -0.000002 \\
\hline
\end{tabular}




\begin{tabular}{rrrr} 
H34 & -7.838620 & -0.727297 & 0.000034 \\
H35 & -7.462312 & 1.728993 & 0.000018 \\
H36 & -3.486068 & -2.694129 & 0.000011 \\
\hline
\end{tabular}

Table S13. The Cartesian Coordinates (in $\AA$ ) of DPh-BTBT Optimized with the B3LYP/6-31G(d,p) Method (The sum of electronic and zero-point energies is -1796.511043 Hartree.)

\begin{tabular}{|c|c|c|c|}
\hline & $\mathrm{X}$ & $\mathrm{Y}$ & Z \\
\hline $\mathrm{C} 1$ & 1.689824 & 0.702571 & -0.005764 \\
\hline $\mathrm{C} 2$ & 2.243874 & -0.605090 & -0.008831 \\
\hline $\mathrm{S} 3$ & 0.999333 & -1.865985 & -0.004449 \\
\hline $\mathrm{C} 4$ & 0.257586 & 0.639072 & -0.005944 \\
\hline $\mathrm{C} 5$ & -0.257584 & -0.639073 & -0.005943 \\
\hline C6 & -1.689825 & -0.702571 & -0.005764 \\
\hline S7 & -0.999333 & 1.865985 & -0.004449 \\
\hline $\mathrm{C} 8$ & -2.243874 & 0.605089 & -0.008832 \\
\hline C9 & -3.620969 & 0.814039 & -0.004600 \\
\hline $\mathrm{C} 10$ & -4.487946 & -0.286947 & -0.001221 \\
\hline $\mathrm{C} 11$ & -2.565739 & -1.801132 & -0.001925 \\
\hline $\mathrm{C} 12$ & -3.935251 & -1.589116 & -0.000070 \\
\hline H13 & -4.022950 & 1.821673 & 0.023068 \\
\hline $\mathrm{C} 14$ & -5.959186 & -0.088473 & 0.001326 \\
\hline H15 & -2.169186 & -2.812131 & -0.010589 \\
\hline H16 & -4.604135 & -2.443174 & -0.025357 \\
\hline $\mathrm{C} 17$ & 3.620970 & -0.814039 & -0.004598 \\
\hline $\mathrm{C} 18$ & 4.487945 & 0.286947 & -0.001220 \\
\hline $\mathrm{C} 19$ & 2.565739 & 1.801132 & -0.001926 \\
\hline $\mathrm{C} 20$ & 3.935251 & 1.589117 & -0.000071 \\
\hline $\mathrm{H} 21$ & 4.022953 & -1.821672 & 0.023070 \\
\hline $\mathrm{C} 22$ & 5.959185 & 0.088473 & 0.001326 \\
\hline $\mathrm{H} 23$ & 2.169183 & 2.812131 & -0.010590 \\
\hline $\mathrm{H} 24$ & 4.604137 & 2.443173 & -0.025358 \\
\hline $\mathrm{C} 25$ & -6.549945 & 0.953378 & -0.734167 \\
\hline $\mathrm{C} 26$ & -6.800967 & -0.937261 & 0.740577 \\
\hline $\mathrm{C} 27$ & -8.182005 & -0.750246 & 0.744947 \\
\hline $\mathrm{C} 28$ & -7.930727 & 1.141959 & -0.727752 \\
\hline $\mathrm{C} 29$ & -8.753608 & 0.290724 & 0.011485 \\
\hline $\mathrm{H} 30$ & -6.365552 & -1.732812 & 1.337718 \\
\hline H31 & -8.811886 & -1.413792 & 1.330498 \\
\hline H32 & -5.922855 & 1.602955 & -1.337534 \\
\hline H33 & -8.365218 & 1.949269 & -1.310288 \\
\hline H34 & -9.829684 & 0.436561 & 0.015295 \\
\hline
\end{tabular}

Table S14. The Cartesian Coordinates (in $\AA$ ) of DPh-BTBT ${ }^{*+}$ Optimized with the UB3LYP/6-31G(d,p) Method (The sum of electronic and zero-point energies is -1796.270911 Hartree.)

\begin{tabular}{|c|c|c|c|}
\hline & $X$ & Y & Z \\
\hline $\mathrm{C} 1$ & -1.673671 & -0.710019 & -0.005982 \\
\hline $\mathrm{C} 2$ & -2.228416 & 0.605697 & -0.013925 \\
\hline S3 & -0.979580 & 1.875231 & -0.005986 \\
\hline $\mathrm{C} 4$ & -0.263064 & -0.658105 & -0.004524 \\
\hline $\mathrm{C} 5$ & 0.263064 & 0.658105 & -0.004524 \\
\hline C6 & 1.673671 & 0.710019 & -0.005982 \\
\hline S7 & 0.979581 & -1.875231 & -0.005985 \\
\hline $\mathrm{C} 8$ & 2.228417 & -0.605697 & -0.013925 \\
\hline $\mathrm{C} 9$ & 3.591363 & -0.818784 & -0.015196 \\
\hline $\mathrm{C} 10$ & 4.471985 & 0.292340 & -0.002476 \\
\hline $\mathrm{C} 11$ & 2.556554 & 1.816375 & 0.002691 \\
\hline $\mathrm{C} 12$ & 3.917634 & 1.603009 & 0.008557 \\
\hline H13 & 3.991490 & -1.825776 & 0.011389 \\
\hline $\mathrm{C} 14$ & 5.925635 & 0.088000 & 0.001607 \\
\hline H15 & 2.160276 & 2.826851 & -0.005847 \\
\hline H16 & 4.584778 & 2.456290 & -0.016394 \\
\hline $\mathrm{C} 17$ & -3.591363 & 0.818785 & -0.015196 \\
\hline $\mathrm{C} 18$ & -4.471985 & -0.292340 & -0.002475 \\
\hline C19 & -2.556553 & -1.816375 & 0.002692 \\
\hline $\mathrm{C} 20$ & -3.917634 & -1.603009 & 0.008557 \\
\hline $\mathrm{H} 21$ & -3.991490 & 1.825777 & 0.011388 \\
\hline $\mathrm{C} 22$ & -5.925635 & -0.088000 & 0.001607 \\
\hline $\mathrm{H} 23$ & -2.160276 & -2.826851 & -0.005846 \\
\hline $\mathrm{H} 24$ & -4.584778 & -2.456290 & -0.016393 \\
\hline $\mathrm{C} 25$ & 6.500226 & -1.049131 & -0.608357 \\
\hline $\mathrm{C} 26$ & 6.785554 & 1.024723 & 0.616593 \\
\hline $\mathrm{C} 27$ & 8.160505 & 0.825947 & 0.627522 \\
\hline $\mathrm{C} 28$ & 7.876726 & -1.236424 & -0.608271 \\
\hline $\mathrm{C} 29$ & 8.712214 & -0.302419 & 0.012610 \\
\hline $\mathrm{H} 30$ & 6.370407 & 1.888742 & 1.124374 \\
\hline H31 & 8.804801 & 1.546537 & 1.120550 \\
\hline H32 & 5.868141 & -1.765680 & -1.122539 \\
\hline H33 & 8.302204 & -2.106369 & -1.097767 \\
\hline H34 & 9.787028 & -0.452903 & 0.016900 \\
\hline
\end{tabular}

\section{TD-DFT Output}

Excitation energies and oscillator strengths were calculated with the $6-311+\mathrm{G}(\mathrm{d}, \mathrm{p})$ basis set, ${ }^{\mathrm{S}}$ using B3LYP method. ${ }^{\text {S2 }}$ All the calculations were performed on Gaussian 09 program.

3TTN

HOMO: 90, LUMO: 91

Excitation energies and oscillator strengths:

Excited State 1: $\quad$ Singlet-A $3.5330 \mathrm{eV}$

$350.93 \mathrm{~nm} \quad \mathrm{f}=0.1386<\mathrm{S} * * 2>=0.000$

$$
\begin{array}{lr}
89->93 & -0.19000 \\
90->91 & 0.67751
\end{array}
$$

This state for optimization and/or second-order correction. Copying the excited state density for this state as the 1-particle RhoCI density. 


\begin{tabular}{|c|c|c|}
\hline Excited State & Singlet-A & $3.5804 \mathrm{eV}$ \\
\hline $346.28 \mathrm{~nm} \quad \mathrm{f}=0.0773$ & $<\mathrm{S} * * 2>=0.000$ & \\
\hline $89->91$ & 0.61402 & \\
\hline $90->93$ & 0.34362 & \\
\hline Excited State & Singlet-A & $3.8807 \mathrm{eV}$ \\
\hline $319.49 \mathrm{~nm} \quad \mathrm{f}=0.0012$ & $<\mathrm{S} * * 2>=0.000$ & \\
\hline $88->91$ & -0.35202 & \\
\hline $90->92$ & 0.61094 & \\
\hline Excited State & Singlet-A & $4.0431 \mathrm{eV}$ \\
\hline $306.66 \mathrm{~nm} \quad \mathrm{f}=0.0000$ & $<\mathrm{S} * * 2>=0.000$ & \\
\hline $88->93$ & 0.16584 & \\
\hline $89->92$ & 0.68147 & \\
\hline Excited State & Singlet-A & $4.1498 \mathrm{eV}$ \\
\hline $298.77 \mathrm{~nm} \quad \mathrm{f}=0.0157$ & $<\mathrm{S} * * 2>=0.000$ & \\
\hline $88->91$ & 0.60548 & \\
\hline $90->92$ & 0.34742 & \\
\hline Excited State & Singlet-A & $4.3079 \mathrm{eV}$ \\
\hline $287.81 \mathrm{~nm} \quad \mathrm{f}=0.6474$ & $<\mathrm{S} * * 2>=0.000$ & \\
\hline $89->91$ & -0.33241 & \\
\hline $90->93$ & 0.60240 & \\
\hline Excited State & Singlet-A & $4.3759 \mathrm{eV}$ \\
\hline $283.33 \mathrm{~nm} \quad \mathrm{f}=0.0132$ & $<\mathrm{S} * * 2>=0.000$ & \\
\hline $88->92$ & 0.50147 & \\
\hline $89->93$ & -0.47568 & \\
\hline $90->91$ & -0.10612 & \\
\hline Excited State & Singlet-A & $4.4672 \mathrm{eV}$ \\
\hline $277.54 \mathrm{~nm} \quad \mathrm{f}=0.0000$ & $<\mathrm{S} * * 2>=0.000$ & \\
\hline $88->93$ & -0.10684 & \\
\hline $88->95$ & 0.16516 & \\
\hline $90->94$ & 0.67160 & \\
\hline Excited State & Singlet-A & $4.6458 \mathrm{eV}$ \\
\hline $266.87 \mathrm{~nm} \quad \mathrm{f}=0.0339$ & $<\mathrm{S} * * 2>=0.000$ & \\
\hline $88->94$ & 0.28048 & \\
\hline $90->95$ & 0.62873 & \\
\hline Excited State 10: & Singlet-A & $4.6723 \mathrm{eV}$ \\
\hline $265.36 \mathrm{~nm} \quad \mathrm{f}=0.5301$ & $<\mathrm{S} * * 2>=0.000$ & \\
\hline $87->91$ & -0.15578 & \\
\hline $88->92$ & 0.47694 & \\
\hline $89->93$ & 0.43778 & \\
\hline $89->95$ & -0.15573 & \\
\hline $90->91$ & 0.13850 & \\
\hline Excited State 11: & Singlet-A & $4.6738 \mathrm{eV}$ \\
\hline $265.27 \mathrm{~nm} \quad \mathrm{f}=0.0000$ & $<\mathrm{S} * * 2>=0.000$ & \\
\hline $86->91$ & 0.14922 & \\
\hline $88->93$ & 0.65672 & \\
\hline $89->92$ & -0.15039 & \\
\hline $90->94$ & 0.11142 & \\
\hline Excited State 12: & Singlet-A & $4.6758 \mathrm{eV}$ \\
\hline $265.16 \mathrm{~nm} \quad \mathrm{f}=0.0004$ & $<\mathrm{S} * * 2>=0.000$ & \\
\hline $89->94$ & 0.69139 & \\
\hline Excited State 13: & Singlet-A & $4.7832 \mathrm{eV}$ \\
\hline $259.21 \mathrm{~nm} \quad \mathrm{f}=0.0047$ & $<\mathrm{S} * * 2>=0.000$ & \\
\hline $87->91$ & 0.66797 & \\
\hline $89->93$ & 0.10315 & \\
\hline $89->95$ & -0.10503 & \\
\hline
\end{tabular}

$\begin{array}{ccc}\text { Excited State 14: } & \text { Singlet-A } & 4.8910 \mathrm{eV} \\ 253.49 \mathrm{~nm} \mathrm{f}=0.0689 & <\mathrm{S} * * 2>=0.000 & \\ 86->94 & 0.12260 \\ 89->93 & 0.14479 \\ 89->95 & 0.65400 & \end{array}$

Excited State 15: Singlet-A $4.9924 \mathrm{eV}$

$248.34 \mathrm{~nm} \quad \mathrm{f}=0.0031 \quad<\mathrm{S} * * 2>=0.000$

$84->91 \quad-0.18974$

$86->92 \quad 0.10900$

$88->94 \quad 0.11214$

$90->95 \quad-0.12259$

$90->96 \quad 0.64539$

Excited State 16: $\quad$ Singlet-A $\quad 5.0203 \mathrm{eV}$

$246.97 \mathrm{~nm} \quad \mathrm{f}=0.0000 \quad<\mathrm{S} * * 2>=0.000$

$86>91 \quad 0.60850$

$88->93 \quad-0.11245$

$88->96 \quad 0.12423$

$90->97 \quad-0.17387$

$90->99 \quad 0.22500$

Excited State 17: $\quad$ Singlet-A $\quad 5.0770 \mathrm{eV}$

$244.21 \mathrm{~nm} \quad \mathrm{f}=0.0000 \quad<\mathrm{S} * * 2>=0.000$

$$
\begin{array}{ll}
86->91 & 0.17549 \\
90->97 & 0.66929
\end{array}
$$

Excited State 18: $\quad$ Singlet-A $\quad 5.1743 \mathrm{eV}$

$239.62 \mathrm{~nm} \quad \mathrm{f}=0.0002 \quad<\mathrm{S} * * 2>=0.000$

$$
87->92 \quad 0.68375
$$

Excited State 19: $\quad$ Singlet-A $\quad 5.1743 \mathrm{eV}$

$239.62 \mathrm{~nm} \quad \mathrm{f}=0.0057 \quad<\mathrm{S} * * 2>=0.000$

$$
\begin{array}{lr}
87->93 & 0.12389 \\
87->95 & -0.13753 \\
88->94 & 0.58288 \\
90->95 & -0.26415 \\
90->96 & -0.16376
\end{array}
$$

Excited State 20: $\quad$ Singlet-A $\quad 5.1971 \mathrm{eV}$

$238.56 \mathrm{~nm} \quad \mathrm{f}=0.0028<\mathrm{S} * * 2>=0.000$

$$
90->98 \quad 0.69484
$$

\begin{tabular}{|c|c|c|}
\hline Excited State & Singlet-A & $3.6252 \mathrm{eV}$ \\
\hline $342.01 \mathrm{~nm} \quad \mathrm{f}=0.1446$ & $<\mathrm{S} * * 2>=0.000$ & \\
\hline $89->92$ & 0.34006 & \\
\hline $90->91$ & 0.61217 & \\
\hline Excited State & Singlet-A & $4.0870 \mathrm{eV}$ \\
\hline $303.37 \mathrm{~nm} \quad \mathrm{f}=0.6509$ & $<\mathrm{S} * * 2>=0.000$ & \\
\hline $87->91$ & -0.11037 & \\
\hline $89->91$ & 0.41685 & \\
\hline $90->92$ & 0.54952 & \\
\hline
\end{tabular}

2TTN

HOMO: 90, LUMO: 91

Excitation energies and oscillator strengths:

$$
\begin{array}{ccc}
\text { Excited State 1: } & \text { Singlet-A } & 3.4628 \mathrm{eV} \\
358.05 \mathrm{~nm} \mathrm{f}=0.0185 & <\mathrm{S} * * 2>=0.000 & \\
89->91 & 0.55908 \\
90->92 & -0.42690
\end{array}
$$

This state for optimization and/or second-order correction.

Copying the excited state density for this state as the 1-particle RhoCI density. 


\begin{tabular}{|c|c|c|c|c|c|}
\hline Excited State & Singlet-A & $4.1118 \mathrm{eV}$ & 89 -> 96 & 0.69373 & \\
\hline $301.53 \mathrm{~nm} \quad \mathrm{f}=0.2312$ & $<\mathrm{S} * * 2>=0.000$ & & Excited State 14: & Singlet-A & $4.8645 \mathrm{eV}$ \\
\hline $89->92$ & 0.61230 & & $254.87 \mathrm{~nm} \quad \mathrm{f}=0.1395$ & $<\mathrm{S} * * 2>=0.000$ & \\
\hline $90->91$ & -0.32838 & & $87->92$ & 0.68270 & \\
\hline Excited State & Singlet-A & $4.3598 \mathrm{eV}$ & 89 -> 99 & 0.14219 & \\
\hline $284.38 \mathrm{~nm} \quad \mathrm{f}=0.0000$ & $<\mathrm{S} * * 2>=0.000$ & & Excited State 15: & Singlet-A & $4.9008 \mathrm{eV}$ \\
\hline $88->91$ & 0.69337 & & $252.99 \mathrm{~nm} \quad \mathrm{f}=0.0000$ & $<\mathrm{S} * * 2>=0.000$ & \\
\hline Excited State & Singlet-A & $4.4456 \mathrm{eV}$ & $88->92$ & -0.12178 & \\
\hline $278.89 \mathrm{~nm} \quad \mathrm{f}=0.1064$ & $<\mathrm{S} * * 2>=0.000$ & & $89->94$ & 0.52523 & \\
\hline $87->91$ & 0.69278 & & $90->95$ & -0.43935 & \\
\hline Excited State & Singlet-A & $4.5421 \mathrm{eV}$ & Excited State 16: & Singlet-A & $4.9234 \mathrm{eV}$ \\
\hline $272.96 \mathrm{~nm} \quad \mathrm{f}=0.0000$ & $<\mathrm{S} * * 2>=0.000$ & & $251.83 \mathrm{~nm} \quad \mathrm{f}=0.0000$ & $<\mathrm{S} * * 2>=0.000$ & \\
\hline $86->91$ & 0.66342 & & $85->91$ & -0.14852 & \\
\hline $88->92$ & -0.22247 & & $86->92$ & -0.42915 & \\
\hline Excited State & Singlet-A & $4.6489 \mathrm{eV}$ & $89->95$ & 0.46148 & \\
\hline $266.70 \mathrm{~nm} \quad f=0.0000$ & $<\mathrm{S} * * 2>=0.000$ & & $90->94$ & -0.24906 & \\
\hline $90->93$ & 0.69528 & & Excited State 17: & Singlet-A & $4.9525 \mathrm{eV}$ \\
\hline Excited State & Singlet-A & $4.7133 \mathrm{eV}$ & $250.35 \mathrm{~nm} \quad \mathrm{f}=0.0000$ & $<\mathrm{S} * * 2>=0.000$ & \\
\hline $263.05 \mathrm{~nm} \quad \mathrm{f}=0.0000$ & $<\mathrm{S} * * 2>=0.000$ & & $86->91$ & 0.10408 & \\
\hline $89->93$ & 0.69621 & & $88->92$ & 0.16004 & \\
\hline Excited State 10: & Singlet-A & $4.7490 \mathrm{eV}$ & $89->94$ & 0.44985 & \\
\hline $261.08 \mathrm{~nm} \quad \mathrm{f}=0.0000$ & $<\mathrm{S} * * 2>=0.000$ & & $89->101$ & -0.11593 & \\
\hline $86->91$ & 0.19582 & & $90->95$ & 0.48274 & \\
\hline $88->92$ & 0.62716 & & Excited State 18: & Singlet-A & $5.1036 \mathrm{eV}$ \\
\hline $90->95$ & -0.23571 & & $242.93 \mathrm{~nm} \quad \mathrm{f}=0.0000$ & $<\mathrm{S} * * 2>=0.000$ & \\
\hline Excited State 11: & Singlet-A & $4.7730 \mathrm{eV}$ & $85->91$ & 0.28062 & \\
\hline $259.76 \mathrm{~nm} \quad \mathrm{f}=0.0000$ & $<\mathrm{S} * * 2>=0.000$ & & $86->92$ & 0.40213 & \\
\hline $88->93$ & -0.10120 & & $89->95$ & 0.49324 & \\
\hline $90->96$ & 0.69038 & & Excited State 19: & Singlet-A & $5.1611 \mathrm{eV}$ \\
\hline Excited State 12: & Singlet-A & $4.8329 \mathrm{eV}$ & $240.23 \mathrm{~nm} \quad \mathrm{f}=0.0032$ & $<\mathrm{S} * * 2>=0.000$ & \\
\hline $256.54 \mathrm{~nm} \quad \mathrm{f}=0.0000$ & $<\mathrm{S} * * 2>=0.000$ & & $90->97$ & 0.68998 & \\
\hline $85->91$ & 0.11115 & & $90->103$ & -0.10664 & \\
\hline $86->92$ & -0.31063 & & Excited State 20: & Singlet-A & $5.2561 \mathrm{eV}$ \\
\hline $90->94$ & 0.61330 & & $235.89 \mathrm{~nm} \quad \mathrm{f}=0.0000$ & $<\mathrm{S} * * 2>=0.000$ & \\
\hline Excited State 13: & Singlet-A & $4.8412 \mathrm{eV}$ & $89->97$ & 0.68109 & \\
\hline $256.10 \mathrm{~nm} \quad \mathrm{f}=0.0000$ & $<\mathrm{S} * * 2>=0.000$ & & $89->103$ & -0.14838 & \\
\hline
\end{tabular}

\section{References}

S1. (a) Hehre, W. J.; Ditchfield, R.; Pople, J. A. J. Chem. Phys. 1972, 56, 2257-2261. (b) Hariharan, P. C.; Pople, J. A. Theor. Chim. Acta. 1973, 28, 213-222. (c) Hariharan, P. C.; Pople, J. A. Mol. Phys. 1974, 27, 209-214. (d) Gordon, M. S. Chem. Phys. Lett. 1980, 76, 163-168. (e) Francl, M. M.; Pietro, W. J.; Hehre, W. J.; Binkley, J. S.; Defrees, D. J.; Pople, J. A.; Gordon, M. S. J. Chem. Phys. 1982, 77, 3654-3665. (f) Binning, R. C., Jr.; Curtiss, L. A. J. Comput. Chem. 1990, 11, 1206-1216. (g) Blaudeau, J. P.; McGrath, M. P.; Curtiss, L. A.; Radom, L. J. Chem. Phys. 1997, 107, 5016-5021. (h) Rassolov, V. A.; Pople, J. A.; Ratner, M. A.; Windus, T. L. J. Chem. Phys. 1998, 109, 1223-1229. (i) Rassolov, V. A.; Ratner, M. A.; Pople, J. A.; Redfern, P. C.; Curtiss, L. A. J. Comput. Chem. 2001, 22, 976-984.

S2. (a) Becke, A. D. J. Chem. Phys. 1993, 98, 5648-5652. (b) Lee, C.; Yang, W.; Parr, R. G. Phys. Rev. B 1988, 37, 785-789. (c) Miehlich, B.; Savin, A.; Stoll, H.; Preuss, H. Chem. Phys. Lett. 1989, 157, 200-206.

S3. (a) Wachters, A. J. H. J. Chem. Phys. 1970, 52, 1033-1036. (b) Hay, P. J. J. Chem. Phys. 1977, 66, 4377-4384. (c) Raghavachari, K.; Trucks, G. W. J. Chem. Phys. 1989, 91, 1062-1065.

(End) 